\title{
ANTOINE BOMMIER
}

\section{Prolongement méromorphe de la matrice de diffusion pour les problèmes à $N$ corps à longue portée}

Mémoires de la S. M. F. $2^{e}$ série, tome 59 (1994)

<http://www.numdam.org/item?id=MSMF_1994_2_59_1_0>

CC Mémoires de la S. M. F., 1994, tous droits réservés.

L'accès aux archives de la revue « Mémoires de la S. M. F. » (http://smf. emath.fr/Publications/Memoires/Presentation.html) implique l'accord avec les conditions générales d'utilisation (http://www.numdam.org/conditions). Toute utilisation commerciale ou impression systématique est constitutive d'une infraction pénale. Toute copie ou impression de ce fichier doit contenir la présente mention de copyright.

\section{Numdam}


Société Mathématique de France

Mémoire 59

Supplément au Bulletin de la S.M.F.

Tome 122, 1994, fascicule 4.

\section{Prolongement méromorphe de la matrice de diffusion pour les problèmes à $\mathbf{N}$ corps à longue portée

\author{
Antoine BOMMIER
}

\section{Résumé}

Nous étudions certaines propriétés des matrices de diffusion 2 amas $k$ amas pour les problèmes à $\mathrm{N}$ corps à longue portée. Nous montrons en particulier, avec des hypothèses d'analyticité sur les potentiels, que l'on peut les prolonger méromorphiquement à des énergies complexes, et que leurs noyaux sont analytiques.

\section{Abstract}

We study some analytic properties of the 2 clusters $k$ clusters Scattering Matrices for N-Body problems with long-range interactions. In particular we prove, with some hypothesis on the analyticity of the potentials, the existence of their meromorphic continuations and the analyticity of their kernels.

Classification A.M.S. (1985). 35A15, 35B37, 35J10, 35P25, 35S15, 49E15.

Texte reçu le 16 novembre 1993

Ecole Polytechnique, Centre de Mathématiques (CMAT), U.R.A. 169

91128 Palaiseau Cedex, France. 



\section{Table des matières}

1. Introduction $\mathbf{5}$

1.1 Notations . . . . . . . . . . . . . . . 7

1.1.1 Notations générales. . . . . . . . . . . . 7

1.1.2 Notations liées au problème à $N$ corps. . . . . . . 8

1.2 Hypothèses . . . . . . . . . . . . . . . . . . . 10

1.3 La construction d'Isozaki-Kitada. . . . . . . . . . . . . . 11

1.4 Définition des opérateurs d'onde et de la matrice de diffusion. 13

1.5 Résultats : . . . . . . . . . . . . 17

2. Une nouvelle construction des opérateurs d'onde. 19

2.1 Construction des opérateurs d'onde pour un canal de réaction à deux amas. . . . . . . . . . . . . . 20

2.2 Démonstration de la proposition $2.4 \ldots \ldots$. . . . . . 24

2.3 Construction des opérateurs d'onde pour un canal de réaction quelconque. ................. 36

3. Démonstration du théorème $1.9 \quad 39$

3.1 Prolongement de $M_{1} \ldots \ldots \ldots \ldots$. . . . . . . . . . . . . . . . . . 42

3.2 Prolongement de $M_{2} \ldots \ldots \ldots \ldots$. . . . . . . 47

4. Démonstration du théorème $1.10 \quad 55$

4.1 Démonstration de $(2) \ldots \ldots \ldots \ldots 6$

4.2 Démonstration de $(1) \ldots \ldots \ldots \ldots$

A. Appendice : Les "boost-distorsions" et le problème à $\mathrm{N}$ corps.

Bibliographie 



\section{Introduction}

Afin d'expliquer les résultats des expériences de diffusion (de collision atomique par exemple), les physiciens utilisent fréquemment des modèles faisant intervenir les valeurs de la matrice de diffusion $S(\lambda)$ à des énergies complexes. Tel est le cas en particulier pour l'interprétation des pics qu'ils observent sur les spectres d'absorptions, encore appelés résonances, qui peuvent s'associer assez naturellement aux pôles complexes de $S(\lambda)$

D'un point de vue mathématique cela ne va pas sans poser quelques interrogations, étant donné qu'il est loin d'être évident a priori que l'on puisse définir correctement $S(\lambda)$ pour des énergies $\lambda$ complexes. Plusieurs travaux ont tenté de répondre à cette question en montrant pour certains problèmes que $S(\lambda)$ peut se prolonger méromorphiquement à des énergies complexes, et que de plus ses pôles s'identifient alors à ceux de la résolvante. (Faisant ainsi le lien avec la définition des résonances plus généralement utilisée en Mathématiques.) Le cas du problème à deux corps avec des potentiels à courte portée était en particulier traité assez complètement au cours des années 1970. (Voir par exemple [Sh-Th], [Ba1] et [Ba2])

Pour ce qui est des problèmes à longue portée, la question n'a été résolue que plus tardivement, en 1985, par Gérard et Martinez [G-M] qui montraient l'existence d'un prolongement méromorphe et l'identification des pôles de ce prolongement à ceux de la résolvante, complétant ainsi l'étude du problème à deux corps.

En revanche en ce qui concerne le problème à $\mathrm{N}$ corps les résultats à ce sujet se limitaient aux potentiels à courte portée et restaient qui plus est 
A. BOMMIER

très partiels. Parmi les plus significatifs on trouve principalement ceux de Balslev (1980) [Ba3], qui ne traitaient que de la diffusion élastique 2 amas - 2 amas, sous le seuil d'énergie des décompositions à trois amas, prouvant dans ce cas l'existence d'un prolongement méromorphe de la matrice de diffusion, prolongement dont les pôles sont des pôles de la résolvante.

Dans cet article nous traiterons des problèmes à $N$ corps, avec des potentiels à longue portée. Nous montrerons que les matrices de diffusion 2 amas - $k$ amas, ( pour $2 \leq k \leq N$ ), se prolongent méromorphiquement et que leurs pôles sont aussi des pôles de la résolvante. Nous prouverons en sus que leurs noyaux sont analytiques par rapport aux variables angulaires en dehors des plans de collision, et en dehors de la diagonale dans le cas d'une collision élastique.

Les méthodes que nous utiliserons sont radicalement différentes de celles de [Ba3], et s'approchent beaucoup plus de celles utilisées pour l'étude du problème à deux corps dans [G-M]. En particulier nous utiliserons une définition stationnaire des opérateurs d'onde introduite par Isozaki et Kitada dans $[\mathrm{I}-\mathrm{K}]$.

Dans la suite de l'introduction nous précisons tout d'abord les hypothèses, nos notations, et la définition des opérateurs d'onde utilisée. On rappellera brièvement en particulier la construction originale d'Isozaki-Kitada. Nous y donnerons enfin l'énoncé de nos résultats (Théorèmes 1.9 et 1.10).

La démonstration nécessitera dans un premier temps la formulation d'une nouvelle construction des opérateurs d'onde, qui tout en redonnant la définition proposée en introduction, aura de "bonnes" propriétés, indispensables à la suite du raisonnement.

Utilisant cette construction nous effectuerons alors les démonstrations de nos résultats.

L'appendice est un bref développement de la théorie des "boost-distorsions" pour le problème à $N$ corps, qui est un élément important de la démonstration. Nous l'avons mis à part car ne traitant pas spécifiquement de la diffusion il peut être utilisé dans un cadre différent. 


\section{$1.1 \quad$ Notations}

\subsubsection{Notations générales.}

Commençons par préciser quelques notations relatives aux opérateurs linéaires. Pour tout espace de Hilbert $\mathcal{H}$ nous noterons $B(\mathcal{H})$ l'ensemble des opérateurs linéaires bornés de $\mathcal{H}$ dans lui même. Pour tout opérateur $T$ linéaire fermé sur $\mathcal{H}$, borné ou non, nous définirons $\sigma(T)$ le spectre de $T$, $\rho(T)$ l'ensemble résolvant de $T, \sigma_{e}(T)$ le spectre essentiel de $T$ et $\sigma_{d}(T)$ le spectre discret de $T$.

Pour deux opérateurs linéaires $A$ et $B$ nous utiliserons la notation $a d_{A}(B)$ pour le commutateur $[A, B]=A B-B A$. Si $A$ est auto-adjoint nous noterons $\langle A\rangle=\left(1+A^{2}\right)^{\frac{1}{2}}$.

La théorie des dilatations analytiques nous sera fort utile dans cet article. Pour $\theta \in \mathbb{R}$ assez petit on définit l'opérateur unitaire $U_{\theta}$ par :

$$
U_{\theta}:\left\{\begin{aligned}
L^{2}\left(\mathbb{R}^{n}\right) & \rightarrow L^{2}\left(\mathbb{R}^{n}\right) \\
f(x) & \rightarrow(1+\theta)^{n / 2} f(x(1+\theta))
\end{aligned}\right.
$$

Pour $\mu>0$ on définit l'ensemble des vecteurs de $L^{2}\left(\mathbb{R}^{n}\right), \mu$ - analytiquement dilatables, que l'on notera $L^{2}\left(\mathbb{R}^{n}\right)_{\mu}$, comme étant l'ensemble des vecteurs $\psi \in L^{2}\left(\mathbb{R}^{n}\right)$ tels que $U_{\theta} \psi$ se prolonge holomorphiquement dans une région $|\operatorname{Im}(\theta)|<\mu$ et tels que :

$$
\exists C \in \mathbb{R}|| \operatorname{Im}(\theta) \mid<\mu \Rightarrow\left\|U_{\theta} \psi\right\|_{L^{2}\left(\mathbb{R}^{n}\right)} \leq C
$$

On définit alors la norme $\|\psi\|_{L^{2}\left(\mathbb{R}^{n}\right)_{\mu}}$ par la meilleure constante $C$ que l'on peut prendre dans l'inégalité ci-dessus.

De même on définit l'ensemble des opérateurs bornés $\mu$ - analytiquement dilatables, que l'on notera $B_{\mu}\left(L^{2}\left(\mathbb{R}^{n}\right)\right)$, comme étant l'ensemble des opérateurs $M \in B\left(L^{2}\left(\mathbb{R}^{n}\right)\right)$ tels que $U_{\theta} M U_{\theta}^{-1}$ se prolonge holomorphiquement dans une région $|\operatorname{Im}(\theta)|<\mu$ et tels que :

$$
\exists C \in \mathbb{R}|| \operatorname{Im}(\theta) \mid<\mu \Rightarrow\left\|U_{\theta} M U_{\theta}^{-1}\right\| \leq C
$$

On définit là aussi la norme $\|M\|_{B_{\mu}\left(L^{2}\left(R^{n}\right)\right)}$ par la meilleure constante $C$ que l'on peut prendre dans cette inégalité. Il est clair que $B_{\mu}\left(L^{2}\left(\mathbb{R}^{n}\right)\right)$ a une structure d'algèbre.

Nous rappelons la définition des classes de fonctions Gevrey sur la sphère 
unité $S$ : nous dirons qu'une fonction $f$ est Gevrey $s$ sur $S$ si il existe une constante $C$ telle que pour tout entier $k$ on ait :

$$
\left\|D^{k} f\right\| \leq C^{k}(k !)^{s}
$$

$D^{k} f$ étant la différentielle $k^{i e m e}$ de $f$. Nous écrirons $f \in G^{s}(S)$

Pour $x, y \in \mathbb{C}^{n}$ nous utiliserons la notation régularisante $\langle x\rangle=\left(1+|x|^{2}\right)^{\frac{1}{2}}$ et nous définirons la fonction cosinus par $\cos (x, y)=\cos (\operatorname{Re}(x), \operatorname{Re}(y))$.

L'écriture $f(x)=O\left(\langle x\rangle^{-\infty}\right)$ signifiera que $\langle x\rangle^{p}\|f(x)\|$ est borné pour tout $p \in \mathbb{N}$.

Enfin pour une fonction réelle $g(t)$ donnée, nous utiliserons pour simplifier, quand cela ne porte pas à confusion, la notation générique $\chi(g(t) \geq C)$ qui se définit rigoureusement par :

$$
\chi(g(t) \geq C)=\chi_{0}(g(t)-C)
$$

où $\chi_{0}$ est une fonction de troncature de classe $C^{\infty}$ telle que :

$$
\left\{\begin{array}{l}
\chi_{0}(x)=1 \text { pour } x \geq 0 \\
\chi_{0}(x)=0 \text { pour } x \leq-\epsilon
\end{array}\right.
$$

avec $0<\epsilon<<1$.

La notation $\chi(g(t) \leq C)$ se définit de façon tout à fait similaire.

\subsubsection{Notations liées au problème à $N$ corps.}

Dans l'étude du Hamiltonien à $N$ corps (1.5) nous séparons dans un premier temps, de façon tout à fait classique, le mouvement du centre de masse, ce qui nous ramène à l' étude du Hamiltonien $H=-\Delta_{\left.\right|_{X}}+V(x)$ opérant sur $L^{2}(X)$ où $X$ est l'espace :

$$
X=\left\{x \in \mathbb{R}^{n N} \mid \sum m_{i} x_{i}=0\right\}
$$

et $\Delta_{\left.\right|_{X}}$ l'opérateur de Laplace-Beltrami associé à la métrique :

$$
q(x, y)=\sum 2 m_{i}\left(x_{i} . y_{i}\right)
$$

Nous appellerons décomposition en $k$ amas, toute partition $\left(A_{1}, \cdots A_{k}\right)$, de $\{1, \cdots, N\}$. L'ensemble des décompositions est muni d'une relation d'ordre définie par : $a \subset b$ si $a$ est une partition plus fine que $b$. 
Nous noterons conventionnellement $(i, j)$ la partition à $N-1$ amas dont un est formé par la paire $(i, j)$. Ainsi la relation $(i, j) \subset a$ sera vérifiée si et seulement si les particules $i$ et $j$ appartiennent à un même amas $A_{k}$ de la décomposition $a$.

A chaque décomposition $a$ nous faisons correspondre deux sous espaces $X_{a}$ et $X^{a}$ définis par :

$$
\begin{gathered}
X_{a}=\left\{x \in X \mid x_{i}=x_{j} \text { si }(i, j) \subset a\right\} \\
X^{a}=\left\{x \in X \mid \sum_{i \in A_{j}} m_{i} x_{i}=0\right\}
\end{gathered}
$$

$X^{a}$ représente l'espace des variables internes aux amas, alors que $X_{a}$ représente l'espace des variables externes, variables décrivant la position relative des centres de masse de chacun des amas.

Ces deux sous espaces sont orthogonaux pour la métrique $q(x, y)$, et l'on a

$$
X=X_{a} \oplus X^{a}
$$

Nous noterons $S_{a}$ la sphère unité de $X_{a}$.

On pose pour toute décomposition $a$ :

$$
I_{a}(x)=\sum_{(i, j) \not \subset a} V_{i j}(x), V^{a}\left(x^{a}\right)=\sum_{(i, j) \subset a} V_{i j}(x) \text { et } H_{a}=H-I_{a} .
$$

On a :

$$
H_{a}=I_{\left.\right|_{X^{a}}} \otimes\left(-\Delta_{\left.\right|_{X_{a}}}\right)+\left(-\Delta_{\left.\right|_{X^{a}}}+V^{a}\left(x^{a}\right)\right) \otimes I_{\left.\right|_{X_{a}}}
$$

ce que l'on notera plus couramment $H_{a}=-\Delta_{\left.\right|_{X_{a}}}+H^{a}, H^{a}$ étant le Hamiltonien interne $-\Delta_{\left.\right|_{X^{a}}}+V^{a}\left(x^{a}\right)$ opérant sur $L^{2}\left(X^{a}\right)$.

Nous noterons $\sigma^{a}=\sigma_{d}\left(H^{a}\right)$ et $\tau_{a}=\underset{b \subset a, b \neq a}{\bigcup} \sigma^{b} \cup\{0\}$ le spectre purement ponctuel et l'ensemble des seuils du Hamiltonien interne $H^{a}$.

Les notations $\sigma(H)$ et $\tau(H)$, qui sont les valeurs propres et les seuils du Hamiltonien $H$, correspondent à celles définies ci-dessus dans le cas trivial où $a$ est la décomposition en un amas.

On appelle canal de réaction $\alpha=\left(a, \psi_{\alpha}, \epsilon_{\alpha}\right)$ la donnée d'une décomposition $a$, et d'un vecteur propre, $\psi_{\alpha}$, de $H^{a}$, de valeur propre $\epsilon_{\alpha}$.

Pour un intervalle d' énergie $\Delta$ et un canal de réaction donnés nous noterons $\pi_{\alpha}$ la projection orthogonale sur le vecteur $\psi_{\alpha}$, et $p_{\alpha}$ l'application définie par :

$$
p_{\alpha}:\left\{\begin{aligned}
L^{2}\left(X_{a}\right) & \rightarrow L^{2}(X) \\
\phi & \rightarrow E_{\Delta}\left(H_{a}\right) \phi \otimes \psi_{\alpha}
\end{aligned}\right.
$$


Nous rappelons que sous les hypothèses $(H)$ que nous considérerons, et sous la condition $\epsilon_{\alpha} \notin \tau_{a}, \psi_{\alpha}$ est à décroissance exponentielle en $x^{a}$ (voir l'article $[\mathrm{F}-\mathrm{H}])$.

Pour chaque canal de réaction $\alpha$ on définit une transformée de Fourier associée, $\mathcal{F}_{\alpha}$, comme suit :

Soit $\mathcal{F}_{\alpha}(\lambda) \in B\left(L_{s}^{2}\left(X_{a}\right), L^{2}\left(S_{a}\right)\right), s>\frac{1}{2}, \lambda>\epsilon_{\alpha}$ défini par :

$$
\left(\mathcal{F}_{\alpha}(\lambda) f\right)(\omega)=\left(2\left(\lambda-\epsilon_{\alpha}\right)\right)^{(n-2) / 4}\left(\mathcal{F} f\left(\sqrt{2\left(\lambda-\epsilon_{\alpha}\right)} \omega\right)\right.
$$

où $\mathcal{F} f$ est la transformée de Fourier de $f$, c'est à dire :

$$
\mathcal{F} f(\xi)=\hat{f}(\xi)=(2 \pi)^{-n / 2} \int_{R^{n}} e^{-i x . \xi} f(x) d x
$$

On note $\hat{\mathcal{H}}$ l'espace de Hilbert $L^{2}\left(\left(\epsilon_{\alpha},+\infty\right) ; L^{2}\left(S_{a}^{n-1}\right)\right)$ et $\mathcal{F}_{\alpha} \in B\left(L^{2}\left(X_{a}\right) ; \hat{\mathcal{H}}\right)$ donné par :

$$
\left(\mathcal{F}_{\alpha} f\right)(\lambda, \omega)=\left(\mathcal{F}_{\alpha}(\lambda) f\right)(\omega) \quad \forall f \in L_{s}^{2}\left(X_{a}\right)
$$

$\mathcal{F}_{\alpha}$ se prolonge en un unique opérateur unitaire et $\forall f, g \in L_{s}^{2}\left(X_{a}\right)$ :

$$
(f, g)=\left(\mathcal{F}_{\alpha} f, \mathcal{F}_{\alpha} g\right)_{\hat{\mathcal{H}}}=\int_{\epsilon_{\alpha}}^{+\infty}\left(\mathcal{F}_{\alpha}(\lambda) f, \mathcal{F}_{\alpha}(\lambda) g\right)_{L^{2}\left(S_{a}^{n-1}\right)} d \lambda
$$

D'autre part pour toute fonction $k$ :

$$
\left(\mathcal{F}_{\alpha}\left(k\left(D_{x_{a}}^{2}+\epsilon_{\alpha}\right) f\right)\right)(\lambda, \omega)=k(\lambda) \mathcal{F}_{\alpha}(\lambda) f
$$

\subsection{Hypothèses}

Nous considérons le Hamiltonien à N corps :

$$
H=-\sum_{i=1}^{N} \frac{1}{2 m_{i}} \Delta_{x_{i}}+\sum_{1 \leq i \leq N} V_{i j}\left(x_{i}-x_{j}\right) \quad ; \quad x_{i} \in \mathbb{R}^{n}
$$

où les potentiels $V_{i j}$ sont supposés vérifier les hypothèses suivantes :

$$
(H)\left\{\begin{array}{l}
\text { Les potentiels } V_{i j} \text { se prolongent holomorphiquement } \\
\text { dans une région : } \\
\quad D_{\epsilon}=\left\{x \in \mathbb{C}^{n}|| \operatorname{Im}(x) \mid \leq \epsilon\langle\operatorname{Re}(x)\rangle\right\} \\
\text { avec } \epsilon>0 \text { et vérifient la condition de décroissance suivante : } \\
V_{i j}(x)=O\left(\langle x\rangle^{-\rho}\right) \quad \text { dans } D_{\epsilon} .
\end{array}\right.
$$

Nous ferons en outre quelquefois des hypothèses supplémentaires sur les canaux de réaction $\alpha=\left(a, \psi_{\alpha}, \epsilon_{\alpha}\right)$ (canal d'entrée) et $\beta=\left(b, \psi_{\beta}, \epsilon_{\beta}\right)$ (canal 
de sortie) ainsi que sur l'intervalle d'énergie $[d, M]$ que nous considérons. Nous les formulons ainsi :

$(E)$

1) $\epsilon_{\alpha}$ et $\epsilon_{\beta}$ sont des valeurs propres, respectivement de $H^{a}$ et $H^{b}$, simples et isolées.

2) $[d, M] \subset] \sup \left(\epsilon_{\alpha}, \epsilon_{\beta}\right),+\infty[$

3) $[d, M] \cap \tau(H)=\emptyset$

4) $a$ est une décomposition en deux amas.

5) $\beta$ vérifie au moins une des hypothèses suivantes :

5.1) $b$ est une décomposition en deux amas.

5.2) $\left.] \epsilon_{\beta}, d\right] \cap \tau(H)=\emptyset$

Commentaires:

- La condition $(E .2)$ signifie seulement que l'on se limite aux énergies où les canaux de réaction $\alpha$ et $\beta$ sont ouverts, et n'est rien d'autre que la condition physique pour qu'il y ait diffusion.

- La condition ( $E$.5.2) nous impose une contrainte en énergie supplémentaire lorsque l'on étudie la diffusion 2 amas $-k$ amas, $(k>2)$. Elle limite l'étude à un intervalle d'énergie compris entre $\epsilon_{\beta}$ et le premier seuil qui lui est supérieur. Il est intéressant de noter que pour la diffusion 2 amas$N$ amas cette hypothèse est redondante, étant une conséquence triviale de $(E$.2). On sait en effet que sous les hypothèses $(H)$ il n'y a pas de seuil à énergie positive.

\subsection{La construction d'Isozaki-Kitada.}

Comme nous l'avons précisé dans l'introduction nous utiliserons dans ce travail la construction des opérateurs d'onde d'Isozaki-Kitada [I-K], que nous rappelons ici brièvement.

Dans [I-K] Isozaki et Kitada s'intéressent au problème à deux corps avec un potentiel $V(x)$ vérifiant les hypothèses de régularité et de décroissante suivante :

$$
\exists \rho>0 \text { tel que } \forall \alpha \exists C_{\alpha} \quad|\quad| \partial^{\alpha} V(x) \mid \leq C_{\alpha}\langle x\rangle^{-\rho-|\alpha|}
$$

Leur démarche est la suivante. Dans un premier temps ils construisent deux solutions, $\phi^{ \pm}$de l'équation eikonale :

$$
\left(\nabla_{x} \phi^{ \pm}(x, \xi)\right)^{2}+V(x)=\xi^{2}
$$

supportée chacune dans des régions du type :

$$
\left\{|x|>R,|\xi|>d, \pm \cos (x, \xi) \geq \pm \sigma^{ \pm}\right\}
$$


et satisfaisant les estimations :

$$
\forall \alpha, \beta \in \mathbb{N}^{n}\left|\partial_{x}^{\alpha} \partial_{\xi}^{\beta}(\phi(x, \xi)-x . \xi)\right| \leq C_{\alpha \beta}\langle x\rangle^{1-\rho-|\alpha|}
$$

Ils recollent ces deux fonctions en posant :

$$
\phi(x, \xi)=\chi\left(\cos (x, \xi) \leq \sigma^{-}\right) \phi^{-}(x, \xi)+\chi\left(\cos (x, \xi) \geq \sigma^{+}\right) \phi^{+}(x, \xi)
$$

A l'aide de cette fonction de phase ils définissent le modificateur $J$ par :

$$
J f(x)=(2 \pi)^{-n} \iint e^{i(\phi(x, \xi)-y \cdot \xi)} f(y) d y d \xi
$$

et les opérateurs d'onde par :

$$
W^{ \pm}=s-\lim _{t \rightarrow \pm \infty} e^{i t H} J e^{-i t H_{0}} E_{\Delta}\left(H_{0}\right)
$$

\section{Commentaires:}

La construction des opérateurs d'onde généralement utilisés auparavant pour les problèmes à longue portée était celle de Dollard, se basant sur la construction d'un modificateur dépendant du temps et se référant ainsi directement à l'évolution classique. La construction d'Isozaki-Kitada trouve quant à elle son origine dans le cadre même de la mécanique quantique, étant inspirée en particulier par l'expression des fonctions d'onde généralisées. Les idées qui ont motivé ces deux types de construction sont donc de nature assez différente, mais offrent malgré cela des développements très similaires, faisant intervenir toutes deux la résolution d'une équation de mécanique classique, qui permet de décrire l'évolution asymptotique des états de diffusion (le modèle de l'évolution libre ne convenant plus pour les problèmes à longue portée). Il s'agit de l'équation de Hamilton-Jacobi pour la construction de Dollard et de l'équation eikonale pour celle de Isozaki-Kitada. En fin de compte on peut vérifier que ces deux types d'opérateurs coïncident rigoureusement.

La méthode d'Isozaki-Kitada offre en sus quelques avantages techniques qui ont permis d'établir des résultats tels que la régularité des amplitudes de diffusion ou leur prolongement méromorphe en énergie. La raison principale en est que l'on peut utiliser bien évidemment d'autres modificateurs dans la construction des opérateurs d'onde sans que cela change la limite (1.8). C'est cette idée qui est à la base des démarches de [I-K], [G-M], et aussi de cet article.

Notons enfin que pour le problème à courte portée, $(\rho>1)$, Wang a proposé dans [W] une construction améliorée des fonctions de phase $\phi^{ \pm}$. Celles-ci, tout en vérifiant les propriétés (1.6) et (1.7), ont pour avantage 
de redonner par (1.8) les opérateurs d'onde usuels, obtenus dans ce cas en prenant $J=1$. Comme nous n'utilisons par la suite que les propriétés (1.6) et (1.7) nous pouvons utiliser cette construction dès que $\rho>1$. Cela nous permet en particulier de faire le lien avec la théorie usuelle de la diffusion pour les problèmes à courte portée.

\subsection{Définition des opérateurs d'onde et de la matrice de diffusion.}

Nous avons choisi de généraliser de façon canonique la construction d'Isozaki-Kitada pour le problème à $\mathrm{N}$ corps. Précisons ce que nous entendons par là.

Les états de diffusion, encore appelés canaux de réaction, sont définis par la donnée d'une partition des $\mathrm{N}$ corps en $k$ sous ensembles, ce que l'on nomme décomposition en $k$ amas, et d'un état lié pour chacun des amas correspondants. La première idée physique qui vient à l'esprit quand on cherche à donner une approximation de l'évolution asymptotique de tels états consiste à dire que les amas s'éloignant les uns des autres, aux temps grands, chacun des amas voit approximativement les autres comme des particules ponctuelles. Formulé mathématiquement cela revient à dire que $I_{a}(x) \simeq I_{a}\left(x_{a}\right)$ le long de l'évolution, lorsque $t \rightarrow \pm \infty$.

Lorsque $a$ est une décomposition en deux amas $I_{a}\left(x_{a}\right)$ n'est alors rien de plus qu'un potentiel à deux corps, et l'on peut reprendre la construction de la fonction de phase d'Isozaki-Kitada sans modification. En revanche lorsque $a$ est une décomposition en $k$ amas, $(k>2), I_{a}\left(x_{a}\right)$ est un potentiel à $k$ corps et cela pose une réelle difficulté. En particulier $I_{a}\left(x_{a}\right) \nrightarrow 0$ quand $\left|x_{a}\right| \rightarrow \infty$. Nous éviterons cette difficulté en ne traitant la diffusion que dans les directions hors des plans de collision, c'est à dire en évitant les états où la distance entre deux des $k$ amas ne croit pas au moins proportionnellement à la taille totale du système.

Posons quelques définitions permettant de formuler cela :

Définition 1.1 - Pour toute décomposition a nous noterons :

$$
\begin{gathered}
Z_{a}=X_{a} \backslash \cup_{b \not \subset a} X_{b} \\
\tilde{S}_{a}=S_{a} \cap Z_{a}
\end{gathered}
$$

Nous n'étudierons la diffusion que pour des directions se trouvant dans $\tilde{S}_{a}$. Notons que dans le cas où $a$ est une décomposition en 2 amas $Z_{a}=X_{a} \backslash\{0\}$ et $\tilde{S}_{a}=S_{a}$. Nous ne ferons donc aucune restriction dans ce cas particulier. 
La définition qui suit ne sert qu'à préciser les propriétés de support des fonctions de troncature que nous serons amenés à faire intervenir pour traiter le cas \# $a>2$.

Définition 1.2 - Soit a une décomposition, telle que \#a>2.

Pour tout compact $K_{a} \subset \tilde{S}_{a}$ nous définissons les compacts $K_{a}(\epsilon),(\epsilon \in] 0,1[)$, par :

$$
K_{a}(\epsilon)=\left\{x \in \tilde{S}_{a} \mid \operatorname{dist}\left(x, K_{a}\right) \leq \epsilon \operatorname{dist}\left(K_{a}, S_{a} \backslash \tilde{S}_{a}\right\}\right.
$$

et nous définissons les fonctions de troncatures $\chi_{a_{0}}, \chi_{a_{1}}, \chi_{a_{2}}$ par :

$$
\begin{array}{llll}
\chi_{a_{0}}\left(\omega_{a}\right)=1 \quad \forall \omega_{a} \in K_{a} & \text { et } & \text { supp } \chi_{a_{0}} \subset K_{a}\left(\frac{1}{7}\right) \\
\chi_{a_{1}}\left(\omega_{a}\right)=1 \quad \forall \omega_{a} \in K_{a}\left(\frac{2}{7}\right) & \text { et } & \text { supp } \chi_{a_{1}} \subset K_{a}\left(\frac{3}{7}\right) \\
\chi_{a_{2}}\left(\omega_{a}\right)=1 \quad \forall \omega_{a} \in K_{a}\left(\frac{4}{7}\right) & \text { et } & \text { supp } \chi_{a_{2}} \subset K_{a}\left(\frac{5}{7}\right)
\end{array}
$$

Nous définissons enfin $0<\tilde{\sigma}_{K_{a}}<\sigma_{K_{a}}<1$ par:

$$
\sigma_{K_{a}}=\sup \left\{\cos \left(\omega, \omega^{\prime}\right) \mid \omega \in K_{a}\left(\frac{1}{7}\right) \quad, \quad \omega^{\prime} \in K_{a}\left(\frac{3}{7}\right) \backslash K_{a}\left(\frac{2}{7}\right)\right\}
$$

et

$$
\tilde{\sigma}_{K_{a}}=\sup \left\{\cos \left(\omega, \omega^{\prime}\right) \mid \omega \in K_{a}\left(\frac{1}{7}\right), \omega^{\prime} \in K_{a}\left(\frac{5}{7}\right) \backslash K_{a}\left(\frac{4}{7}\right)\right\}
$$

Pour ne pas avoir à distinguer à chaque fois les cas \# $a=2$ et \#a>2 nous adopterons les définitions suivantes :

Définition 1.3 - Pour toute décomposition a en deux amas nous noterons conventionnellement :

$$
\begin{gathered}
\left.K_{a}(\epsilon)=S_{a} \quad, \quad \forall \epsilon \in\right] 0,1[ \\
\chi_{a_{0}}\left(\omega_{a}\right)=\chi_{a_{1}}\left(\omega_{a}\right)=\chi_{a_{2}}\left(\omega_{a}\right)=1 \quad, \quad \forall \omega_{a} \in S_{a} \\
\sigma_{K_{a}}=-\frac{1}{2}
\end{gathered}
$$

Venons-en maintenant à la définition même des opérateurs d'onde, en suivant la démarche de Isozaki-Kitada. Nous commençons par définir :

Définition 1.4 - Pour toute décomposition a on pose :

$$
\tilde{I}_{a}(x)=I_{a}(x) \chi_{a_{2}}\left(\frac{x_{a}}{\left|x_{a}\right|}\right)
$$


Lemme 1.5 - Sous les hypothèses $(H)$, pour tout $d_{a}>0$, $-1<\sigma_{a}^{-}<\sigma_{a}^{+}<1$, il existe $R>0$ et $\phi_{a}^{ \pm}$des fonctions réelles, de classe $C^{\infty}$ sur :

$$
\Gamma_{a}^{ \pm}\left(R, d_{a}, \sigma_{a}^{ \pm}\right)=\left\{\left|x_{a}\right|>R,\left|\xi_{a}\right|>d_{a}, \pm \cos \left(x_{a}, \xi_{a}\right) \geq \pm \sigma_{a}^{ \pm}\right\}
$$

tels que:

$$
\begin{gathered}
\left(\nabla_{x_{a}} \phi_{a}^{ \pm}\left(x_{a}, \xi_{a}\right)\right)^{2}+\tilde{I}_{a}\left(x_{a}\right)=\xi_{a}^{2} \\
\forall \alpha, \beta \in \mathbb{N}^{n}\left|\partial_{x_{a}}^{\alpha} \partial_{\xi_{a}}^{\beta}\left(\phi\left(x_{a}, \xi_{a}\right)-x_{a} \cdot \xi_{a}\right)\right| \leq C_{\alpha \beta}\left\langle x_{a}\right\rangle^{1-\rho-|\alpha|}
\end{gathered}
$$

Démonstration: Il suffit de remarquer qu'avec la définition 1.4, le terme $\tilde{I}_{a}\left(x_{a}\right)$ a la structure d'un potentiel à deux corps, et que sous les hypothèses $(H)$ on a, en appliquant les inégalités de Cauchy :

$$
\left|\partial_{x_{a}}^{\alpha} \tilde{I}_{a}\left(x_{a}\right)\right| \leq C_{\alpha}\left\langle x_{a}\right\rangle^{-\rho-|\alpha|}
$$

On se retrouve donc exactement dans le cadre de [I-K], où l'existence de telles fonctions de phase est démontrée.

Nous recollons les deux fonctions de phase ainsi construites en posant :

$$
\phi_{a}\left(x_{a}, \xi_{a}\right)=\chi\left(\cos \left(x_{a}, \xi_{a}\right) \geq \sigma_{a}^{+}\right) \phi_{a}^{+}\left(x_{a}, \xi_{a}\right)+\chi\left(\cos \left(x_{a}, \xi_{a}\right) \leq \sigma_{a}^{-}\right) \phi_{a}^{-}\left(x_{a}, \xi_{a}\right)
$$

et nous définissons le modificateur $J_{a}$ par :

$$
J_{a} f\left(x_{a}, x^{a}\right)=(2 \pi)^{-n} \iint e^{i\left(\phi_{a}\left(x_{a}, \xi_{a}\right)-y_{a}, \xi_{a}\right)} f\left(y_{a}, x^{a}\right) d y_{a} d \xi_{a}
$$

Définition 1.6 - Pour tout canal de réaction $\alpha=\left(a, \psi_{\alpha}, \epsilon_{\alpha}\right)$ et tout intervalle d'énergie $\Delta$ nous définissons les opérateurs d'onde $W_{\alpha}^{ \pm}$par:

$$
W_{\alpha}^{ \pm}=s-\lim _{t \rightarrow \pm \infty} e^{i t H} J_{a} e^{-i t H_{a}} \chi_{a_{0}}\left(\frac{D_{x_{a}}}{\left|D_{x_{a}}\right|}\right) p_{\alpha}
$$

Remarque :

Pour être rigoureux précisons que, en notant $\mathbf{I}_{K_{a}}$ la fonction caractéristique de $K_{a}$, seuls les opérateurs :

$$
W_{\alpha, K_{a}}^{ \pm}=s-\lim _{t \rightarrow \pm \infty} e^{i t H} J_{a} e^{-i t H_{a}} \mathbf{I}_{K_{a}}\left(\frac{D_{x_{a}}}{\left.\mid D_{x_{a}}\right)}\right) p_{\alpha}
$$

ont réellement une signification physique. Ceux-ci se déduisent trivialement de notre définition par la relation :

$$
W_{\alpha, K_{a}}^{ \pm}=W_{\alpha}^{ \pm} \mathbf{I}_{K_{a}}\left(\frac{D_{x_{a}}}{\left|D_{x_{a}}\right|}\right)
$$

la fonction $\chi_{a_{0}}$ que nous avons introduite n'étant qu'une régularisation de $\mathbf{I}_{K_{a}}$. 
Lemme 1.7 - Les opérateurs d'onde ainsi définis existent.

Démonstration :

Comme $\chi_{a_{1}}=1$ sur le support de $\chi_{a_{0}}$, par des arguments de phase non stationnaire on montre aisément que l'on a aussi :

$$
W_{\alpha}^{ \pm}=s-\lim _{t \rightarrow \pm \infty} e^{i t H} J_{a} \chi_{a_{1}}\left(\frac{x_{a}}{\left|x_{a}\right|}\right) e^{-i t H_{a}} \chi_{a_{0}}\left(\frac{D_{x_{a}}}{\left|D_{x_{a}}\right|}\right) p_{\alpha}
$$

Comme

on a encore :

$$
\left[J_{a}, \chi_{a_{1}}\left(\frac{x_{a}}{\left|x_{a}\right|}\right)\right]=O\left(\left\langle x_{a}\right\rangle^{-\rho}\right)
$$

$$
W_{\alpha}^{ \pm}=s-\lim _{t \rightarrow \pm \infty} e^{i t H} \chi_{a_{1}}\left(\frac{x_{a}}{\left|x_{a}\right|}\right) J_{a} e^{-i t H_{a}} \chi_{a_{0}}\left(\frac{D_{x_{a}}}{\left|D_{x_{a}}\right|}\right) p_{\alpha}
$$

Suivant la méthode usuelle nous montrons que cette limite existe en montrant que sur un sous-ensemble dense sa dérivée est intégrable. Pour cela nous calculons :

$$
T_{a}=H \chi_{a_{1}}\left(\frac{x_{a}}{\left|x_{a}\right|}\right) J_{a}-\chi_{a_{1}}\left(\frac{x_{a}}{\left|x_{a}\right|}\right) J_{a} H_{a} p_{\alpha}
$$

Il vient sans difficulté :

$$
\begin{aligned}
T_{a}= & \chi_{a_{1}}\left(\frac{x_{a}}{\left|x_{a}\right|}\right)\left[\left(D_{x_{a}}^{2}+\tilde{I}_{a}\left(x_{a}\right)\right) J_{a}-J_{a} D_{x_{a}}^{2}\right] p_{\alpha} \\
& +\left[D_{x_{a}}^{2}, \chi_{a_{1}}\left(\frac{x_{a}}{\left|x_{a}\right|}\right)\right] J_{a} p_{\alpha} \\
& +\chi_{a_{1}}\left(\frac{x_{a}}{\left|x_{a}\right|}\right)\left(I_{a}(x)-\tilde{I}_{a}\left(x_{a}\right)\right)
\end{aligned}
$$

Le premier terme est à la multiplication par $\chi_{a_{1}}\left(\frac{x_{a}}{\left|x_{a}\right|}\right)$ près le même terme que celui que l'on obtient dans le problème à deux corps. Il est la somme d'un terme de taille $O\left(\left\langle x_{a}\right\rangle^{-1-\rho}\right)$ et d'un terme supporté dans une région $\cos \left(x_{a}, \xi_{a}\right) \in\left[\sigma_{a}^{-}, \sigma_{a}^{+}\right]$. Le deuxième est supporté dans une région $\left|\cos \left(x_{a}, \xi_{a}\right)\right| \leq \sigma_{K_{a}}<1$. Enfin pour le dernier nous remarquons que du fait de la décroissance rapide des états liés :

$$
\left(\tilde{I}_{a}\left(x_{a}\right)-I_{a}(x) \chi_{a_{1}}\left(\frac{x_{a}}{\left|x_{a}\right|}\right) p_{\alpha}=O\left(\left\langle x_{a}\right\rangle^{-1-\nu}\right) \quad, \quad \forall \nu<\rho\right.
$$

de telle sorte que :

$$
T_{a} p_{\alpha}=\left(P_{a}\left(x_{a}, D_{x_{a}}\right)+O\left(\left\langle x_{a}\right\rangle^{-1-\nu}\right)\right) p_{\alpha}
$$

où $P_{a}$ est un opéarteur pseudo-différentiel de symbole supporté dans une région où $\left.\cos \left(x_{a}, \xi_{a}\right) \in\left[\sigma^{-}, \sigma^{+}\right] \subset\right]-1,1[$. 
Or nous savons que pour tout $\epsilon>0$, pour $u$ dans un ensemble dense :

$$
e^{-i t H_{a}} p_{\alpha} u=\chi\left(\left|D_{x_{a}}-\frac{x_{a}}{t}\right| \leq \epsilon\right) e^{-i t H_{a}} p_{\alpha} u+O\left(\langle t\rangle^{-\infty}\right)
$$

L'opérateur $p_{\alpha}$ contenant une troncature supportée dans la région $\left|D_{x_{a}}\right| \geq d-\epsilon_{\alpha}>0$ les termes venant du $O\left(\left\langle x_{a}\right\rangle^{-1-\nu}\right)$ donneront des termes de taille $t^{-1-\nu}$, donc intégrables. Quant au terme $P_{a}\left(x_{a}, D_{x_{a}}\right)$ sa contribution est un $O\left(\langle t\rangle^{-\infty}\right)$, si l'on a choisi $\epsilon$ assez petit, qui est donc aussi intégrable.

Définition 1.8 L'opérateur de diffusion $S_{\alpha, \beta}$ d'un canal $\alpha$ vers un canal $\beta$ est défini par :

$$
p_{\beta}^{*} S_{\alpha, \beta} p_{\alpha}=S_{\alpha, \beta}(\Delta)=\left(W_{\beta}^{+}\right)^{*}\left(W_{\alpha}^{-}\right)
$$

Remarque :

$\mathrm{Du}$ fait de la conservation de l'énergie on peut encore écrire cet opérateur sous forme diagonale à l'aide de $\mathcal{F}_{\alpha}$ et $\mathcal{F}_{\beta}$, les transformées de Fourier associées aux canaux de réaction $\alpha$ et $\beta$, comme suit :

$$
S_{\alpha, \beta}=\int_{\sup \left(\epsilon_{\alpha}, \epsilon_{\beta}\right)}^{+\infty} \mathcal{F}_{\beta}(\lambda)^{*} \mathcal{S}_{\alpha \beta}(\lambda) \mathcal{F}_{\alpha}(\lambda) d \lambda
$$

ce qui définit la matrice de diffusion $\mathcal{S}_{\alpha \beta}(\lambda)$. Nous noterons $S_{\alpha \beta}\left(\lambda, \omega_{a}, \theta_{b}\right)$ son noyau, $\omega_{a}$ et $\theta_{b}$ variant dans les sphères unités $S_{a}$ et $S_{b}$.

\subsection{Résultats :}

Notre premier résultat annonce l'existence d'un prolongement méromorphe de la matrice de diffusion. Nous affirmons que :

Théorème 1.9 - Sous les hypothèses $(H)$ et $(E)$, pour tout compact $K_{b} \subset \tilde{S}_{b}$, il existe un voisinage $\mathcal{V}$ complexe de $[d, M]$ tel que :

(1a) Si $\alpha=\beta$ et si $0<\rho<1, \mathcal{S}_{\alpha \beta}(\lambda)$ se prolonge méromorphiquement sur $\mathcal{V}$ en un opérateur de $G^{s}\left(S_{a}\right)$ dans $G^{s}\left(S_{b}\right)$ et de $G^{s}\left(S_{a}\right)^{\prime}$ dans $G^{s}\left(S_{b}\right)^{\prime}$ pour tout $s \in] 1,(1-\rho)^{-1}[$.

(1b) Si $\alpha=\beta$ et si $\rho \geq 1, \mathcal{S}_{\alpha \beta}(\lambda)$ se prolonge méromorphiquement sur $\mathcal{V}$, en un opérateur de $C^{\infty}\left(S_{a}\right)$ dans $C^{\infty}\left(S_{b}\right)$ et de $D^{\prime}\left(S_{a}\right)$ dans $D^{\prime}\left(S_{b}\right)$.

(2) Si $\alpha \neq \beta$ le noyau de la matrice de diffusion $S_{\alpha \beta}\left(\lambda, \omega_{a}, \theta_{b}\right)$ se prolonge méromorphiquement sur $\mathcal{V}$ en une fonction $G^{s}\left(S_{a} \times \stackrel{\circ}{K_{b}}\right)$, pour tout $s>1$. 
(3) Les pôles de ces prolongements, dans le cas $\alpha=\beta$ comme dans le cas $\alpha \neq \beta$, sont des résonances du hamiltonien $H$ (définies comme étant les pôles du prolongement méromorphe de la résolvante).

Nous précisons maintenant les propriétés du noyau de cette extension méromorphe de la matrice de diffusion :

Théorème 1.10 Supposons les hypothèses $(H)$ et $(E)$ satisfaites, et considérons $\mathcal{V}$ le voisinage complexe de $[d, M]$ introduit dans le théorème précédent. Alors :

(1) Pour tout $\lambda \in \mathcal{V}$ qui n'est pas une résonance du Hamiltonien, le noyau $S_{\alpha \beta}\left(\lambda, \omega_{a}, \theta_{b}\right)$ de la matrice de diffusion est analytique en $\left(\omega_{a}, \theta_{b}\right)$ sur :

- $S_{a} \times S_{a} \cap\left\{\omega_{a} \neq \theta_{b}\right\}$ si $\alpha=\beta$.

- $S_{a} \times \stackrel{\circ}{K}_{b}$ si $\alpha \neq \beta$.

(2) Les résidus de $\mathcal{S}_{\alpha \beta}(\lambda)$ en un pôle $\lambda_{0}$ ont des noyaux analytiques sur $S_{a} \times \stackrel{\circ}{K}_{b}$. 


\section{Une nouvelle construction des opérateurs d'onde.}

La construction des opérateurs d'onde proposée en introduction, qui est le prolongement direct de la construction d'Isozaki-Kitada, est, d'un point de vue technique, loin d'être optimale pour plusieurs raisons.

La première vient $\mathrm{du}$ fait que les estimations que l'on obtient sur $\left(H \chi_{a_{1}}\left(\frac{x_{a}}{\left|x_{a}\right|}\right) J_{a}-\chi_{a_{1}}\left(\frac{x_{a}}{\left|x_{a}\right|}\right) J_{a} H_{a}\right) p_{\alpha}$, qui permettent d'évaluer en quelque sorte la rapidité de convergence des opérateurs d'onde, sont très faibles.

On obtient en effet :

$$
\left(H \chi_{a_{1}}\left(\frac{x_{a}}{\left|x_{a}\right|}\right) J_{a}-\chi_{a_{1}}\left(\frac{x_{a}}{\left|x_{a}\right|}\right) J_{a} H_{a}\right) p_{\alpha}=P_{a}\left(x_{a}, D_{x_{a}}\right)+O\left(\langle x\rangle^{-1-\rho}\right)
$$

où $P_{a}\left(x_{a}, \xi_{a}\right) \quad(i=1,2)$ est un opérateur pseudo-différentiel supporté dans une région où $\cos \left(x_{a}, \xi_{a}\right) \in\left[\sigma_{a}^{-}, \sigma_{a}^{+}\right]$.

Pour démontrer l'existence d'un prolongement méromorphe de la matrice de diffusion, nous aurons besoin en fait d'estimations du type :

$$
\left(H \chi_{a_{1}}\left(\frac{x_{a}}{\left|x_{a}\right|}\right) J_{a}-\chi_{a_{1}}\left(\frac{x_{a}}{\left|x_{a}\right|}\right) J_{a} H_{a}\right) p_{\alpha}=P_{a}\left(x_{a}, D_{x_{a}}\right)+O\left(e^{-\epsilon\langle x\rangle}\right)
$$

où l'opérateur $P_{a}\left(x_{a}, D_{x_{a}}\right)$ sera supporté dans une région sortante pour l'opérateur d'onde entrant et dans une région entrante (ou moins sortante) pour l'opérateur d'onde sortant.

Le deuxième défaut principal de cette construction est que l'on n'a guère de renseignements sur les propriétés d'analyticité des modificateurs introduits, ce qui nous interdit a priori d'utiliser la théorie des distorsions analytiques, essentielle pour arriver aux résultats. 
Ces faits vont nous amener à introduire quelques modifications dans la construction des opérateurs d'onde, tout en imposant bien sûr que leurs valeurs soient inchangées.

La première consiste à définir différemment les opérateurs d'onde entrant et sortant, et ce même dans le cas où les canaux de réaction d'entrée et sortie sont identiques. Le principe de la construction sera cependant toujours le même, seules changeant les propriétés de support des fonctions de troncatures introduites. Pour éviter toute confusion nous ajouterons systématiquement par la suite un indice $j$ aux fonctions de phase, symboles et modificateurs, pour indiquer si l'on traite de l'opérateur d'onde entrant $(j=1)$, ou sortant $(j=2)$.

La deuxième modification consistera à introduire dans la définition des modificateurs un symbole en plus de la fonction de phase.

Enfin, pour pouvoir effectuer par la suite des distorsions analytiques nous prendrons le soin de définir ces symboles et ces fonctions de phase sur un voisinage du réel sur lequel ils seront holomorphes.

De telles modifications sont déjà effectuées pour l'étude du problème à deux corps dans [G-M]. La différence principale qui intervient ici est que nous serons amenés à considérer des symboles à valeur opérateur, afin que leur commutateur avec $H^{a}$ qui intervient dans le calcul $\left(H \tilde{J}_{a}-\tilde{J}_{a} H_{a}\right) p_{\alpha}$ compense les corrections provenant du fait que les amas ne sont pas des particules ponctuelles, c'est à dire le terme $I_{a}(x)-I_{a}\left(x_{a}\right)$. La structure particulière du problème à $N$ corps apparaît donc très clairement à ce niveau, ce qui nous obligera à adopter, nous le verrons, un schéma de construction profondément différent de celui utilisé dans [G-M] pour le problème à deux corps.

\subsection{Construction des opérateurs d'onde pour un canal de réaction à deux amas.}

Nous supposerons dans cette section, et la suivante, que a est une décomposition en deux amas. Nous généraliserons ensuite cette construction aux autres cas dans la section 2.3. Comme annoncé nous choisirons nos modificateurs, que nous noterons $J_{a, j}$, comme étant des opérateurs Fourier intégraux, c'est à dire par :

$$
\begin{aligned}
J_{a, j} f\left(x_{a}, x^{a}\right)= & (2 \pi)^{-n} w_{a}(x) \chi_{a_{1}}\left(\frac{x_{a}}{\left|x_{a}\right|}\right) \\
& \iint e^{i\left(\phi_{a, j}\left(x_{a}, \xi_{a}\right)-y_{a} \cdot \xi_{a}\right)} m_{a, j}\left(x_{a}, \xi_{a}\right) f\left(y_{a}, x^{a}\right) d y_{a} d \xi_{a}
\end{aligned}
$$


où les fonctions de phase $\phi_{a, j}$ et les symboles $m_{a, j}$ restent à définir. Pour arriver à nos fins brièvement exposées ceux ci devront répondre aux trois motivations suivantes :

- $(m 1)$ Pour $M>d>0$ fixés les fonctions $\phi_{a, j}\left(x_{a}, \xi_{a}\right)$ et $m_{a, j}\left(x_{a}, \xi_{a}\right)$ doivent s'étendre à des régions de $\mathbb{C}^{2 n}$ de la forme :

$\left\{\left(x_{a}, \xi_{a}\right)|| x_{a}|>R, d<| \xi_{a} \mid<M\right.$,

$$
\left.\left|\operatorname{Re}\left(x_{a}\right)\right| \leq \epsilon\left|\operatorname{Im}\left(x_{a}\right)\right| \quad\left|\operatorname{Re}\left(\xi_{a}\right)\right| \leq \epsilon\left|\operatorname{Im}\left(\xi_{a}\right)\right|\right\}
$$

et y être holomorphes dans les variables $r_{a}=\left|x_{a}\right|$ et $v_{a}=\left|\xi_{a}\right|$.

- (m2) L'estimation (2.1) doit laisser place à une estimation plus forte du type :

$$
T_{a, j}=P_{j}\left(x_{a}, D_{x_{a}}\right)+O\left(e^{-\epsilon_{0}\left\langle x_{a}\right\rangle}\right)
$$

- (m3) Les opérateurs d'onde doivent être inchangés.

Posons avant tout quelques notations supplémentaires :

Définition 2.1 - Pour $R, d, \epsilon, M$ des réels positifs et $\left.\sigma_{j}^{ \pm} \in\right]-1,1[$ on note :

$$
\begin{gathered}
\mathcal{D}_{a, \epsilon}=\left\{\left(x_{a}, \xi_{a}\right) \in \mathbb{c}^{2 n}|| \operatorname{Im}\left(x_{a}\right)|\leq \epsilon| \operatorname{Re}\left(x_{a}\right)|,| \operatorname{Im}\left(\xi_{a}\right)|\leq \epsilon| \operatorname{Re}\left(\xi_{a}\right) \mid\right\} \\
\Omega_{a}(d, M, \epsilon)=\mathcal{D}_{a, \epsilon} \cap\left\{d<\left|\xi_{a}\right|<M\right\} \\
\Lambda_{a}(R, d, M, \epsilon)=\Omega_{a}(d, M, \epsilon) \cap\left\{\left|x_{a}\right|>R\right\} \\
\Gamma_{a}^{ \pm}\left(R, d, M, \epsilon, \pm \sigma_{j}^{ \pm}\right)=\Lambda_{a}(R, d, M, \epsilon) \cap\left\{ \pm \cos \left(\operatorname{Re}\left(x_{a}\right), \operatorname{Re}\left(\xi_{a}\right)\right) \geq \pm \sigma_{j}^{ \pm}\right\}
\end{gathered}
$$

Nous pouvons alors énoncer le théorème suivant qui répond entre autre à nos trois motivations :

Théorème 2.2 - Pour $0<d<M,-1<\sigma_{j}^{-}<\sigma_{j}^{+}<1$ et $\delta<<1$ fixés il existe des constantes strictement positives $R^{\prime}, \epsilon, \epsilon_{0}, \mu_{0}$, des fonctions de phase $\phi_{a, j}\left(x_{a}, \xi_{a}\right)$ et des symboles $m_{a, j}\left(x_{a}, \xi_{a}\right) \grave{a}$ valeurs dans $B\left(L^{2}\left(X^{a}\right)\right)$, définis sur une région $\mathcal{D}_{a, \epsilon}$ tels que :

(1). $e^{\epsilon_{0}\left\langle x^{a}\right\rangle}\left(D_{x^{a}}\right)^{\nu} m_{a, j}\left(x_{a}, \xi_{a}\right) \in B_{\mu_{0}}\left(L^{2}\left(X^{a}\right)\right) \quad \forall\left(x_{a}, \xi_{a}\right) \in \mathcal{D}_{a, \epsilon}, \nu=0,1$ et

$$
\sup _{\left(x_{a}, \xi_{a}\right) \in \mathcal{D}_{a, \varepsilon}}\left\|e^{\epsilon_{0}\left\langle x^{a}\right\rangle}\left(D_{x^{a}}\right)^{\nu} m_{a, j}\left(x_{a}, \xi_{a}\right)\right\|_{B_{\mu_{0}}\left(L^{2}\left(X^{a}\right)\right)}<+\infty
$$

(2). $e^{\epsilon_{0}\left\langle x^{a}\right\rangle}\left(D_{x^{a}}\right)^{\nu} m_{a, j}\left(x_{a}, \xi_{a}\right)$, pour $\nu=0,1, \phi_{a, j}\left(x_{a}, \xi_{a}\right)$ et leurs dérivées en $x_{a}, \xi_{a}$ sont des fonctions holomorphes en $\left|\xi_{a}\right|$ sur $\Omega_{a}(d, M, \epsilon)$, et holomorphes 
en $\left|x_{a}\right|$ et $\left|\xi_{a}\right|$ sur $\Lambda_{a}\left(R^{\prime}, d, M, \epsilon\right)$.

(3). Les opérateurs d'onde $W_{\alpha}^{ \pm}$définis par :

$$
W_{\alpha}^{ \pm}=s-\lim _{t \rightarrow \pm \infty} e^{i t H} J_{a, j} e^{-i t H_{a}} \chi_{a_{0}}\left(\frac{D_{x_{a}}}{\left|D_{x_{a}}\right|}\right) p_{\alpha}
$$

et

$$
\begin{aligned}
J_{a, j} f\left(x_{a}, x^{a}\right)= & w_{a}(x) \chi_{a_{1}}\left(\frac{x_{a}}{\left|x_{a}\right|}\right)(2 \pi)^{-n_{a}} \\
& \times \iint e^{i\left(\phi_{a, j}\left(x_{a}, \xi_{a}\right)-y_{a} \cdot \xi_{a}\right)} m_{a, j}\left(x_{a}, \xi_{a}\right) f\left(y_{a}, x^{a}\right) d y_{a} d \xi_{a}
\end{aligned}
$$

$\left(w_{a}\right.$ étant une fonction de troncature du type $w_{a}(x)=\chi\left(\cos \left(x_{a}, x\right) \geq \frac{1}{\sqrt{2}}\right)$ ) sont du même type que ceux définis en introduction.

(4). Les symboles $t_{a, j}\left(x_{a}, \xi_{a}\right)$ définis sur $\mathcal{D}_{a, \epsilon}$ par:

$$
\begin{aligned}
t_{a, j}\left(x_{a}, \xi_{a}\right)= & \Delta_{x_{a}} m_{a, j}\left(x_{a}, \xi_{a}\right)+i \nabla_{x_{a}} \phi_{a, j}\left(x_{a}, \xi_{a}\right) . \nabla_{x_{a}} m_{a, j}\left(x_{a}, \xi_{a}\right) \\
& +\left(i \Delta_{x_{a}} \phi_{a, j}\left(x_{a}, \xi_{a}\right)+I_{a}\left(x_{a}\right)-I_{a}(x)-H^{a}+\epsilon_{\alpha}\right) m_{a, j}\left(x_{a}, \xi_{a}\right) \\
& +\left(\xi_{a}^{2}-\left(\nabla_{x_{a}} \phi_{a, j}\left(x_{a}, \xi_{a}\right)\right)^{2}-I_{a}\left(x_{a}\right)\right) m_{a, j}\left(x_{a}, \xi_{a}\right)
\end{aligned}
$$

sont tels que :

$e^{\epsilon_{0}\left\langle x^{a}\right\rangle} t_{a, j}\left(x_{a}, \xi_{a}\right) \in B_{\mu}\left(L^{2}\left(X^{a}\right)\right)$ pour tout $\left(x_{a}, \xi_{a}\right) \in \mathcal{D}_{a, \epsilon}$

La fonction $e^{\epsilon_{0}\left\langle x^{a}\right\rangle} t_{a, j}\left(x_{a}, \xi_{a}\right)$ et ses dérivées en $x_{a}$, $\xi_{a}$ sont des fonctions holomorphes en $\left|\xi_{a}\right|$ sur $\Omega_{a}(d, M, \epsilon)$, et holomorphes en $\left|x_{a}\right|$ et $\left|\xi_{a}\right|$ sur $\Lambda_{a}\left(R^{\prime}, d, M, \epsilon\right)$.

$$
\sup _{\left(x_{a}, \xi_{a}\right) \in \mathcal{D}_{a, \epsilon}}\left\|e^{\epsilon_{0}\left\langle x^{a}\right\rangle} t_{a, j}\left(x_{a}, \xi_{a}\right)\right\|_{B_{\mu}\left(L^{2}\left(X^{a}\right)\right)}<+\infty
$$

et :

$$
\left\|e^{\epsilon_{0}\left\langle x^{a}\right\rangle} t_{a, j}\left(x_{a}, \xi_{a}\right)\right\|_{B_{\mu}\left(L^{2}\left(X^{a}\right)\right)}=O\left(e^{-\epsilon_{0}\left\langle x_{a}\right\rangle}\right)
$$

dans $\Gamma_{a}^{+}\left(R^{\prime}, d, M, \epsilon, \sigma_{j}^{+}\right) \cup \Gamma_{a}^{-}\left(R^{\prime}, d, M, \epsilon,-\sigma_{j}^{-}\right)$

Remarque :

Puisque nous supposons ici que \# $a=2$ la fonction $\chi_{a_{1}}$ qui intervient dans (2.5) est identiquement égale à 1 . Nous l'avons cependant écrite afin d'avoir la même expression lorsque \#a>2.

Pour démontrer ce théorème nous énonçons deux propositions d'existence. La première, traitant des fonctions de phases, est un résultat de [G-M]. 
Proposition 2.3 - Sous les hypothèses $(H)$, pour tout $M_{0}>d_{0}>0$, $\sigma \in]-1,1\left[\right.$ et $\epsilon>0$ assez petit il existe $R_{0}>0$ et deux fonctions $\phi_{a}^{ \pm}\left(x_{a}, \xi_{a}\right)$ holomorphes dans $\Gamma_{a}^{ \pm}=\Gamma_{a}^{ \pm}\left(R_{0}, d_{0}, M_{0}, \epsilon, \sigma\right)$ réelles sur $\Gamma_{a}^{ \pm} \cap \mathbb{R}^{2 n}$ telles que :

$$
\begin{gathered}
\left(\nabla_{x_{a}} \phi_{a}^{ \pm}\left(x_{a}, \xi_{a}\right)\right)^{2}+I_{a}\left(x_{a}\right)=\xi_{a}^{2} \\
\phi_{a}^{ \pm}\left(x_{a}, \xi_{a}\right)-x_{a} \cdot \xi_{a}=O\left(\left\langle x_{a}\right\rangle^{1-\rho}\right)
\end{gathered}
$$

uniformément dans $\Gamma_{a}^{ \pm}$.

Proposition 2.4 - Avec les hypothèses de la proposition précédente et les fonctions de phases $\phi_{a}^{ \pm}\left(x_{a}, \xi_{a}\right)$ qui y sont construites, pour tout $d>d_{0}$, $M<M_{0}, \pm \sigma_{j}^{ \pm}> \pm \sigma$, fixés il existe des constantes $R>R_{0}, \epsilon_{0}>0, \mu>0$ et des fonctions $m_{j}^{ \pm}\left(x_{a}, \xi_{a}\right)$ à valeurs dans $B_{\mu}\left(L^{2}\left(X^{a}\right)\right)$ définies et holomorphes sur $\Gamma_{a}^{ \pm}=\Gamma_{a}^{ \pm}\left(R, d, M, \epsilon, \pm \sigma_{j}^{ \pm}\right)$telles que :

(i) $e^{\epsilon_{0}\left\langle x^{a}\right\rangle}\left(D_{x^{a}}\right)^{\nu} m_{j}^{ \pm}\left(x_{a}, \xi_{a}\right)$, avec $\nu=0,1$, est une fonction holomorphe en $\left(x_{a}, \xi_{a}\right)$ sur $\Gamma_{a}^{ \pm}$et $\left\|m_{j}^{ \pm}\left(x_{a}, \xi_{a}\right)-\pi_{\alpha}\right\|_{B_{\mu}\left(L^{2}\left(X^{a}\right)\right)}=O\left(\left\langle x_{a}\right\rangle^{-\rho}\right)$.

(ii)Si on définit $l_{j}^{ \pm}\left(x_{a}, \xi_{a}\right)$ par :

$$
\begin{aligned}
l_{j}^{ \pm}\left(x_{a}, \xi_{a}\right)= & \left(\Delta_{x_{a}} m_{j}^{ \pm}\left(x_{a}, \xi_{a}\right)+i \nabla_{x_{a}} \phi_{a}^{ \pm}\left(x_{a}, \xi_{a}\right) \cdot \nabla_{x_{a}} m_{j}^{ \pm}\left(x_{a}, \xi_{a}\right)\right. \\
& \left.+\left(i \Delta_{x_{a}} \phi_{a}^{ \pm}\left(x_{a}, \xi_{a}\right)+I_{a}\left(x_{a}\right)-I_{a}(x)-H^{a}+\epsilon_{\alpha}\right) m_{j}^{ \pm}\left(x_{a}, \xi_{a}\right)\right) p_{\alpha}
\end{aligned}
$$

Alors $e^{\epsilon_{0}\left\langle x^{a}\right\rangle}\left(D_{x^{a}}\right)^{\nu} l_{j}^{ \pm}\left(x_{a}, \xi_{a}\right)$ sont des fonctions holomorphes en $\left(x_{a}, \xi_{a}\right)$ sur $\Gamma_{a}^{ \pm}$et vérifient :

$\left\|l_{j}^{ \pm}\left(x_{a}, \xi_{a}\right)\right\|_{B_{\mu}\left(L^{2}\left(X^{a}\right)\right)}=O\left(e^{-\epsilon_{0}\left\langle x_{a}\right\rangle}\right)$ uniformément dans $\Gamma_{a}^{ \pm}$.

Démonstration de la proposition 2.3 :

Il suffit de remarquer que $I_{a}\left(x_{a}\right)$ est un potentiel à deux corps vérifiant les hypothèses $(H)$, et que l'on se trouve par conséquent exactement dans le cadre de la proposition 2.1 de [G-M].

La proposition 2.4 est démontrée dans la section suivante.

Démonstration du théorème 2.2:

Prenons les les fonctions $\Phi^{ \pm}$obtenues par la proposition 2.3 pour $\left.d_{0}=d-\delta,(\delta<<1), \sigma \in\right]-1, \inf \left(-\left|\sigma_{j}^{ \pm}\right|\right)-\delta[$. Nous posons :

$$
\begin{aligned}
\phi_{a, j}\left(x_{a}, \xi_{a}\right)= & \left(\chi\left(\cos \left(x_{a}, \xi_{a}\right) \geq \sigma_{j}^{+}-\delta\right) \phi_{a}^{+}\left(x_{a}, \xi_{a}\right)\right. \\
& \left.+\chi\left(\cos \left(x_{a}, \xi_{a}\right) \leq \sigma_{j}^{-}+\delta\right) \phi_{a}^{-}\left(x_{a}, \xi_{a}\right)\right) \\
& \times \chi\left(\left|\operatorname{Re}\left(x_{a}\right)\right| \geq R_{0}\right) \chi\left(d-\delta<\left|\operatorname{Re}\left(\xi_{a}\right)\right|<M+\delta\right)
\end{aligned}
$$

qui sont des fonctions de phase vérifiant la condition 2 du théorème. On remarque aussi que les fonctions $\phi_{a}^{ \pm}$étant solutions de l'équation eikonale 
le dernier membre de $(2.6)$ est nul dans $\Gamma_{a}^{ \pm}\left(R^{\prime}, d, M, \epsilon, \pm \sigma_{j}^{ \pm}\right)$, pour tout $R^{\prime}>R_{0}$.

Pour ce qui est des symboles $m_{a, j}\left(x_{a}, \xi_{a}\right)$, nous recollons les symboles $m_{j}^{+}\left(x_{a}, \xi_{a}\right)$ et $m_{j}^{-}\left(x_{a}, \xi_{a}\right)$ donnés par la proposition 2.4 en posant :

$$
\begin{aligned}
m_{a, j}\left(x_{a}, \xi_{a}\right)= & {\left[\chi\left(\cos \left(x_{a}, \xi_{a}\right)>\sigma_{j}^{+}\right) m_{j}^{+}\left(x_{a}, \xi_{a}\right)\right.} \\
& \left.+\chi\left(\cos \left(x_{a}, \xi_{a}\right)<\sigma_{j}^{-}\right) m_{j}^{-}\left(x_{a}, \xi_{a}\right)\right] \\
& \times \chi\left(d \leq\left|\operatorname{Re}\left(\xi_{a}\right)\right| \leq M\right) \chi\left(\left|\operatorname{Re}\left(x_{a}\right)\right|>R^{\prime}\right)
\end{aligned}
$$

Le symbole ainsi défini, et ses dérivées par rapport à $x_{a}$ et $\xi_{a}$ sont bien holomorphes en $\left|\xi_{a}\right|$ dans $\Omega_{a}(d, M, \epsilon)$, et en $\left|x_{a}\right|$ et $\left|\xi_{a}\right|$ dans la région $\Lambda_{a}(R, d, M, \epsilon)$.

De plus, d'après le $(i)$ de la proposition il est aisé de vérifier que les opérateurs d'onde sont inchangés. Enfin le point (4) du théorème suit directement de $(i i)$ et du fait que, comme nous l'avons remarqué, le dernier terme de (2.6) est nul sur le support de $m_{a, j}$.

Remarque : Les symboles ainsi définis ne sont pas analytiques en $\frac{x_{a}}{\left|x_{a}\right|}$ et $\frac{\xi_{a}}{\left|\xi_{a}\right|}$ à cause des fonctions de troncature $\chi\left(\cos \left(x_{a}, \xi_{a}\right)\right)$ que nous avons introduites. Nous sommes cependant libres d'imposer à ces fonctions la régularité que l'on souhaite, régularité que l'on sera amené quelquefois à préciser par la suite.

\subsection{Démonstration de la proposition 2.4}

Commençons par établir quelques estimations.

Lemme 2.5 Notons $q=n(N-2)=\operatorname{dim} X^{a}$, et $\Gamma_{\alpha}=\left(H^{a}-\epsilon_{\alpha}\right)^{-1}\left(1-\pi_{\alpha}\right)$.

Sous les hypothèses de la proposition 2.4, il existe $\mu>0$ tel que pour tout $l \in \mathbb{N}$, tout multi-indice $\tau \in \mathbb{N}^{l}$ et tout $l$-uplet de q-indices , $\kappa \in\left(N^{q}\right)^{l}$, les opérateurs, que nous noterons $\left(D_{x^{a}}\right)^{\nu}\left(\Gamma_{\alpha}\right)^{\tau}\left(x^{a}\right)^{\kappa} \pi_{\alpha}$, définis pour $\nu=0,1$ par :

$$
\left(D_{x^{a}}\right)^{\nu}\left(\Gamma_{\alpha}\right)^{\tau}\left(x^{a}\right)^{\kappa} \pi_{\alpha}:=\left(D_{x^{a}}\right)^{\nu}\left(\prod_{i=1}^{l}\left(\Gamma_{\alpha}\right)^{\tau_{i}}\left(x^{a}\right)^{\kappa_{i}}\right) \pi_{\alpha}
$$

sont des opérateurs bornés $\mu$-analytiquement dilatables.

De plus, il existe des constantes $\epsilon_{0}$ et $C_{0}$, indépendantes des multi-indices $\kappa$ et $\tau$ telles que pour $\nu=0,1$ :

$$
\left\|e^{\epsilon_{0}\left\langle x^{a}\right\rangle}\left(D_{x^{a}}\right)^{\nu} \Gamma_{\alpha}^{\tau}\left(x^{a}\right)^{\kappa} \pi_{\alpha}\right\|_{B_{\mu}\left(L^{2}\left(X^{a}\right)\right)} \leq C_{0}^{\frac{|\kappa|+|\tau|}{2}}|\kappa|^{|\kappa|}
$$


Remarques sur les notations :

La valeur zéro étant permise pour les coefficients $\tau_{i}$ et $\kappa_{i}$ plusieurs multiindices peuvent définir les mêmes opérateurs $\left(\Gamma_{\alpha}\right)^{\tau}\left(x^{a}\right)^{\kappa} \pi_{\alpha}$. Pour des raisons d' écriture on supposera dans la démonstration, sans perte de généralité, que $\tau_{1}=0$ et $\tau_{i}=1$, pour tout $0<i \leq l$.

Démonstration du lemme 2.5:

Commençons par démontrer (2.11) dans le cas $\epsilon_{0}=0$.

$\epsilon_{\alpha}$ étant une valeur propre isolée de $H^{a}$, pour un contour $\Xi$ suffisamment proche de $\epsilon_{\alpha}$ on a :

$$
\Gamma_{\alpha}=\left(H^{a}-\epsilon_{\alpha}\right)^{-1}\left(1-\pi_{\alpha}\right)=\frac{1}{2 \pi i} \int_{\Xi} \frac{d z}{\left(z-\epsilon_{\alpha}\right)\left(z-H^{a}\right)}
$$

et $\left(\Gamma_{\alpha}\right)^{\tau}\left(x^{a}\right)^{\kappa} \pi_{\alpha}=\int_{\Xi \times \cdots \times \Xi} d z_{2} \cdots d z_{l}$

$$
\left(x^{a}\right)^{\kappa_{1}} \frac{1}{\left(z_{2}-\epsilon_{\alpha}\right)\left(z_{2}-H^{a}\right)}\left(x^{a}\right)^{\kappa_{2}} \cdots \frac{1}{\left(z_{l}-\epsilon_{\alpha}\right)\left(z_{l}-H^{a}\right)}\left(x^{a}\right)^{\kappa_{l}} \pi_{\alpha}
$$

Il nous suffit donc d' étudier des termes de la forme $\left(D_{x^{a}}\right)^{\nu} M_{\tau \kappa}$ où :

$$
M_{\tau \kappa}=\left(x^{a}\right)^{\kappa_{1}} \frac{1}{\left(z_{2}-H^{a}\right)}\left(x^{a}\right)^{\kappa_{2}} \cdots \frac{1}{\left(z_{l}-H^{a}\right)}\left(x^{a}\right)^{\kappa_{l}} \pi_{\alpha}
$$

L'idée générale consiste à faire commuter les termes $\left(x^{a}\right)^{\kappa_{i}}$ et les termes $\frac{1}{\left(z_{j}-H^{a}\right)}$, de façon à obtenir une expression où toutes les puissances de $x^{a}$ sont à droite, afin de pouvoir utiliser les estimations connues sur les états liés, et leur décroissance exponentielle en particulier. Le facteur $\left(D_{x^{a}}\right)^{\nu}$ à gauche ne posera aucun problème dès que l'on remarque que $\left\langle D_{x^{a}}^{2}\right\rangle \frac{1}{\left(z_{j}-H^{a}\right)}$ est un opérateur borné. Encore faut-il s'assurer qu'il y a bien au moins un terme de la forme $\frac{1}{\left(z_{j}-H^{a}\right)}$ dans l'expression de $M_{\tau \kappa}($ attention au cas $l=1)$ ce que l'on fait en écrivant artificiellement $\pi_{\alpha}=\frac{i-\epsilon_{\alpha}}{i-H^{a}} \pi_{\alpha}$.

Venons-en donc au calcul des commutateurs. Pour $p \in \mathbb{N}^{q}$ posons $I_{p}=\left(x^{a}\right)^{p}\left(H^{a}-z\right)^{-1}$ et adoptons la convention $I_{p}=0$ si $p \in \mathbb{Z}^{q} \backslash \mathbb{N}^{q}$. Il vient :

$$
\begin{aligned}
I_{p}= & \left(H^{a}-z\right)^{-1}\left(x^{a}\right)^{p}-\sum_{\sigma=1}^{q} \frac{i}{2} p_{\sigma}\left(H^{a}-z\right)^{-1}\left(D_{x^{a}}\right)_{\sigma} I_{p-1_{\sigma}} \\
& +\sum_{\sigma=1}^{q} \frac{p_{\sigma}\left(p_{\sigma}-1\right)}{2}\left(H^{a}-z\right)^{-1} I_{p-2_{\sigma}}
\end{aligned}
$$

En itérant il est clair que l'on obtient pour finir une expression où toutes les puissances de $x^{a}$ sont à droite. Plus précisément :

$$
I_{p}=\sum_{i, j \in \omega}\left(-\frac{i}{2}\right)^{|i|}\left(\frac{1}{2}\right)^{j} \frac{p !}{(p-i-2 j) !}\left(\left(H^{a}-z\right)^{-1}\left(D_{x^{a}}\right)\right)^{i}\left(H^{a}-z\right)^{-j}\left(x^{a}\right)^{p-i-2 j}
$$


où $\omega$ est un sous ensemble de $\left\{i, j \in \mathbb{N}^{q} \mid 0<i+2 j \leq p\right\}$. Ainsi $I_{p}$ s'écrit de la forme :

$$
I_{p}=\sum_{\substack{k \in \mathbb{N}^{q} \\ k \leq p}} B_{k}\left(x^{a}\right)^{(p-k)}
$$

avec $\left\langle D_{x^{a}}\right\rangle B_{k} \in B\left(L^{2}\left(X^{a}\right)\right)$ qui sont des produits d'opérateurs $\mu$-analytiquement dilatables, pour $\mu$ assez petit, et $\left\|\left\langle D_{x^{a}}\right\rangle B_{k}\right\|_{B_{\mu}\left(L^{2}\left(X^{a}\right)\right)} \leq C^{|k|} \frac{p !}{(p-k) !}$. De plus au regard de l'expression (2.12) il est clair que le nombre de termes non nuls d'ordre $|k|$ intervenant dans la somme ci dessus est majoré par $(2 q)^{|k|}$. On peut ainsi écrire :

$$
I_{p}=\sum_{k=0}^{|p|} B_{k}^{\prime}\left(x^{a}\right)^{p-k} ;\left\|\left(D_{x^{a}}\right)^{\nu} B_{k}^{\prime}\right\|_{B_{\mu}\left(L^{2}\left(X^{a}\right)\right)} \leq\left(C_{1}\right)^{k} \frac{|p| !}{(|p|-k) !} ; \nu=0,1
$$

Évaluons maintenant les termes $M_{\tau \kappa}$. Etant entendu qu'on ne considère ici que les cas où $\tau=(0,1, \cdots, 1)$ on notera sans que cela porte à confusion $M_{\tau \kappa}=M\left(\kappa_{1}, \cdots, \kappa_{l}\right)$. D'après $(2.13)$ on a l'égalité suivante :

$$
\left(D_{x^{a}}\right)^{\nu} M\left(\kappa_{1}, \kappa_{2}, \kappa_{3}, \cdots, \kappa_{l}\right)=\sum_{j_{1}=0}^{\kappa_{1}} B_{j_{1}}^{1} M\left(0, \kappa_{1}-j_{1}+\kappa_{2}, \kappa_{3}, \cdots, \kappa_{l}\right)
$$

où les $B_{j_{1}}^{1}$, sont des opérateurs bornés $\mu$-analytiquement dilatables de norme majorée par $\left(C_{1}\right)^{j_{1}} \frac{\left|\kappa_{1}\right| !}{\left(\left|\kappa_{1}\right|-j_{1}\right) !}$. Ainsi par itération :

$$
\begin{aligned}
M\left(\kappa_{1}, \kappa_{2}, \cdots, \kappa_{l}\right)= & \sum_{j_{1}=0}^{\left|\kappa_{1}\right|} \sum_{j_{2}=0}^{\left|\kappa_{2}\right|+\left(\left|\kappa_{1}\right|-j_{1}\right)} \ldots \\
& \sum_{j_{l}=0}^{\left|\kappa_{l}\right|+\left(\left|\kappa_{1}\right|-j_{1}\right)+\cdots+\left(\left|\kappa_{l-1}\right|-j_{l-1}\right)} \prod_{s=1}^{l} B_{j_{s}}\left(x^{a}\right)^{(|\kappa|-|j|)} \pi_{\alpha}
\end{aligned}
$$

les opérateurs $B_{j_{s}}$ vérifiant :

$$
\left\|B_{j_{s}}\right\|_{B_{\mu}\left(L^{2}\left(X^{a}\right)\right)} \leq\left(C_{1}\right)^{j_{s}} \frac{\left(\sum_{i=1}^{s-1}\left(\left|\kappa_{i}\right|-j_{i}\right)+\kappa_{s}\right) !}{\left(\sum_{i=1}^{s}\left(\left|\kappa_{i}\right|-j_{i}\right)\right) !}
$$

où l'on a pris la convention $j_{0}=0$ et $\kappa_{0}=0$.

Nous sommes maintenant en mesure d'achever la démonstration du lemme. D'après des résultats classiques sur les états liés d'énergie négative (Cf [Co-T] Théorème 1 ) on sait qu'il existe $\mu>0$ assez petit et $C>0$ tels que :

$$
|\operatorname{Im}(\theta)|<\mu \Rightarrow e^{\left\langle x^{a}\right\rangle / C} U_{\theta}\left(\psi_{\alpha}\right) \in L^{2}\left(X^{a}\right)
$$

Ainsi pour tout $m \in \mathbb{N}^{q}\left(x^{a}\right)^{m} \psi_{\alpha}$ est un vecteur $\mu$-analytiquement dilatable de norme $L^{2}\left(X^{a}\right)_{\mu}$ majorée par $C^{m}|m|$ ! et l'égalité (2.14) montre directement que les opérateurs $M_{\tau \kappa}$ sont $\mu$-analytiquement dilatables et bornés. Il 
ne reste donc plus qu'à évaluer leur norme. En remarquant que pour tout indice $s$ :

$$
\frac{\left(\sum_{i=1}^{s-1}\left(\left|\kappa_{i}\right|-j_{i}\right)+\kappa_{s}\right) !}{\left(\sum_{i=1}^{s-1}\left(\left|\kappa_{i}\right|-j_{i}\right)\right) !} \leq \frac{\left(\sum_{i=1}^{s-1}\left|\kappa_{i}\right|+\left|\kappa_{s}\right|\right) !}{\left(\sum_{i=1}^{s-1}\left|\kappa_{i}\right|\right) !}=\frac{\left(\sum_{i=1}^{s}\left|\kappa_{i}\right|\right) !}{\left(\sum_{i=1}^{s-1}\left|\kappa_{i}\right|\right) !}
$$

on vérifie aisément la majoration :

$$
\prod_{s=1}^{l-1} \frac{\left(\sum_{i=1}^{s-1}\left(\left|\kappa_{i}\right|-j_{i}\right)+\kappa_{s}\right) !}{\left(\sum_{i=1}^{s}\left(\left|\kappa_{i}\right|-j_{i}\right)\right) !} \leq \frac{|\kappa| !}{(|\kappa|-|j|) !}
$$

Ainsi en utilisant (2.15), et en remarquant que $j_{1}+\cdots+j_{l-1} \leq|\kappa|$, on obtient :

$$
\begin{aligned}
\left\|\left(D_{x^{a}}\right)^{\nu} M_{\tau \kappa}\right\|_{B_{\mu}\left(L^{2}\left(X^{a}\right)\right) \leq} & C^{|\kappa|}|\kappa| ! \sum_{j_{1}=0}^{\left|\kappa_{1}\right|} \sum_{j_{2}=0}^{\left|\kappa_{2}\right|+\left(\left|\kappa_{1}\right|-j_{1}\right)} \ldots \\
& \sum_{j_{l}=0}^{\left|\kappa_{l}\right|+\left(\kappa_{1} \mid-j_{1}\right)+\cdots+\left(\left|\kappa_{l-1}\right|-j_{l-1}\right)} \prod_{s=1}^{l} C_{1}^{j_{s}} C^{-j_{s}}
\end{aligned}
$$

En prenant la constante $C$ strictement supérieure à $C_{1}$ toutes les sommes apparaissant ci dessus sont majorées par $\frac{1}{1-\frac{C_{1}}{C}}$ et il vient pour finir :

$$
\left\|\left(D_{x^{a}}\right)^{\nu} M_{\tau \kappa}\right\|_{B_{\mu}\left(L^{2}\left(X^{a}\right)\right)} \leq C^{|\kappa|}|\kappa|\left(\frac{1}{1-\frac{C_{1}}{C}}\right)^{|\tau|+1}
$$

ce qui donne l'estimation (2.11) souhaitée, pour $\epsilon_{0}=0$.

Montrons alors que l'on peut généraliser cette estimation à un $\epsilon_{0}>0$. D'après l'estimation obtenue ci-dessus on sait qu'il existe $K>0$ tel que pour tout multi-indice $\kappa$ et $\tau$ :

$$
\left\|\left(D_{x^{a}}\right)^{\nu} \Gamma_{\alpha}^{\tau}\left(x^{a}\right)^{\kappa} \pi_{\alpha}\right\|_{B_{\mu}\left(L^{2}\left(X^{a}\right)\right)} \leq K^{|\kappa|+|\tau|}|\kappa| ! \quad ; \quad \nu=0,1
$$

En développant $e^{\epsilon_{0}\left\langle x^{a}\right\rangle}$ en série entière il vient pour $K \epsilon_{0}<1$ :

$$
\begin{aligned}
\left\|\left(D_{x^{a}}\right)^{\nu} e^{\epsilon_{0}\left\langle x^{a}\right\rangle} \Gamma_{\alpha}^{\tau}\left(x^{a}\right)^{\kappa} \pi_{\alpha}\right\|_{B_{\mu}\left(L^{2}\left(X^{a}\right)\right)} & \leq \sum_{i=0}^{\infty} \frac{(|\kappa|+i) !}{i !} K^{|\kappa|+i+|\tau|} \epsilon_{0}^{i} \\
& =K^{|\kappa|+|\tau|}|\kappa| !\left(\frac{1}{1-K \epsilon_{0}}\right)^{|\kappa|+1}
\end{aligned}
$$

Il ne reste plus qu'à constater que $\left[e^{\epsilon_{0}\left\langle x^{a}\right\rangle}, D_{x^{a}}\right]=i \epsilon_{0} \frac{x^{a}}{\left\langle x^{a}\right\rangle} e^{\epsilon_{0}\left\langle x^{a}\right\rangle}$ pour achever la démonstration du lemme.

Démonstration de la proposition 2.4 :

Nous limiterons notre démonstration au cas $\Gamma^{+}\left(R, d, M, \epsilon, \sigma_{j}^{+}\right)$le cas $\Gamma^{-}\left(R, d, M, \epsilon,-\sigma_{j}^{-}\right)$étant tout à fait similaire. Afin d'alléger les notations nous omettrons les indices $a, j$ tout au long de la démonstration. 
Nous chercherons des fonctions vérifiant les conditions de la proposition sous la forme $m=\tilde{m}+\tilde{n}$ avec :

$$
\tilde{m}=\pi_{\alpha} m \text { et } \tilde{n}=\left(1-\pi_{\alpha}\right) m
$$

En posant $\lambda^{\alpha}=\left(\psi_{\alpha}\left(x^{a}\right), x^{a} \psi_{\alpha}\left(x^{a}\right)\right)$ la condition (ii) s'écrit alors :

$$
(I)\left\{\begin{array}{c}
i \nabla_{x_{a}} \phi \cdot \nabla_{x_{a}} \tilde{m}+\left(i \Delta_{x_{a}} \phi-\lambda^{\alpha} \cdot \nabla^{a} I_{a}\left(x_{a}\right)\right) \tilde{m}=-\Delta_{x_{a}} \tilde{m} \\
\quad+\pi_{\alpha}\left(I_{a}(x)-I_{a}\left(x_{a}\right)-x^{a} \cdot \nabla^{a} I_{a}\left(x_{a}\right)\right) \pi_{\alpha} \tilde{m}+\pi_{\alpha}\left(I_{a}(x)-I_{a}\left(x_{a}\right)\right) \tilde{n} \\
\left(H^{a}-\epsilon_{\alpha}\right) \tilde{n}=\left(\nabla_{x_{a}} \phi \cdot \nabla_{x_{a}} \tilde{n}+i \Delta_{x_{a}} \phi \tilde{n}+\Delta_{x_{a}} \tilde{n}\right) \\
-\left(1-\pi_{\alpha}\right)\left(I_{a}(x)-I_{a}\left(x_{a}\right)\right)(\tilde{m}+\tilde{n})
\end{array}\right.
$$

modulo des termes qui sont des $O\left(e^{-\epsilon\langle x\rangle}\right)$.

Nous chercherons des solutions exactes de $(I)$ sous la forme de séries formelles $\tilde{n}=\sum_{k \geq 0} \tilde{n}_{k}$ et $\tilde{m}=\sum_{k \geq 0} \tilde{m}_{k}$, que nous resommerons par la suite. Pour pouvoir obtenir des symboles analytiques après resommation nous devons contrôler ici précisément les normes des $\tilde{n}_{k}$ et $\tilde{m}_{k}$ en fonction de $k$ . Pour cela une construction de la solution par itération est peu adaptée et il nous est apparu préférable d'utiliser pour résoudre $(I)$ un théorème $\mathrm{du}$ point fixe, dans un espace de Banach que nous allons définir ci-dessous.

De façon analogue à la technique développée dans [G-M] on définit sur $\Gamma$ la fonction $d\left(x_{a}, \xi_{a}\right)$ par :

$$
\begin{aligned}
d\left(x_{a}, \xi_{a}\right)= & \left(\operatorname{Re}\left(x_{a}\right) \cdot \operatorname{Re}\left(\xi_{a}\right)\right)-r_{1}\left|\operatorname{Re}\left(\xi_{a}\right)\right| \\
& +s\left|\operatorname{Re}\left(x_{a}\right)\right| \cdot\left|\operatorname{Re}\left(\xi_{a}\right)\right|-r_{0}\left|\operatorname{Im}\left(x_{a}\right)\right| \cdot\left|\operatorname{Re}\left(\xi_{a}\right)\right|
\end{aligned}
$$

où les constantes $r_{1}, s, r_{0}$ sont des réels choisis à l'aide de la proposition suivante :

Proposition 2.6 - Pour $\left.0<d<M, \epsilon_{0}>0, \sigma^{+} \in\right]-1,1[$ et $\delta>0$ fixés, il existe $r_{1}, s, r_{0} \in \mathbb{R}$ et $\epsilon, \nu>0$ tels que, en notant:

$$
\begin{gathered}
\Omega_{\xi_{a}}=\left\{x_{a} \mid d\left(x_{a}, \xi_{a}\right) \geq 0\right\} \\
\Omega=\left\{\left(x_{a}, \xi_{a}\right) \mid\left(x_{a}, \xi_{a}\right) \in \mathcal{D}_{a, \epsilon}, x_{a} \in \Omega_{\xi_{a}}\right\}
\end{gathered}
$$

on ait :

$$
\begin{gathered}
\Gamma^{+}\left(R, d, M, \epsilon, \sigma^{+}\right) \subset \Omega \subset \Gamma^{+}\left(R, d, M, \epsilon_{0}, \sigma^{+}-\delta\right) \\
\frac{\partial \operatorname{Re}(\phi)}{\partial \operatorname{Re}\left(x_{a}\right)} \cdot \frac{\partial d}{\partial \operatorname{Re}\left(x_{a}\right)}-\frac{\partial \operatorname{Im}(\phi)}{\partial \operatorname{Im}\left(x_{a}\right)} \cdot \frac{\partial d}{\partial \operatorname{Im}\left(x_{a}\right)} \geq \nu \quad, \quad \forall\left(x_{a}, \xi_{a}\right) \in \Omega \\
\nu\left\langle x_{a}\right\rangle \leq d\left(x_{a}, \xi_{a}\right) \leq \frac{1}{\nu}\left\langle x_{a}\right\rangle \quad, \quad \forall\left(x_{a}, \xi_{a}\right) \in \Gamma^{+}\left(R, d, M, \epsilon, \sigma^{+}\right) \\
\nu \operatorname{dist}\left(x_{a}, \partial \Omega_{\xi_{a}}\right) \leq d\left(x_{a}, \xi_{a}\right) \leq \frac{1}{\nu} \operatorname{dist}\left(x_{a}, \partial \Omega_{\xi_{a}}\right) \quad, \quad \forall\left(x_{a}, \xi_{a}\right) \in \Omega
\end{gathered}
$$


Le lecteur souhaitant trouver la preuve de cette proposition pourra se reporter directement au lemme 3.2 de [G-M].

On note $E_{k}^{1}$ les espaces vectoriels normés définis par :

$$
\begin{aligned}
E_{k}^{1}= & \{m \in H(\Omega, c) \mid \exists f(m, k) \in \mathbb{R} \text { tel que } \\
& \left.\left|m\left(x_{a}, \xi_{a}\right)\right| \leq\left(C_{0} k\right)^{k}\left\langle x_{a}\right)^{-\rho} d\left(x_{a}, \xi_{a}\right)^{-k} f(m, k)\right\}
\end{aligned}
$$

la norme $\|m\|_{E_{k}^{1}}$ étant donnée par la meilleure constante $f(m, k)$ que l'on peut prendre dans (2.22) et $C_{0}$ étant la constante intervenant dans le lemme 2.5 .

On note $\tilde{E}_{k}^{2}$ les espaces vectoriels $E_{k}^{1}$ munis de la norme $\|\cdot\|_{\tilde{E}_{k}^{2}}=\left(k C_{0}\right)^{k}\|\cdot\|_{E_{k}^{1}}$.

Pour $k>0$ on définit $F_{k}$ comme étant l'espace vectoriel des polynômes à deux variables $X$ et $Y$ qui ne commutent pas, de degrés partiels en $X$ et en $Y$ inférieurs ou égaux à $k$. En notant :

$$
\left(X^{\tau} Y^{\kappa}\right)=\prod_{i=1}^{k} X^{\kappa_{i}} Y^{\tau_{i}}=X^{\tau_{1}} Y^{\kappa_{1}} \cdots X^{\tau_{k}} Y^{\kappa_{k}}
$$

les vecteurs de la base canonique on définit :

$$
\left\|\left(X^{\tau} Y^{\kappa}\right)\right\|_{F_{k}}=C_{0}^{\left(\frac{|\tau|+|\kappa|}{2}-k\right)} k^{(|\kappa|-k)}
$$

et on munit $F_{k}$ de la norme donnée par :

$$
\left\|\sum C_{\tau, \kappa} X^{\tau} Y^{\kappa}\right\|_{F_{k}}=\sum\left|C_{\tau, \kappa}\right|\left\|X^{\tau} Y^{\kappa}\right\|_{F_{k}}
$$

On pose :

$$
E_{k}^{2}=\tilde{E}_{k}^{2} \otimes F_{k}
$$

$E_{k}^{2}$ étant muni de la norme produit $\|\cdot\|_{\tilde{E}_{k}^{2}} \times\|\cdot\|_{F_{k}}$ On définit enfin pour tout $\mu>0$ les espaces vectoriels :

$$
\begin{gathered}
E_{\mu}^{1}=\left\{m=\left(m_{k}\right)_{k \in \mathbb{N}} \mid m_{k} \in E_{k}^{1} \text { et }\|m\|_{E_{\mu}^{1}}=\sum_{k \geq 0} \mu^{k}\left\|m_{k}\right\|_{E_{k}^{1}}<+\infty\right\} \\
E_{\mu}^{2}=\left\{n=\left(n_{k}\right)_{k \in \mathbb{N}} \mid n_{k} \in E_{k}^{2} \text { et }\|n\|_{E_{\mu}^{2}}=\sum_{k \geq 0} \mu^{k-1}\left\|n_{k}\right\|_{E_{k}^{2}}<+\infty\right\}
\end{gathered}
$$

qui sont des espaces de Banach. L'espace sur lequel nous utiliserons un théorème du point fixe sera $E_{\mu}=E_{\mu}^{1} \times E_{\mu}^{2}$

Remarque :

Si elle peut paraître abstraite la définition des espaces $E_{\mu}^{1}$ et $E_{\mu}^{2}$ est en fait 
assez naturelle car, si l'on essaie de résoudre le système $(I)$ par itération, on voit que les $\tilde{m}_{k}$ (resp. $\tilde{n}_{k}$ ) s' écrivent comme des termes $p_{k} \otimes \pi_{\alpha}$ (resp. des sommes de termes $p_{k} \otimes M_{k} \pi_{\alpha}$ ) où les $p_{k}$ sont des symboles de taille $\langle x\rangle^{-k-\rho}$ et les $M_{k}$ des opérateurs de la forme $\left(\left(\Gamma_{\alpha}\right)^{\tau}\left(x^{a}\right)^{\kappa}\right)$, avec $|\tau| \leq k$ et $|\kappa| \leq k$. La définition des espaces $E_{\mu}^{1}$ et $E_{\mu}^{2}$ n'est que le reflet de cette structure, et l'on peut garder à l'esprit pour la suite que les variables $X$ et $Y$ seront à la fin prises égales à $\Gamma_{\alpha}$ et $x^{a}$. Notons enfin ce qui a motivé l'introduction de ces espaces abstraits $F_{k}$ : les opérateurs de multiplication $\operatorname{par}\left(X^{\tau} Y^{\kappa}\right)$ de $F_{k}$ dans $F_{k+i}$ ont des normes faciles à évaluer ce qui n'est pas le cas des opérateurs de multiplication par $\left(\left(\Gamma_{\alpha}\right)^{\tau}\left(x^{a}\right)^{\kappa}\right)$ sur les sous-espaces de $B\left(L^{2}\left(X^{a}\right)\right)$ que l'on aurait été amené à considérer.

Dans la suite nous noterons $\mathcal{P}_{a}$ l'application définie par :

$$
\mathcal{P}_{a}: \begin{cases}F_{k} & \rightarrow \mathfrak{c} \\ P(X, Y) & \rightarrow\left(\psi_{\alpha}, P\left(\Gamma_{\alpha}, x^{a}\right) \psi_{\alpha}\right)_{L^{2}\left(X^{a}\right)}\end{cases}
$$

et nous noterons de même, sans que cela porte à confusion l'application $1 \otimes \mathcal{P}_{a}$ de $E_{k}^{2}$ dans $E_{k}^{1}$.

On se propose maintenant de résoudre dans les espaces de Banach que l'on vient de définir le système suivant, qui est l'analogue du système $(I)$ et de la condition $(i)$ :

$$
\left(I_{1}\right)\left\{\begin{aligned}
\tilde{m}-1 \in & E_{\mu}^{1} \\
\tilde{n} \in & E_{\mu}^{2} \\
R_{o} \tilde{m}= & -\Delta_{x_{a}} \tilde{m}+\sum_{i=2}^{+\infty} \mathcal{P}_{a}\left(Y^{i}\right) \cdot \frac{\left(\nabla^{a}\right)^{i}\left(I_{a}\right)\left(x_{a}\right)}{i !} \tilde{m} \\
& \left.+\sum_{i=1}^{+\infty} \mathcal{P}_{a} \frac{Y^{i} \cdot\left(\nabla^{a}\right)^{i}\left(I_{a}\right)\left(x_{a}\right)}{i !} \tilde{n}\right] \\
\tilde{n}= & X R_{1} \tilde{n} \\
& +\sum_{i=1}^{+\infty} \frac{X Y^{i} \cdot\left(\nabla^{a}\right)^{i}\left(I_{a}\right)\left(x_{a}\right)}{i !}(\tilde{n}+\tilde{m})
\end{aligned}\right.
$$

où l'on a posé

$$
\left\{\begin{array}{l}
R_{1} m=i\left(\nabla_{x_{a}} \phi \nabla_{x_{a}} m+\Delta_{x_{a}} \phi m\right) \\
R_{0} m=R_{1} m-\lambda^{\alpha} . \nabla_{x^{a}} I_{a}\left(x_{a}\right) m
\end{array}\right.
$$

Nous montrerons dans un premier temps que ce système a bien un sens, c'est à dire que les sommes infinies sont convergentes, et nous en construirons ensuite une solution.

Tout d'abord énonçons quelques propriétés : 
Lemme 2.7:

(a) Pour tout polynôme $P(X, Y)$ de $F_{k}$ on a :

$$
\left|\mathcal{P}_{a}\left(Y^{i} P(X, Y)\right)\right| \leq C_{0}^{k+i}(k+i)^{k+i}\|P(X, Y)\|_{F_{k}}
$$

(b) La dérivation par rapport à une composante de $x_{a}$, $\partial_{x_{a}}$, est une opération continue de $E_{k}^{1}$ dans $E_{k+1}^{1}$, pour tout $k \in \mathbb{N}$ et de norme majorée uniformément par rapport ’̀ $k$.

(c)Il existe $C, C_{1} \in \mathbb{R}$ telles que $\forall\left(x_{a}, \xi_{a}\right) \in \Omega$ :

$$
\left|\left(\nabla^{a}\right)^{i} I_{a}\left(x_{a}\right)\right| \leq C^{i}(i !)\left\langle x_{a}\right\rangle^{-\rho} d\left(x_{a}, \xi_{a}\right)^{-i}
$$

et

$$
\left|\partial_{x_{a}}\left(\phi\left(x_{a}, \xi_{a}\right)-x_{a} \cdot \xi_{a}\right)\right| \leq C_{1}^{|\alpha|} \alpha ! d\left(x_{a}, \xi_{a}\right)^{-|\alpha|+1}\left\langle x_{a}\right\rangle^{-\rho}
$$

démonstration et commentaires :

(a) est une conséquence directe du lemme 2.5.

(b) découle des inégalités de Cauchy et des inégalités (2.21).

(c) se déduit des hypothèses $(H)$, des inégalités de Cauchy et de $(2.20)$, pour la première inégalité, et de (2.8) pour la deuxième.

Nous sommes alors en mesure d'estimer les normes des différents opérateurs intervenant dans $\left(I_{1}\right)$.

- Norme de $\sum_{i=2}^{+\infty} \mathcal{P}_{a}\left(Y^{i}\right) \cdot \frac{\left(\nabla^{a}\right)^{i}\left(I_{a}\right)\left(x_{a}\right)}{i !}$, de $E_{\mu}^{1}$ dans lui même .

D'après $(2.28)$ et $(2.29)$ on a pour tout $m_{k} \in E_{k}^{1}$

$$
\left|\mathcal{P}_{a}\left(Y^{i}\right) \frac{\left(\nabla^{a}\right)^{i} I_{a}\left(x_{a}\right)}{i !} m_{k}\right| \leq C^{i} C_{0}^{k+i}(k+i)^{k+i} d\left(x_{a}, \xi_{a}\right)^{-k-i}\left\langle x_{a}\right\rangle^{-\rho}\left\|m_{k}\right\|_{E_{k}^{1}}
$$

Ainsi $\left(\mathcal{P}_{a}\left(Y^{i}\right) \frac{\left(\nabla^{a}\right)^{i} I_{a}\left(x_{a}\right)}{i !}\right) m_{k} \in E_{k+i}^{1}$ et l'application $\left(\mathcal{P}_{a}\left(Y^{i}\right) \frac{\left(\nabla^{a}\right)^{i} I_{a}\left(x_{a}\right)}{i !}\right)$ est un morphisme de $E_{k}^{1}$ dans $E_{k+i}^{1}$ de norme inférieure ou égale à $C^{i}$. C'est donc aussi un endomorphisme de $E_{\mu}^{1}$ de norme majorée par $(\mu C)^{i}$, et la série $\sum_{i=2}^{+\infty} \mathcal{P}_{a}\left(Y^{i}\right) \cdot \frac{\left(\nabla^{a}\right)^{i}\left(I_{a}\right)\left(x_{a}\right)}{i !}$ converge dans $B\left(E_{\mu}^{1}\right)$ pour $\mu<\frac{1}{C}$ et est de norme inférieure ou égale à $\frac{(C \mu)^{2}}{1-\mu C}$. De plus on remarque que son image est incluse dans le sous espace $E_{\mu}^{o}=\left\{\left(m_{k}\right) \in E_{\mu}^{1} \mid m_{0}=0\right\}$.

- Norme de $\sum_{i=1}^{+\infty} \mathcal{P}_{a}\left[\frac{Y^{i} \cdot\left(\nabla^{a}\right)^{i}\left(I_{a}\right)\left(x_{a}\right)}{i !} \times\right.$.] de $E_{\mu}^{2}$ dans $E_{\mu}^{1}$.

En utilisant encore (2.28) et (2.29) il vient, pour tout $m_{k} \otimes P(X, Y) \in E_{k}^{2}$

$$
\mathcal{P}_{a}\left[\frac{Y^{i} \cdot\left(\nabla^{a}\right)^{i} I_{a}\left(x_{a}\right)}{i !}\left(m_{k} \otimes P(X, Y)\right)\right]=m_{k+i}
$$


avec $\left|m_{k+i}\right| \leq C^{i} d\left(x_{a}, \xi_{a}\right)^{-k-i}\left\langle x_{a}\right\rangle^{-\rho}\left\|m_{k}\right\|_{\tilde{E}_{k}^{2}}\left(C_{0}\right)^{i+k}(k+i)^{k+i}\|P(X, Y)\|_{F_{k}}$

$$
\leq C^{i}\left(C_{0}\right)^{i+k}(k+i)^{k+i}\left\langle x_{a}\right\rangle^{-\rho} d\left(x_{a}, \xi_{a}\right)^{-k-i}\left\|m_{k} \otimes P(X, Y)\right\|_{E_{k}^{2}}
$$

ce qui montre que $\mathcal{P}_{a} \frac{(Y)^{i} \cdot\left(\nabla^{a}\right)^{i} I_{a}\left(x_{a}\right)}{i !}$ est un morphisme de $E_{\mu}^{2}$ dans $E_{\mu}^{1}$ de norme inférieure ou égale à $\mu(C \mu)^{i}$. On peut alors comme précédemment donner un sens, pour $\mu<\frac{1}{C}$, à la série $\sum_{i=1}^{+\infty} \mathcal{P}_{a}\left[\frac{Y^{i} \cdot\left(\nabla^{a}\right)^{i}\left(I_{a}\right)\left(x_{a}\right)}{i !} \times\right.$.] qui est un morphisme de $E_{\mu}^{2}$ dans $E_{\mu}^{1}$ de norme inférieure ou égale à $\frac{C \mu^{2}}{1-C \mu}$. Son image est aussi incluse dans $E_{\mu}^{o}$.

- Norme de $\sum_{i=1}^{+\infty} \frac{X Y^{i} \cdot\left(\nabla^{a}\right)^{i}\left(I_{a}\right)\left(x_{a}\right)}{i !}$ de $E_{\mu}^{1}$ dans $E_{\mu}^{2}$.

Avec (2.28) et (2.29) on a pour tout $m_{k} \in E_{k}^{1}$ :

avec

$$
\frac{X Y^{i} \cdot\left(\nabla^{a}\right)^{i}\left(I_{a}\right)\left(x_{a}\right)}{i !} m_{k}=m_{k+i} \otimes\left(X Y^{i}\right)
$$

$$
\left|m_{k+i}\right| \leq C^{i} C_{0}^{k} k^{k} d\left(x_{a}, \xi_{a}\right)^{-k-i}\left\langle x_{a}\right\rangle^{-\rho}\left\|m_{k}\right\|_{E_{k}^{1}}
$$

Ainsi $\left\|m_{k+i}\right\|_{\tilde{E}_{k+i}^{2}} \leq C^{i} C_{0}^{k} k^{k}\left\|m_{k}\right\|_{E_{k}^{1}}$.

Comme d'autre part $\left\|X Y^{i}\right\|_{F_{k+i}} \leq C_{0}^{-k}(k+i)^{-k}$ il vient :

$$
\left\|m_{k+i} \otimes\left(X Y^{i}\right)\right\|_{E_{k+i}^{2}} \leq C^{i}\left\|m_{k}\right\|_{E_{k}^{1}}
$$

ce qui montre que $\frac{X Y^{i} \cdot\left(\nabla^{a}\right)^{i}\left(I_{a}\right)\left(x_{a}\right)}{i !}$ est un opérateur borné de $E_{\mu}^{1}$ dans $E_{\mu}^{2}$ de norme majorée par $C(C \mu)^{i-1}$. En suivant le même raisonnement que précédemment on trouve que, pour $\mu<1 / C, \sum_{i=1}^{+\infty} \frac{X Y^{i} \cdot\left(\nabla^{a}\right)^{i}\left(I_{a}\right)\left(x_{a}\right)}{i !}$ est un morphisme de $E_{\mu}^{1}$ dans $E_{\mu}^{2}$ de norme inférieure ou égale à $C \frac{1}{1-C \mu}$.

- Norme de $\sum_{i=1}^{+\infty} \frac{X Y^{i} \cdot\left(\nabla^{a}\right)^{i}\left(I_{a}\right)\left(x_{a}\right)}{i !}$ de $E_{\mu}^{2}$ dans lui même.

On a pour tout $m_{k} \otimes P(X, Y) \in E_{k}^{2}$

$$
\frac{X Y^{i} \cdot\left(\nabla^{a}\right)^{i}\left(I_{a}\right)\left(x_{a}\right)}{i !} m_{k} \otimes P(X, Y)=m_{k+i} \otimes\left(X Y^{i} P(X, Y)\right)
$$

avec $\left|m_{k+i}\right| \leq C^{i} d\left(x_{a}, \xi_{a}\right)^{(-k-i)}\left\langle x_{a}\right\rangle^{-\rho}\left\|m_{k}\right\|_{\tilde{E}_{k}^{2}}$, ce qui donne donc :

$$
\left\|m_{k+i}\right\|_{\tilde{E}_{k+i}^{2}} \leq C^{i}\left\|m_{k}\right\|_{\tilde{E}_{k}^{2}}
$$

Comme $\left\|X Y^{i} P(X, Y)\right\|_{F_{k+i}} \leq\|P(X, Y)\|_{F_{k}}$ il vient :

$$
\left\|m_{k+i} \otimes\left(X Y^{i} P(X, Y)\right)\right\|_{E_{k+i}^{2}} \leq C^{i}\left\|m_{k} \otimes P(X, Y) \in E_{k}^{2}\right\|_{E_{k}^{2}}
$$

et l'on obtient de façon tout à fait similaire à ce qui précède que, pour $\mu<1 / C, \sum_{i=1}^{+\infty} \frac{X Y^{i} \cdot\left(\nabla^{a}\right)^{i}\left(I_{a}\right)\left(x_{a}\right)}{i !}$ est un endomorphisme de $E_{\mu}^{2}$ de norme inférieure ou égale à $\frac{C \mu}{1-C \mu}$. 
- Norme de $\Delta_{x_{a}}$ de $E_{\mu}^{1}$ dans lui même.

D'après le point (b) du lemme $2.7, \Delta_{x_{a}}$ est un endomorphisme de $E_{\mu}^{1}$ de norme inférieure ou égale à $C_{2} \mu^{2}$ et d'image incluse dans $E_{\mu}^{o}$.

- Norme de $\left(\nabla_{x_{a}} \phi \cdot \nabla_{x_{a}}+i \Delta_{x_{a}} \phi+\Delta_{x_{a}}\right) \otimes X$ de $E_{\mu}^{2}$ dans lui même.

Pour tout $m_{k} \in E_{k}^{2}$ et $P(X, Y) \in F_{k}$ il vient de même en utilisant les résultats du lemme 2.7 :

$$
\begin{gathered}
\left\|\Delta_{x_{a}} m_{k}\right\|_{\tilde{E}_{k+2}^{2}} \leq C_{3}(k+2)^{2}\left\|m_{k}\right\|_{\tilde{E}_{k}^{2}} \\
\left\|\nabla_{a} \phi . \nabla_{a} m_{k}\right\|_{\tilde{E}_{k+1}^{1}} \leq C_{3}\left(\nu+C_{1}\right)(k+1)\left\|m_{k}\right\|_{\tilde{E}_{k}^{2}} \\
\left\|\Delta_{x_{a}} \phi m_{k}\right\|_{\tilde{E}_{k+1}^{1}} \leq C_{1}^{2}\left\|m_{k}\right\|_{\tilde{E}_{k}^{2}} \\
\|X P(X, Y)\|_{F_{k+2}} \leq C_{0}^{-3 / 2}(k+2)^{-2}\|P(X, Y)\|_{F_{k}} \\
\|X P(X, Y)\|_{F_{k+1}} \leq C_{0}^{-1 / 2}(k+1)^{-1}\|P(X, Y)\|_{F_{k}}
\end{gathered}
$$

$\left(\nabla_{x_{a}} \phi \cdot \nabla_{x_{a}}+i \Delta_{x_{a}} \phi+\Delta_{x_{a}}\right) \otimes X$ est donc un endomorphisme de $E_{\mu}^{2}$ de norme majorée par $K \mu(K \in \mathbb{R})$.

- Norme de $\left(R_{0}\right)^{-1}$ de $E_{\mu}^{o}$ dans $E_{\mu}^{1}$.

Il faut tout d'abord construire un inverse à $R_{0}$.

Notons $\rho\left(t, x_{a}, \xi_{a}\right)$ le flot de $\nabla_{a} \phi\left(x_{a}, \xi_{a}\right) . \nabla_{a}$ tel que $\rho\left(0, x_{a}, \xi_{a}\right)=x_{a}$. Gérard et Martinez ont montré dans [G-M] que $\rho\left(t, x_{a}, \xi_{a}\right)$, défini sur $\Gamma^{+}\left(R, d, M, \epsilon_{0}, \sigma^{+}-\delta\right)$, est analytique en $\left(x_{a}, \xi_{a}\right)$ et tel que pour tout $\left(x_{a}, \xi_{a}\right) \in \Gamma, t>0$ :

$$
\rho\left(t, x_{a}, \xi_{a}\right) \in \Gamma_{\xi_{a}} \text { et }\left|\rho\left(t, x_{a}, \xi_{a}\right)\right| \geq \frac{1}{K}\left(\langle\operatorname{Re}(x)\rangle+\left|\xi_{a}\right| t\right)
$$

On sait aussi, d'après (2.19), qu'il existe $\gamma>0$ tel que

$$
d\left(\rho\left(t, x_{a}, \xi_{a}\right), \xi_{a}\right) \geq d\left(x_{a}, \xi_{a}\right)+\gamma t
$$

Pour construire un inverse de $R_{0}$ on commence par définir sur $\Gamma$ :

$$
m_{0}\left(x_{a}, \xi_{a}\right)=\exp \left(-\int_{0}^{+\infty}\left(\Delta_{x_{a}} \phi\left(\rho\left(t, x_{a}, \xi_{a}\right), \xi_{a}\right)-i \lambda^{\alpha} \cdot \nabla^{a} I_{a}\left(\rho\left(t, x_{a}, \xi_{a}\right)\right)\right) d t\right)
$$

dont on vérifie qu'il est analytique en $\left(x_{a}, \xi_{a}\right)$ et tel que :

$$
m_{0}\left(x_{a}, \xi_{a}\right)-1=O\left(\left\langle\operatorname{Re}\left(x_{a}\right)\right\rangle^{-\rho}\right)=O\left(\left\langle x_{a}\right\rangle^{-\rho}\right)
$$


uniformément sur $\Gamma$.

On construit ensuite pour tout élément $m_{k}$ de $\tilde{E}_{k}^{1},(k>0)$, le symbole $m_{k-1}$ par :

$$
m_{k-1}\left(x_{a}, \xi_{a}\right) \otimes \pi_{\alpha}=-i\left(\int_{0}^{+\infty} \frac{m_{k}}{m_{0}}\left(\rho\left(t, x_{a}, \xi_{a}\right), \xi_{a}\right) d t\right) m_{0}\left(x_{a}, \xi_{a}\right) \otimes \pi_{\alpha}
$$

qui est un élément de $\tilde{E}_{k-1}^{1}$ solution de l'équation :

$$
R_{0} m_{k-1}=m_{k}
$$

De plus avec (2.33) et (2.34) il vient :

$$
\begin{aligned}
m_{k-1}\left(x_{a}, \xi_{a}\right) & \leq \int_{0}^{\infty}\left(C_{0} k\right)^{k} d\left(\rho\left(t, x_{a}, \xi_{a}\right), \xi_{a}\right)^{-k}\left\langle\rho\left(t, x_{a}, \xi_{a}\right)\right\rangle^{-\rho}\left\|m_{k}\right\|_{\tilde{E}_{k}^{2}} d t \\
& \leq K_{1} \int_{0}^{+\infty}\left(C_{0} k\right)^{k}\left(d\left(x_{a}, \xi_{a}\right)+\gamma_{1} t\right)^{-k-\rho}\left\|m_{k}\right\|_{\tilde{E}_{k}^{2}} d t \\
& \leq K_{2}\left(C_{0}(k-1)\right)^{(k-1)} d\left(x_{a}, \xi_{a}\right)^{(-k-1)}\left\langle x_{a}\right\rangle^{-\rho}\left\|m_{k}\right\|_{\tilde{E}_{k}^{2}}
\end{aligned}
$$

et $\left\|m_{k-1}\right\|_{E_{k-1}^{1}} \leq K_{2}\left\|m_{k}\right\|_{E_{k}^{1}}$. On a donc construit un inverse de $R_{0}$ de $E_{\mu}^{o}$ dans $E_{\mu}^{1}$ de norme inférieure ou egale à $K_{2} \mu^{-1}$.

Nous venons de passer en revue tous les opérateurs intervenant dans le système $\left(I_{1}\right)$ et d'estimer leur norme. Notons $l_{0}$ la série de $E_{\mu}^{1}$ n'ayant que son premier coefficient non nul, et égal à $\left(m_{0}\right) \otimes \pi_{\alpha}\left(m_{0}\right.$ étant défini par (2.36)), et cherchons une solution $\left(\begin{array}{l}\tilde{m} \\ \tilde{n}\end{array}\right)$ de $I_{1}$ dont le premier terme soit $\left(\begin{array}{l}\left(m_{0}\right) \otimes \pi_{\alpha} \\ 0\end{array}\right)$. Cela s'écrit, en remarquant que $R_{0}\left(m_{0}\right)=0$ sous la forme matricielle suivante :

$$
\left(\begin{array}{c}
\tilde{m} \\
\tilde{n}
\end{array}\right)-\left(\begin{array}{c}
l_{0} \\
0
\end{array}\right)=M\left(\begin{array}{l}
\tilde{m} \\
\tilde{n}
\end{array}\right)
$$

où la matrice $\mathrm{M}$ est de la forme

$$
\left(\begin{array}{rr}
\mu A_{1}^{1} & \mu A_{1}^{2} \\
A_{2}^{1} & \mu A_{2}^{2}
\end{array}\right)
$$

les opérateurs $\left(A_{j}^{i}\right)$ étant, au vu des estimations établies, bornés, uniformément par rapport à $\mu$ proche de zéro. La série $\sum_{j \geq 0} M^{j}$ converge donc dans 
$B\left(E_{\mu}^{1} \times E_{\mu}^{2}\right)$, pour $\mu$ assez petit, et la solution de (2.38) est trivialement donnée par :

$$
\left(\begin{array}{c}
\tilde{m} \\
\tilde{n}
\end{array}\right)=\left(\sum_{j \geq 0} M^{j}\right)\left(\begin{array}{c}
l_{0} \\
0
\end{array}\right) .
$$

La solution que nous venons de construire est une série formelle que nous allons resommer en suivant la méthode développée dans [G-M].

On prend une fonction de troncature $\chi_{0}$, de classe $C^{\infty}(\mathbb{R})$ telle que $X_{0}(x)=0$ si $x \leq 1$ et $\chi_{0}(x)=1$ si $x \geq 2$, et on pose :

$$
p\left(x_{a}, \xi_{a}\right)=\sum_{k=0}^{+\infty}\left(\tilde{m}_{k} \otimes \pi_{\alpha}+\mathcal{P}_{a}\left(\tilde{n}_{k}\right)\right) \chi\left(\frac{\left\langle x_{a}\right\rangle}{C_{1} k}\right)
$$

de telle sorte que pour $\left(x_{a}, \xi_{a}\right)$ fixés la somme ne porte que sur un nombre fini d'indices. De plus en utilisant le lemme 2.5 il vient :

$$
\left\|e^{\epsilon_{0}\left\langle x^{a}\right\rangle}\left(\tilde{m}_{k} \otimes \pi_{\alpha}+\mathcal{P}_{a}\left(\tilde{n}_{k}\right)\right)\right\|_{L^{2}\left(X^{a}\right)} \leq C^{k} k^{k}\left\langle x_{a}\right\rangle^{-k}
$$

Posons :

$$
p^{\prime}\left(x_{a}, \xi_{a}\right)=e^{\epsilon_{0}\left\langle x^{a}\right\rangle} p\left(x_{a}, \xi_{a}\right)
$$

En choisissant $C_{1}>C$ les symboles $\tilde{n}_{k}$ et $\tilde{m}_{k}$ étant analytiques dans $\Omega$ on a les estimations :

$$
\left\|\frac{\partial p^{\prime}}{\partial \bar{x}_{a}}\right\|_{L^{2}\left(X^{a}\right)}+\left\|\frac{\partial p^{\prime}}{\partial \bar{\xi}_{a}}\right\|_{L^{2}\left(X^{a}\right)} \leq e^{-\epsilon_{2}\left\langle x_{a}\right\rangle}
$$

uniformément sur $\Omega$. Utilisons alors le théorème 3.2 de [L] que l'on rappelle ici :

Théorème 2.8 - Soit $\omega$ un ouvert borné de $\mathbb{R}^{n}$ et $U$ un ouvert quasiconique de $\mathbb{C}^{n} \times \mathbb{C}^{n}$ de base $\omega \times \mathbb{R}^{n}$. Alors pour tout $f \in C^{\infty}(U)$ telle que $\bar{\partial} f$ soit exponentiellement décroissante, il existe un ouvert quasi-conique $V \subset U$, de base $\omega \times \mathbb{R}^{n}$ et pseudo-convexe ainsi qu'une fonction $g \in C^{\infty}(V)$ exponentiellement décroissante telle que $\bar{\partial} f=\bar{\partial} g$ dans $V$. (avec la notation $\left.\bar{\partial} f=\left(\frac{\partial f}{\partial \bar{x}_{a}}, \frac{\partial f}{\partial \bar{\xi}_{a}}\right)\right)$

En application directe on en déduit l'existence d'un fonction $q\left(x_{a}, \xi_{a}\right)$ de classe $C^{\infty}$ sur $\Gamma^{\prime+}=\Gamma^{+}\left(R^{\prime}, d, M, \epsilon^{\prime}, \sigma^{+}\right)$telle que :

$$
q\left(x_{a}, \xi_{a}\right)=O\left(e^{-\epsilon_{2}\left\langle x_{a}\right\rangle}\right) \text { et } \bar{\partial} p^{\prime}=\bar{\partial} q \text { sur } \Gamma^{\prime+}
$$

En posant $m\left(x_{a}, \xi_{a}\right)=e^{-\epsilon_{0}\left\langle x^{a}\right\rangle}\left(p^{\prime}-q\right)\left(x_{a}, \xi_{a}\right)$ on obtient une solution à la proposition 2.4 sur $\Gamma^{+}\left(R^{\prime}, d, M, \epsilon^{\prime}, \sigma^{+}\right)$. 


\subsection{Construction des opérateurs d'onde pour un canal de réaction quelconque.}

Une des propriétés essentielles qui nous a permis d'effectuer notre construction des opérateurs d'onde est que dans le cas où $a$ est une décomposition en deux amas, le terme d'interaction $I_{a}\left(x_{a}\right)$ a la structure d'un potentiel à deux corps. Pour généraliser notre construction au cas où \#a>2 l'idée relativement simple consiste seulement à dire que si $x_{a}$ reste dans une région conique ne comportant pas les plans de collision, c'est à dire dans la région où nous étudions la diffusion, $I_{a}\left(x_{a}\right)$ a encore la structure d'un potentiel à deux corps.

Pour généraliser la construction d'Isozaki-Kitada, comme nous l'avons fait en introduction, les hypothèses d'analyticité sur les potentiels sont en fait excessives, des hypothèses de régularité et de décroissance similaires à celle de [I-K] suffisant. C'est ainsi que nous avons pu traiter à la fois les cas $\# a=2$ et $\# a>2$, en utilisant dans ce dernier cas le terme d'interaction $\tilde{I}_{a}(x)$ au lieu de $I_{a}(x)$. (Cf définition 1.4). En revanche pour la nouvelle construction que nous venons d'exposer ci dessus les hypothèses d'analyticité faites sur les potentiels sont essentielles et ne sont pas satisfaites par $\tilde{I}_{a}(x)$ qui comporte des fonctions de troncature. Cependant nous avons vu qu'avec la définition des opérateurs d'onde :

$$
W_{\alpha}^{ \pm}=s-\lim _{t \rightarrow \pm \infty} e^{i t H} J_{a} e^{-i t H_{a}} \chi_{a_{0}}\left(\frac{D_{x_{a}}}{\left|D_{x_{a}}\right|}\right) p_{\alpha}
$$

nous avons aussi :

$$
W_{\alpha}^{ \pm}=s-\lim _{t \rightarrow \pm \infty} e^{i t H} \chi_{a_{1}}\left(\frac{x_{a}}{\left|x_{a}\right|}\right) J_{a} e^{-i t H_{a}} \chi_{a_{0}}\left(\frac{D_{x_{a}}}{\left|D_{x_{a}}\right|}\right) p_{\alpha}
$$

de telle sorte que nous aurons besoin de construire nos fonctions de phase $\phi_{a, j}\left(x_{a}, \xi_{a}\right)$ et nos symboles $m_{a, j}\left(x_{a}, \xi_{a}\right)$ que pour $\xi_{a} \in \operatorname{supp} \chi_{a_{0}}\left(\frac{\xi_{a}}{\left|\xi_{a}\right|}\right)$ et pour $x_{a} \in \operatorname{supp}\left(\chi_{a_{1}}\left(\frac{x_{a}}{\left|x_{a}\right|}\right)\right)$ c'est à dire dans la région où $\tilde{I}_{a}(x)$ est analytique.

Pour éviter de réécrire entièrement la construction des fonctions de phase et des symboles, nous pouvons nous contenter de constater qu'avec la définition 1.2 :

$$
\left.\begin{array}{c}
\xi_{a} \in \operatorname{supp} \chi_{a_{0}} \\
\text { et } \cos \left(x_{a}, \xi_{a}\right) \geq \tilde{\sigma}_{K_{a}}
\end{array}\right\} \Rightarrow \chi_{a_{2}}\left(\frac{x_{a}}{\left|x_{a}\right|}\right)=1
$$

de telle sorte que $\tilde{I}_{a}(x)$ est holomorphe dans la région $\Gamma_{a}^{+}\left(R, d, M, \epsilon, \tilde{\sigma}_{K_{a}}\right)$, et l'on peut reprendre sans modification les résultats des propositions 2.3 et 2.4 en nous limitant à la région sortante et au cas $\sigma_{j}^{+}>\tilde{\sigma}_{K_{a}}$. 
Notre travail ne traitant que de la diffusion 2 amas - $k$ amas nous n'utilisons, pour les décompositions à plus de deux amas, que l'opérateur d'onde sortant. Nous définirons conventionnellement $\phi_{a}^{-}\left(x_{a}, \xi_{a}\right)=0$ et $m_{j}^{-}\left(x_{a}, \xi_{a}\right)=0$.

Le théorème 2.2 peut être alors repris pour les canaux de réaction à plus de 2 amas, avec pour seules modifications le fait que nous nous limiterons à $\sigma_{j}^{+}>\tilde{\sigma}_{K_{a}}$ et que le point (3) du théorème n'est alors vrai que pour l'opérateur d'onde sortant $W_{\alpha}^{+}$. (L'opérateur d'onde entrant ayant été pris conventionnellement égal à zéro. )

Nous n'oublierons pas non plus que ce qui distingue les propriétés des modificateurs, entre le cas $\# a=2$ et $\# a>2$, s'exprime aussi par le fait que dans le cas \# $a=2$ les fonctions $\chi_{a_{0}}$ et $\chi_{a_{1}}$ sont identiquement égales à 1 , alors que dans le cas \# $a>2$ ces fonctions sont nulles près des plans de collision. En particulier dans ce dernier cas le commutateur $\left[D_{x_{a}}^{2}, \chi_{a_{1}}\left(\frac{x_{a}}{\mid x_{a}}\right)\right]$ n'est pas nul, et nous devrons le prendre en compte tout au long de notre raisonnement. 



\section{Démonstration du théorème $\mathbf{1 . 9}$}

Les opérateurs d'onde que nous venons de construire dans les sections précédentes ont des propriétés similaires à ceux de Gérard et Martinez, de telle sorte que la démarche que nous adoptons pour démontrer le théorème 1.9 va pouvoir suivre en grande partie celle de [G-M]. Seuls quelques arguments nouveaux doivent intervenir dans le cas où les amas d'avant et après diffusion sont différents, et en particulier dans le cas où le canal de sortie n'est pas à deux amas.

A la base de la démonstration se trouve une formule de représentation de la matrice de diffusion analogue à celle introduite par Isozaki et Kitada pour le problème à deux corps dans [I-K], que nous exposons ci dessous.

Théorème 3.1 Sous les hypothèses $(H)$ et $(E)$, on a pour tout $\lambda \in[d, M]$ :

$$
\begin{aligned}
\mathcal{S}_{\alpha \beta}(\lambda)= & p_{\alpha}^{*} \delta_{\alpha, \beta} p_{\alpha}+2 i \pi \lim _{\epsilon^{\prime} \rightarrow 0^{+}} \mathcal{F}_{\beta}(\lambda) p_{\beta}^{*} T_{b, 2}^{*} R\left(\lambda+i \epsilon^{\prime}\right) T_{a, 1} p_{\alpha} \mathcal{F}_{\alpha}(\lambda)^{*} \\
& -2 i \pi \mathcal{F}_{\beta}(\lambda) p_{\beta}^{*} J_{b, 2}^{*} T_{a, 1} p_{\alpha} \mathcal{F}_{\alpha}(\lambda)^{*}
\end{aligned}
$$

Démonstration du théorème 3.1 :

La difficulté principale pour obtenir ce résultat consiste en fait à montrer que la limite écrite ci-dessus a effectivement un sens. Cela peut être fait dans un cadre plus général à l'aide d'estimations sur les valeurs au bord de la résolvante obtenues par la théorie de Mourre (comme dans [Bo2]), mais dans le cas présent il s'avère bien plus simple d'étudier cette limite après avoir effectué une distorsion analytique, le problème, nous le verrons devenant 
alors trivial. Comme nous ne faisons intervenir les distorsions analytiques que dans la section suivante nous laisserons ce point en suspens quelques temps, avant de le traiter rigoureusement dans le paragraphe 3.2.

Nous présentons cependant ici le reste de la démonstration qui suit une démarche que l'on trouve dans [I-K].

On calcule pour commencer :

$$
\begin{gathered}
\left(W_{\alpha}^{+}\right)^{*}\left(W_{\beta}^{+}\right)=s-\lim _{t \rightarrow+\infty} p_{\alpha}^{*} e^{i t H_{a}} J_{a, 1}^{*} J_{b, 2} e^{-i t H_{b}} p_{\beta} \\
=\delta_{\alpha, \beta} p_{\alpha}^{*} p_{\alpha}
\end{gathered}
$$

car les images de $W_{\alpha}^{+}$et $W_{\beta}^{+}$sont orthogonales pour $\alpha \neq \beta$ et car $\left(I-J_{a, 1}^{*} J_{a, 1}\right)$ est compact dans $L^{2}\left(X_{a}\right)$. Ainsi :

$$
S_{\alpha, \beta}(\Delta)=\left(W_{\beta}^{+}\right)^{*}\left(W_{\alpha}^{-}-W_{\alpha}^{+}\right)+\delta_{\alpha, \beta} p_{\alpha}^{*} p_{\alpha}
$$

On notera : $\tilde{S}_{\alpha, \beta}(\Delta)=S_{\alpha, \beta}(\Delta)-\delta_{\alpha, \beta} p_{\alpha}^{*} p_{\alpha}$

Et $\forall f \in \mathcal{S}\left(\mathbb{R}^{n}\right), \forall g \in \mathcal{S}\left(\mathbb{R}^{n}\right) \quad$ avec la notation $\quad T_{a, 1}=H J_{a, 1}-J_{a, 1} H_{a}$ on a :

$$
\begin{aligned}
\left(\tilde{S}_{\alpha, \beta}(\Delta) f, g\right)= & \left(\left(W_{\alpha}^{-}-W_{\alpha}^{+}\right) f, W_{\beta}^{+} g\right) \\
= & -i \int_{-\infty}^{+\infty}\left(e^{i t H} T_{a, 1} e^{-i t H_{a}} p_{\alpha} f, W_{\beta}^{+} g\right) d t \\
= & -\int_{-\infty}^{+\infty} d t \int_{0}^{+\infty} d \sigma\left(T_{a, 1} e^{-i t H_{a}} p_{\alpha} f, e^{i \sigma H} T_{b, 2} e^{-i(\sigma+t) H_{b}} p_{\beta} g\right) \\
& -i \int_{-\infty}^{+\infty} d t\left(T_{a, 1} e^{-i t H_{a}} p_{\alpha} f, J_{b, 2} e^{-i t H_{b}} p_{\beta} g\right) \\
= & -\int_{-\infty}^{+\infty} d t \int_{0}^{+\infty} d \sigma\left(e^{i(\sigma+t) H_{b}} T_{b, 2}^{*} e^{-i \sigma H} T_{a, 1} e^{-i t H_{a}} p_{\alpha} f, p_{\beta} g\right) \\
& -i \int_{-\infty}^{+\infty} d t\left(e^{i t H_{b}} J_{b, 2}^{*} T_{a, 1} e^{-i t H_{a}} p_{\alpha} f, p_{\beta} g\right)
\end{aligned}
$$

Appelons $I_{1}$ et $I_{2}$ les deux intégrales se trouvant dans le dernier terme de cette égalité. En faisant intervenir les opérateurs transformée de Fourier $\mathcal{F}_{\alpha}$ et $\mathcal{F}_{\beta}$ définis dans l'introduction, en utilisant (1.3) et (1.4) on obtient :

$$
\begin{aligned}
I_{1}= & \lim _{\epsilon, \epsilon^{\prime} \rightarrow 0^{+}} \int_{0}^{+\infty} d \sigma \int_{-\infty}^{+\infty} d t \int_{\epsilon_{\beta}}^{+\infty} d \lambda \\
& \left(\left(\mathcal{F}_{\beta} p_{\beta}^{*} T_{b, 2}^{*} e^{-i \sigma(H-\lambda-i \epsilon)} T_{a, 1} e^{-i t\left(H_{a}-\lambda\right)-\epsilon^{\prime}|t|} p_{\alpha} f\right)(\lambda, .), \mathcal{F}_{\beta}(\lambda) g\right)_{L^{2}\left(S_{b}^{n-1}\right)} \\
= & \lim _{\epsilon, \epsilon^{\prime} \rightarrow 0^{+}} \int_{-\infty}^{+\infty} d t \int_{\epsilon_{\beta}}^{+\infty} d \lambda \\
& \left(\left(\mathcal{F}_{\beta} p_{\beta}^{*} T_{b, 2}^{*} R(\lambda+i \epsilon) T_{a, 1} e^{-i t\left(H_{a}-\lambda\right)-\epsilon^{\prime}|t|} p_{\alpha} f\right)(\lambda, .), \mathcal{F}_{\beta}(\lambda) g\right)_{L^{2}\left(S_{b}^{n-1}\right)}
\end{aligned}
$$

Introduisons alors $\mathcal{F}_{\alpha}^{*} \mathcal{F}_{\alpha}=1$ dans chacune des intégrales $I_{1}$ et $I_{2}$. Il vient : 


$$
\begin{aligned}
& I_{1}=\lim _{\epsilon, \epsilon^{\prime} \rightarrow 0^{+}}-2 i \pi \int_{-\infty}^{+\infty} d t \int_{\epsilon_{\beta}}^{+\infty} d \lambda \int_{\epsilon_{\alpha}}^{+\infty} d \lambda^{\prime} \\
& e^{i t\left(\lambda-\lambda^{\prime}\right)-\epsilon^{\prime}|t|}\left(\left(\mathcal{F}_{\beta} p_{\beta}^{*} T_{b, 2}^{*} R(\lambda+i \epsilon) T_{a, 1} p_{\alpha} \mathcal{F}_{\alpha}\left(\lambda^{\prime}\right)^{*} \mathcal{F}_{\alpha}\left(\lambda^{\prime}\right) f\right)(\lambda, .), \mathcal{F}_{\beta}(\lambda) g\right)_{L^{2}\left(S_{b}^{n-1}\right)}
\end{aligned}
$$

et :

$$
\begin{aligned}
I_{2}= & \lim _{\epsilon^{\prime} \rightarrow 0^{+}} 2 \pi \int_{-\infty}^{+\infty} d t \int_{\epsilon_{\beta}}^{+\infty} d \lambda \int_{\epsilon_{\alpha}}^{+\infty} d \lambda^{\prime} \\
& e^{i t\left(\lambda-\lambda^{\prime}\right)-\epsilon^{\prime}|t|}\left(\left(\mathcal{F}_{\beta} p_{\beta}^{*} J_{b, 2}^{*} T_{a, j} p_{\alpha} \mathcal{F}_{\alpha}\left(\lambda^{\prime}\right)^{*} \mathcal{F}_{\alpha}\left(\lambda^{\prime}\right) f\right)(\lambda, .), \mathcal{F}_{\beta}(\lambda) g\right)_{L^{2}\left(S_{b}^{n-1}\right)}
\end{aligned}
$$

Ce qui donne pour finir :

$$
\begin{aligned}
\left(\tilde{S}_{\alpha, \beta}(\Delta) f, g\right) & =2 i \pi \int_{s u p\left(\epsilon_{\alpha}, \epsilon_{\beta}\right)}^{+\infty} d \lambda \\
{\left[\lim _{\epsilon \rightarrow 0^{+}}(\right.} & \left.\left(\mathcal{F}_{\beta} p_{\beta}^{*} T_{b, 2}^{*} R(\lambda+i \epsilon) T_{a, 1} p_{\alpha} \mathcal{F}_{\alpha}(\lambda)^{*} \mathcal{F}_{\alpha}(\lambda) f\right)(\lambda, .), \mathcal{F}_{\beta}(\lambda) g\right)_{L^{2}\left(S_{b}^{n-1}\right)} \\
& \left.\quad\left(\left(\mathcal{F}_{\beta} p_{\beta}^{*} J_{b, 2}^{*} T_{a, j} p_{\alpha} \mathcal{F}_{\alpha}(\lambda)^{*} \mathcal{F}_{\alpha}(\lambda) f\right)(\lambda, .), \mathcal{F}_{\beta}(\lambda) g\right)_{L^{2}\left(S_{b}^{n-1}\right)}\right]
\end{aligned}
$$

ce qui n'est rien d'autre que le résultat annoncé. (L'existence de la limite écrite ci-dessus sera on le rappelle justifiée au paragraphe 3.2)

Ecrivons encore la formule de représentation de la matrice de diffusion que nous venons d'établir ainsi :

$$
S_{\alpha, \beta}\left(\omega_{a}, \theta_{b}, \lambda\right)=\delta_{\alpha, \beta} \delta\left(\theta_{b}-\omega_{a}\right)+4 i \pi\left(\left(\lambda-\epsilon_{\alpha}\right)\left(\lambda-\epsilon_{\beta}\right)\right)^{\left(\frac{n-2}{4}\right)}\left(M_{2}-M_{1}\right)
$$

avec :

$$
\begin{gathered}
M_{1}=\left(e^{i \sqrt{\lambda-\epsilon_{\beta}} \theta_{b} \cdot x_{b}} \otimes \psi_{\beta}\left(x^{b}\right), J_{b, 2}^{*} T_{a, 1} e^{i \sqrt{\lambda-\epsilon_{\alpha}} \omega_{a} \cdot x_{a}} \otimes \psi_{\alpha}\left(x^{a}\right)\right) \\
M_{2}=\lim _{\epsilon^{\prime} \rightarrow 0^{+}} M_{2}\left(\epsilon^{\prime}\right) \\
M_{2}\left(\epsilon^{\prime}\right)=\left(e^{i \sqrt{\lambda-\epsilon_{\beta}} \theta_{b} \cdot x_{b}} \otimes \psi_{\beta}\left(x^{b}\right), T_{b, 2}^{*} R\left(\lambda+i \epsilon^{\prime}\right) T_{a, 1} e^{i \sqrt{\lambda-\epsilon_{\alpha}} \omega_{a} \cdot x_{a}} \otimes \psi_{\alpha}\left(x^{a}\right)\right)
\end{gathered}
$$

expressions qui peuvent encore s'écrire en remplaçant les opérateurs $T_{a, j}$ et $J_{a, j}$ par leur forme intégrale :

$$
\begin{gathered}
M_{1}=\left(w_{b}(x) \chi_{b_{1}}\left(\frac{x_{b}}{\left|x_{b}\right|}\right) e^{i \phi_{b, 2}\left(x_{b}, \sqrt{\lambda-\epsilon_{\beta}} \theta_{b}\right)} m_{b, 2}\left(x_{b}, \sqrt{\lambda-\epsilon_{\beta}} \theta_{b}\right) \psi_{\beta}\left(x^{b}\right), s_{a, 1}(x)\right)_{L^{2}(X)} \\
M_{2}\left(\epsilon^{\prime}\right)=\left(s_{b, 2}(x), R\left(\lambda+i \epsilon^{\prime}\right) s_{a, 1}(x)\right)_{L^{2}(X)}
\end{gathered}
$$


où :

$$
\begin{aligned}
s_{a, 1}(x)= & w_{a}(x) \chi_{a_{1}}\left(\frac{x_{a}}{\left|x_{a}\right|}\right) e^{i \phi_{a, 1}\left(x_{a}, \sqrt{\lambda-\epsilon_{\alpha}} \omega_{a}\right)} t_{a, 1}\left(x_{a}, \sqrt{\lambda-\epsilon_{\alpha}} \omega_{a}\right) \psi_{\alpha}\left(x^{a}\right) \\
& +\left[D_{x}^{2}, w_{a}(x) \chi_{a_{1}}\left(\frac{x_{a}}{\left|x_{a}\right|}\right)\right] e^{i \phi_{a, 1}\left(x_{a}, \sqrt{\lambda-\epsilon_{\alpha}} \omega_{a}\right)} m_{a, 1}\left(x_{a}, \sqrt{\lambda-\epsilon_{\alpha}} \omega_{a}\right) \psi_{\alpha}\left(x^{a}\right)
\end{aligned}
$$

et

$$
\begin{aligned}
s_{b, 2}(x)= & w_{b}(x) \chi_{b_{1}}\left(\frac{x_{b}}{\left|x_{b}\right|}\right) e^{i \phi_{b, 2}\left(x_{b}, \sqrt{\lambda-\epsilon_{\beta}} \theta_{b}\right)} t_{b, 2}\left(x_{b}, \sqrt{\lambda-\epsilon_{\beta}} \theta_{b}\right) \psi_{\beta}\left(x^{b}\right) \\
& +\left[D_{x}^{2}, w_{b}(x) \chi_{b_{1}}\left(\frac{x_{b}}{\mid x_{b}}\right)\right] e^{i \phi_{b, 2}\left(x_{b}, \sqrt{\lambda-\epsilon_{\beta}} \theta_{b}\right)} m_{b, 2}\left(x_{b}, \sqrt{\lambda-\epsilon_{\beta}} \theta_{b}\right) \psi_{\beta}\left(x^{b}\right)
\end{aligned}
$$

Rappelons ici que les termes $M_{1}$ et $M_{2}\left(\epsilon^{\prime}\right)$ sont définis a priori comme des inégrales oscillantes.

Dans toute la suite nous allons supposer que les constantes $\sigma_{j}^{ \pm}$qui interviennnent dans le théorème 2.2 ont été choisies de telle sorte que :

$$
\left\{\begin{array}{ccc}
-1<\sigma_{2}^{-}<\sigma_{2}^{+}<0<\sigma_{1}^{-}<\sigma_{1}^{+}<1 & \text { si } & \# b=2 \\
\tilde{\sigma}_{K_{b}}<\sigma_{2}^{-}<\sigma_{2}^{+}<\sigma_{K_{b}}<\sigma_{1}^{-}<\sigma_{1}^{+}<1 & \text { si } & \# b>2
\end{array}\right.
$$

Rappelons que la valeur des opérateurs d'onde est indépendante de ces choix.

Nous allons prouver alors que pour tout $\lambda^{\prime} \in[d, M]$ chacun des termes $M_{1}$ et $M_{2}$ se prolonge méromorphiquement en $\lambda$ sur un voisinage de $\lambda^{\prime}$ de la forme :

$$
\mathcal{D}_{\lambda^{\prime}, \epsilon}=\left\{\lambda|| \operatorname{Re}(\lambda)-\lambda^{\prime}|<\epsilon,| \operatorname{Im}(\lambda) \mid \leq \epsilon\right\}
$$

Nous supposerons pour la suite que toutes les fonctions de troncature que nous avons intoduites dans la construction des opérateurs sont Gevrey $s$, avec $s>1$.

\subsection{Prolongement de $M_{1}$}

Nous distinguerons deux cas :

- Le cas $a=b$, c'est à dire lorsque les amas en présence avant et après diffusion sont identiques.

- Le cas $a \neq b$.

- Cas où $a=b$ 
Rappelons que comme ici $a$ et $b$ sont toutes deux des décompositions en deux amas nous avons $\chi_{a_{1}}=\chi_{b_{1}}=1$.

De par leur définition les symboles $m_{a, j}\left(x_{a}, \xi_{a}\right)$ sont nuls dans les régions $\cos \left(x_{a}, \xi_{a}\right) \in\left[\sigma_{j}^{-}-\delta, \sigma_{j}^{+}+\delta\right]$, de telle sorte que là où le produit $m_{a, 2}\left(x_{a}, \xi_{a}\right) t_{a, 1}\left(x_{a}, \xi_{a}\right)$ n'est pas nul les fonctions de phase $\phi_{a, j}\left(x_{a}, \xi_{a}\right)$ sont égales à une des fonctions $\Phi^{ \pm}\left(x_{a}, \xi_{a}\right)$ données par la proposition 2.3 qui sont holomorphes et vérifient :

$$
\Phi^{ \pm}\left(x_{a}, \xi_{a}\right)=x_{a} \cdot \xi_{a}+O\left(\left\langle x_{a}\right\rangle^{1-\rho}\right)
$$

uniformément dans $\Gamma_{a}^{ \pm}(R, d, M, \epsilon, \pm \sigma)$.

On peut donc écrire :

$$
\begin{aligned}
M_{1}=\int_{X_{a}} & e^{i x_{a}\left(\sqrt{\lambda-\epsilon_{\alpha}} \omega_{a}-\sqrt{\lambda-\epsilon_{\beta}} \theta_{a}\right)+r\left(\lambda, x_{a}, \omega_{a}, \theta_{a}\right)} \\
& \times\left[\left(w_{a}(x) m_{a, 2}\left(x_{a}, \sqrt{\lambda-\epsilon_{\beta}} \theta_{a}\right) \psi_{\beta}\left(x^{a}\right),\right.\right. \\
& \left.w_{a}(x) t_{a, 1}\left(x_{a}, \sqrt{\lambda-\epsilon_{\alpha}} \omega_{a}\right) \psi_{\alpha}\left(x^{a}\right)\right)_{L^{2}\left(X^{a}\right)} \\
& +\left(w_{a}(x) m_{a, 2}\left(x_{a}, \sqrt{\lambda-\epsilon_{\beta}} \theta_{a}\right) \psi_{\beta}\left(x^{a}\right),\right. \\
& {\left.\left.\left[D_{x}^{2}, w_{a}(x)\right] m_{a, 1}\left(x_{a}, \sqrt{\lambda-\epsilon_{\alpha}} \omega_{a}\right) \psi_{\alpha}\left(x^{a}\right)\right)_{L^{2}\left(X^{a}\right)}\right] d x_{a} }
\end{aligned}
$$

où $r\left(\lambda, x_{a}, \omega_{a}, \theta_{a}\right)$ est une fonction holomorphe sur un ouvert contenant les support des produits $m_{a, 2}() t_{a, 1}()$ et $m_{a, 2}() m_{a, 1}()$, et de taille $O\left(\left\langle x_{a}\right\rangle^{1-\rho}\right)$ uniformément par rapport à $\lambda, \omega_{a}, \theta_{a}$.

Séparons maintenant l'intégrale (3.7) en quatre termes $M_{1}=L_{0}+L_{1}+$ $L_{2}+L_{3}$ avec :

$$
\begin{gathered}
L_{0}=\int_{X_{a}} e^{i x_{a}\left(\sqrt{\lambda-\epsilon_{\alpha}} \omega_{a}-\sqrt{\lambda-\epsilon_{\beta}} \theta_{a}\right)} l_{0}\left(\lambda, x_{a}, \omega_{a}, \theta_{a}\right) d x_{a} \\
L_{1}=\int_{\left|x_{a}\right| \leq R} e^{i x_{a}\left(\sqrt{\lambda-\epsilon_{\alpha}} \omega_{a}-\sqrt{\lambda-\epsilon_{\beta}} \theta_{a}\right)} l_{1}\left(\lambda, x_{a}, \omega_{a}, \theta_{a}\right) d x_{a} \\
L_{k}=\int_{\left|x_{a}\right| \geq R} e^{i x_{a}\left(\sqrt{\lambda-\epsilon_{\alpha}} \omega_{a}-\sqrt{\lambda-\epsilon_{\beta}} \theta_{a}\right)} l_{k}\left(\lambda, x_{a}, \omega_{a}, \theta_{a}\right) d x_{a} \quad(k=2,3)
\end{gathered}
$$

les symboles $l_{i}\left(\lambda, x_{a}, \omega_{a}, \theta_{a}\right)$ étant définis par :

$$
\begin{aligned}
l_{0}\left(\lambda, x_{a}, \omega_{a}, \theta_{a}\right)= & e^{r\left(\lambda, x_{a}, \omega_{a}, \theta_{a}\right)} \times\left(w_{a}(x) m_{a, 2}\left(x_{a}, \sqrt{\lambda-\epsilon_{\beta}} \theta_{a}\right) \psi_{\beta}\left(x^{a}\right),\right. \\
& {\left.\left[D_{x}^{2}, w_{a}(x)\right] m_{a, 1}\left(x_{a}, \sqrt{\lambda-\epsilon_{\beta}} \theta_{a}\right) \psi_{\alpha}\left(x^{a}\right)\right)_{L^{2}\left(X^{a}\right)} } \\
l_{1}\left(\lambda, x_{a}, \omega_{a}, \theta_{a}\right)= & e^{r\left(\lambda, x_{a}, \omega_{a}, \theta_{a}\right)} \times\left(w_{a}(x) m_{a, 2}\left(x_{a}, \sqrt{\lambda-\epsilon_{\beta}} \theta_{a}\right) \psi_{\beta}\left(x^{a}\right),\right. \\
& \left.w_{a}(x) t_{a, 1}\left(x_{a}, \sqrt{\lambda-\epsilon_{\alpha}} \omega_{a}\right) \psi_{\alpha}\left(x^{a}\right)\right)_{L^{2}\left(X^{a}\right)} \\
l_{2}\left(\lambda, x_{a}, \omega_{a}, \theta_{a}\right)= & l_{1}\left(\lambda, x_{a}, \omega_{a}, \theta_{a}\right)(1-\chi)\left(\cos \left(x_{a}, \omega_{a}\right)\right) \\
l_{3}\left(\lambda, x_{a}, \omega_{a}, \theta_{a}\right)= & l_{1}\left(\lambda, x_{a}, \omega_{a}, \theta_{a}\right) \chi\left(\cos \left(x_{a}, \omega_{a}\right)\right)
\end{aligned}
$$


la fonction de troncature $\chi$ étant Gevrey $s$, égale à 1 sur $\left[\sigma_{1}^{-}, \sigma_{1}^{+}\right]$et de support inclus dans $\left[\sigma_{1}^{-}-\delta, \sigma_{1}^{+}+\delta\right]$.

Examinons chacun de ces termes :

$\underline{L_{0}}$ : Utilisant les propriétés de support des dérivées de la fonction de troncature $w_{a}(x)$, supportées dans des région du type $\left\langle x_{a}\right\rangle \simeq\left\langle x^{a}\right\rangle$, et le fait que les opérateurs $e^{\epsilon_{0}\left\langle x^{a}\right\rangle} m_{a, 1}\left(x_{a}, \xi_{a}\right)$ et $e^{\epsilon_{0}\left\langle x^{a}\right\rangle} D_{x^{a}} m_{a, 1}\left(x_{a}, \xi_{a}\right)$ sont bornés, uniformément par rapport à $x_{a}$, on constate sans difficultés que $l_{0}\left(\lambda, x_{a}, \omega_{a}, \theta_{a}\right)$, qui est un terme Gevrey $s$ en $\omega_{a}, \theta_{a}$, holomorphe en $\lambda$ sur un domaine de type $\mathcal{D}_{\lambda^{\prime}, \epsilon}$ est exponentiellement décroissant en $\left\langle x_{a}\right\rangle$. Il en suit que pour $\epsilon<<\epsilon_{0}$, $L_{0}$, qui est alors une intégrale absolument convergente, définit une fonction de $G^{s}\left(S_{a} \times S_{b}\right)$ holomorphe en $\lambda \in \mathcal{D}_{\lambda^{\prime}, \epsilon}$.

$L_{1}$ : L'intégrale définissant $L_{1}$ portant sur un domaine borné on constate aisément, au vu du théorème 2.2, que le terme $L_{1}$ se prolonge holomorphiquement en $\lambda$ comme une fonction $G^{s}\left(S_{a} \times S_{a}\right)$, sur un domaine $\mathcal{D}_{\lambda^{\prime}, \epsilon^{\prime}}$. $\underline{L_{2}}$ : Sur le support de $(1-\chi)$ le symbole $t_{a, 1}\left(x_{a}, \sqrt{\lambda-\epsilon_{\alpha}} \omega_{a}\right)$ est exponentiellement décroissant en $\left\langle x_{a}\right\rangle$ d'après le Théorème 2.2. Ainsi les fonctions $\partial_{\omega_{a}}^{\alpha} \partial_{\theta_{a}}^{\beta} l_{2}\left(\lambda, x_{a}, \omega_{a}, \theta_{a}\right)$, qui sont holomorphes en $\lambda$ sur un domaine $\mathcal{D}_{\lambda^{\prime}, \epsilon}$, vérifient les estimations :

$$
\left|\partial_{\omega_{a}}^{\alpha} \partial_{\theta_{a}}^{\beta} l_{2}\left(\lambda, x_{a}, \omega_{a}, \theta_{a}\right)\right| \leq C^{1+|\alpha|+|\beta|} e^{-\epsilon_{1}\left\langle x_{a}\right\rangle}(|\alpha| !)^{s}(|\beta| !)^{s}
$$

uniformément en $\lambda$, pour $\epsilon_{1}<\epsilon_{0}$. L'intégrale $L_{2}$ est donc absolument convergente, donnant un terme $G^{s}\left(S_{a} \times S_{a}\right)$ holomorphe en $\lambda$ sur un domaine $\mathcal{D}_{\lambda^{\prime}, \epsilon}$.

$\underline{L_{3}}$ : Remarquons que de par la présence des fonctions $w_{a}(x)$ dans la définiton de $l_{1}$, le terme $l_{3}$ n'est pas holomorphe en $\left|x_{a}\right|$ dans la région $\left|x_{a}\right|>R$. Pour pallier à cet inconvénient nous introduisons de part et d'autre dans le produit scalaire définissant $l_{1}$ l'identité $w_{a}(x)=1+\left(w_{a}(x)-1\right)$, de telle 
sorte que $l_{3}$ se décompose en quatre termes, $l_{3}=l_{3}^{1}+l_{3}^{2}+l_{3}^{3}+l_{3}^{4}$, avec :

$$
\begin{aligned}
l_{3}^{1}\left(\lambda, x_{a}, \omega_{a}, \theta_{a}\right)= & \chi\left(\cos \left(x_{a}, \omega_{a}\right)\right) e^{r\left(\lambda, x_{a}, \omega_{a}, \theta_{a}\right)} \times\left(m_{a, 2}\left(x_{a}, \sqrt{\lambda-\epsilon_{\beta}} \theta_{a}\right) \psi_{\beta}\left(x^{a}\right),\right. \\
& \left.t_{a, 1}\left(x_{a}, \sqrt{\lambda-\epsilon_{\alpha}} \omega_{a}\right) \psi_{\alpha}\left(x^{a}\right)\right) \\
l_{3}^{2}\left(\lambda, x_{a}, \omega_{a}, \theta_{a}\right)= & \chi\left(\cos \left(x_{a}, \omega_{a}\right)\right) e^{r\left(\lambda, x_{a}, \omega_{a}, \theta_{a}\right)} \\
& \times\left(\left(w_{a}(x)-1\right) m_{a, 2}\left(x_{a}, \sqrt{\lambda-\epsilon_{\beta}} \theta_{a}\right) \psi_{\beta}\left(x^{a}\right),\right. \\
& \left.t_{a, 1}\left(x_{a}, \sqrt{\lambda-\epsilon_{\alpha}} \omega_{a}\right) \psi_{\alpha}\left(x^{a}\right)\right) \\
l_{3}^{3}\left(\lambda, x_{a}, \omega_{a}, \theta_{a}\right)= & \chi\left(\cos \left(x_{a}, \omega_{a}\right)\right) e^{r\left(\lambda, x_{a}, \omega_{a}, \theta_{a}\right)} \times\left(m_{a, 2}\left(x_{a}, \sqrt{\lambda-\epsilon_{\beta}} \theta_{a}\right) \psi_{\beta}\left(x^{a}\right),\right. \\
& \left.\left(w_{a}(x)-1\right) t_{a, 1}\left(x_{a}, \sqrt{\lambda-\epsilon_{\alpha}} \omega_{a}\right) \psi_{\alpha}\left(x^{a}\right)\right) \\
l_{3}^{4}\left(\lambda, x_{a}, \omega_{a}, \theta_{a}\right)= & \chi\left(\cos \left(x_{a}, \omega_{a}\right)\right) e^{r\left(\lambda, x_{a}, \omega_{a}, \theta_{a}\right)} \\
& \times\left(\left(w_{a}(x)-1\right) m_{a, 2}\left(x_{a}, \sqrt{\lambda-\epsilon_{\beta}} \theta_{a}\right) \psi_{\beta}\left(x^{a}\right),\right. \\
& \left.\left(w_{a}(x)-1\right) t_{a, 1}\left(x_{a}, \sqrt{\lambda-\epsilon_{\alpha}} \omega_{a}\right) \psi_{\alpha}\left(x^{a}\right)\right)
\end{aligned}
$$

Remarquons que $\left(w_{a}(x)-1\right)$ étant supporté dans la région $\left\langle x^{a}\right\rangle \geq\left\langle x_{a}\right\rangle$ la fonction :

$$
e^{\epsilon_{0}\left\langle x_{a}\right\rangle}\left(w_{a}(x)-1\right) e^{-\epsilon_{0}\left\langle x^{a}\right\rangle}
$$

est bornée. Ainsi au vu du théorème 2.2 les termes $l_{3}^{2}, l_{3}^{3}$ et $l_{3}^{4}$ sont exponentiellement décroissants en $x_{a}$. Les intégrales correspondantes $L_{3}^{k}$, $(k=2,3,4)$ sont donc absolument convergentes définissant des fonctions $G^{s}\left(S_{a} \times S_{a}\right)$ holomorphes en $\lambda$ sur un domaine $\mathcal{D}_{\lambda^{\prime}, \epsilon}$. Seul le terme :

$$
L_{3}^{1}=\int_{\left|x_{a}\right| \geq R} e^{i x_{a}\left(\sqrt{\lambda-\epsilon_{\alpha}} \omega_{a}-\sqrt{\lambda-\epsilon_{\beta}} \theta_{a}\right)} l_{3}^{1}\left(\lambda, x_{a}, \omega_{a}, \theta_{a}\right) d x_{a}
$$

dont l'intégrande est holomorphe en $\left|x_{a}\right|$ reste à étudier.

Distinguons ici deux cas, selon que les canaux de réaction avant et après diffusion sont identiques, ou non, cette distinction ne portant que sur les énergies $\epsilon_{\alpha}$ et $\epsilon_{\beta}$, puisqu'on a déjà supposé que $a=b$.

Premier cas : $\epsilon_{\alpha}=\epsilon_{\beta}$.

Nous écrivons pour $\lambda$ réel :

$$
L_{3}^{1}=\left(\lambda-\epsilon_{\alpha}\right)^{-\frac{n_{a}}{2}} \int_{\left|y_{a}\right| \geq R \sqrt{\lambda-\epsilon_{\alpha}}} e^{i\left(\omega_{a}-\theta_{a}\right) \cdot y_{a}} l_{3}^{1}\left(\lambda, y_{a}\left(\lambda-\epsilon_{\alpha}\right)^{-\frac{1}{2}}, \omega_{a}, \theta_{a}\right) d y_{a}
$$

Notons $u\left(\lambda, y_{a}, \omega_{a}, \theta_{a}\right)=l_{3}^{1}\left(\lambda, y_{a}\left(\lambda-\epsilon_{\alpha}\right)^{-\frac{1}{2}}, \omega_{a}, \theta_{a}\right)$.

La fonction $u$ se prolonge holomorphiquement en $\lambda$ sur un domaine $\mathcal{D}_{\lambda^{\prime}, \epsilon}$ et vérifie :

$$
\left|\partial_{\omega_{a}}^{\alpha} \partial_{\theta_{a}}^{\beta} \partial_{y_{a}}^{\gamma} u\left(\lambda, y_{a}, \omega_{a}, \theta_{a}\right)\right| \leq C^{1+|\alpha|+|\beta|+|\gamma|}\left\langle y_{a}\right\rangle^{-\gamma} e^{C\left\langle y_{a}\right)^{1-\rho}}(|\alpha| !)^{s}(|\beta| !)^{s}(|\gamma| !)^{s}
$$


Décomposons avec $R_{1}$ assez grand $L_{3}^{1}$ en deux termes :

$$
\begin{aligned}
L_{3}^{1} & =\left(\lambda-\epsilon_{\alpha}\right)^{-\frac{n_{a}}{2}} \int_{R \sqrt{\lambda-\epsilon_{\alpha}} \leq\left|y_{a}\right| \leq R_{1}} e^{i\left(\omega_{a}-\theta_{a}\right) \cdot y_{a}} u\left(\lambda, y_{a}, \omega_{a}, \theta_{a}\right) d y_{a} \\
& +\left(\lambda-\epsilon_{\alpha}\right)^{-\frac{n_{a}}{2}} \int_{\left|y_{a}\right| \geq R_{1}} e^{i\left(\omega_{a}-\theta_{a}\right) \cdot y_{a}} u\left(\lambda, y_{a}, \omega_{a}, \theta_{a}\right) d y_{a}
\end{aligned}
$$

Le premier se prolonge au vu de (3.8) en $\lambda \in \mathcal{D}_{\lambda^{\prime}, \epsilon}$ comme une fonction $G^{s}\left(S_{a} \times S_{b}\right)$.

Pour le deuxième, que nous noterons $L_{3}^{\prime}$, nous distinguerons les cas $\rho \geq 1$ et $\rho<1$.

Si $\rho \geq 1$ l'estimation (3.8) est de type symbole pseudo-différentiel, et $L_{3}^{\prime}$ définit donc le noyau d'un opérateur continu de $C^{\infty}\left(S_{a}\right)$ dans $C^{\infty}\left(S_{a}\right)$ et de $D^{\prime}\left(S_{a}\right)$ dans $D^{\prime}\left(S_{a}\right)$, holomorphe en $\lambda \in \mathcal{D}_{\lambda^{\prime}, \epsilon^{\prime}}$.

Si $\rho<1$ nous remarquons que $u\left(\lambda, y_{a}, \omega_{a}, \theta_{a}\right)$ est supporté dans $\cos \left(y_{a}, \omega_{a}\right) \in\left[\sigma_{1}^{-}-\delta, \sigma_{1}^{+}+\delta\right]$ et ainsi que sur le support de $u$ :

$$
\partial_{\omega_{a}}\left[y_{a} \cdot\left(\omega_{a}-\theta_{b}\right)\right]=\left(y_{a} \cdot \omega_{a}\right) \omega_{a}-y_{a} \neq 0
$$

A $\lambda$ fixé on se trouve exactement dans le cadre du lemme 1.2.1 et du théorème 1.2 .2 de [C-Z], qui affirme que $L_{3}^{1}$, qui est rappelons-le défini comme une intégrale oscillante, est le noyau d'un opérateur borné de $G^{s}\left(S_{a}\right)$ dans $G^{s}\left(S_{a}\right)$ et de $\left(G^{s}\left(S_{a}\right)\right)^{\prime}$ dans $\left(G^{s}\left(S_{a}\right)\right)^{\prime}$. Ainsi ces opérateurs apparaissent comme étant une limite faible d'opérateurs holomorphes en $\lambda$ (uniformément par rapport à $\lambda$ ) et sont donc holomorphes en $\lambda$ sur un domaine $\mathcal{D}_{\lambda^{\prime}, \epsilon}$

Deuxième cas : $\epsilon_{\alpha} \neq \epsilon_{\beta}$

On peut supposer par exemple que $\epsilon_{\alpha}<\epsilon_{\beta}$, les canaux de réactions $\alpha$ et $\beta$ jouant au départ un rôle tout à fait symétrique.

Pour $\lambda$ réel si l'on choisit $\sigma_{1}^{-}$et $\sigma_{1}^{+}$assez proches de 1 , on a sur le support de $l_{3}^{1}\left(\lambda, x_{a}, \omega_{a}, \theta_{a}\right)$ :

$$
x_{a} \cdot\left(\sqrt{\lambda-\epsilon_{\alpha}} \omega_{a}-\sqrt{\lambda-\epsilon_{\beta}} \theta_{a}\right) \geq \nu\left|x_{a}\right| \quad ; \forall \omega_{a}, \theta_{a} \in S_{a} ; \nu>0
$$

Ainsi $l_{3}^{1}\left(\lambda, x_{a}, \omega_{a}, \theta_{a}\right)$ étant analytique en $\left|x_{a}\right|$ dans la région $\left|x_{a}\right| \geq R$ on peut faire le changement de contour d'intégration $x_{a} \rightarrow\left(1+i \epsilon \chi\left(\left|x_{a}\right| \geq R\right)\right) x_{a}$. L'intégrale définissant $L_{3}^{1}$ étant définie comme une intégrale oscillante il n'est pas difficile en effet de vérifier que la contribution à l'infini est bien nulle. Avec ce changement de contour $L_{3}^{1}$ devient alors une intégrale absolument convergente et définit une fonction $G^{s}\left(S_{a} \times S_{a}\right)$ qui se prolonge holomorphiquement en $\lambda$ sur un domaine $\mathcal{D}_{\lambda^{\prime}, \epsilon}$.

- Cas où $a \neq b$ 
Dans ce cas les espaces $X_{a}$ et $X_{b}$ sont distincts et l'on ne peut plus faire successivement le produit scalaire sur $X^{a}$ puis sur $X_{a}$ dans l'équation (3.4). Cependant nous avons pris le soin de construire nos symboles de telle sorte que les opérateurs $e^{\epsilon_{0}\left\langle x^{b}\right\rangle} m_{b, 2}\left(x_{b}, \sqrt{\lambda-\epsilon_{\beta}} \theta_{b}\right) \psi_{\beta}\left(x^{b}\right)$, $e^{\epsilon_{0}\left\langle x^{a}\right\rangle} m_{a, 1}\left(x_{a}, \sqrt{\lambda-\epsilon_{\alpha}} \omega_{a}\right) \psi_{\alpha}\left(x^{a}\right), \quad e^{\epsilon_{0}\left\langle x^{a}\right\rangle} D_{x^{a}} m_{a, 1}\left(x_{a}, \sqrt{\lambda-\epsilon_{\alpha}} \omega_{a}\right) \psi_{\alpha}\left(x^{a}\right)$ et $e^{\epsilon_{0}\left\langle x^{a}\right\rangle} t_{a, 1}\left(x_{a}, \sqrt{\lambda-\epsilon_{\alpha}} \omega_{a}\right) \psi_{\alpha}\left(x^{a}\right)$ soient uniformément bornés par rapport à $\lambda, x, \omega_{a}, \theta_{b}$ (Cf Théorème 2.2).

Ecrivons :

$$
\begin{aligned}
M_{1}= & \left(e^{-\epsilon_{0}\left\langle x^{b}\right\rangle} e^{i \phi_{b, 2}\left(x_{b}, \sqrt{\lambda-\epsilon_{\beta}} \theta_{b}\right)} \chi_{b_{1}}\left(\frac{x_{b}}{\left|x_{b}\right|}\right) w_{b}(x) e^{\epsilon_{0}\left\langle x^{b}\right\rangle} m_{b, 2}\left(x_{b}, \sqrt{\lambda-\epsilon_{\beta}} \theta_{b}\right) \psi_{\beta}\left(x^{b}\right),\right. \\
& e^{-\epsilon_{0}\left\langle x^{a}\right\rangle} e^{i \phi_{a, 1}\left(x_{a}, \sqrt{\lambda-\epsilon_{\alpha}} \omega_{a}\right)}\left(w_{a}(x) e^{\epsilon_{0}\left\langle x^{a}\right\rangle} t_{a, 1}\left(x_{a}, \sqrt{\lambda-\epsilon_{\alpha}} \omega_{a}\right)\right. \\
& \left.\left.+e^{\epsilon_{0}\left\langle x^{a}\right\rangle}\left[D_{x}^{2}, w_{a}(x)\right] m_{a, 1}\left(x_{a}, \sqrt{\lambda-\epsilon_{\alpha}} \omega_{a}\right)\right) \psi_{\alpha}\left(x^{a}\right)\right)_{L^{2}(X)}
\end{aligned}
$$

et notons que comme $a$ et $b$ correspondent ici à deux compositions différentes il existe une constante $\epsilon_{1}>0$ telle que :

$$
2 \epsilon_{1}\langle x\rangle \chi_{b_{1}}\left(\frac{x_{b}}{\left|x_{b}\right|}\right) \leq \epsilon_{0}\left(\left\langle x^{a}\right\rangle+\left\langle x^{b}\right\rangle\right), \quad \forall x \in X .
$$

Il vient alors :

$$
\begin{aligned}
M_{1}= & \left(e^{-\epsilon_{1}\left\langle x_{b}\right)} e^{i \phi_{b, 2}\left(x_{b}, \sqrt{\lambda-\epsilon_{\beta}} \theta_{b}\right)} K_{b, 2}\left(\lambda, x_{b}, \theta_{b}\right),\right. \\
& \left.e^{-\epsilon_{1}\left(x_{a}\right\rangle} e^{i \phi_{a, 1}\left(x_{b}, \sqrt{\left.\lambda-\epsilon_{\alpha} \omega_{a}\right)}\right.} K_{a, 1}\left(\lambda, x_{a}, \omega_{a}\right)\right)_{L^{2}(X)}
\end{aligned}
$$

où les symboles $K_{b, 2}\left(\lambda, x_{b}, \theta_{b}\right)$ et $K_{a, 1}\left(\lambda, x_{a}, \omega_{a}\right)$, définis comme des éléments de $G^{s}\left(K_{b}, L^{2}\left(X^{b}\right)\right)$ et $G^{s}\left(S_{a}, L^{2}\left(X^{a}\right)\right)$, sont holomorphes, en $\lambda \in \mathcal{D}_{\lambda^{\prime}, \epsilon}$, bornés uniformément par rapport à $\left\langle x_{b}\right\rangle$ et $\left\langle x_{a}\right\rangle$.

Il suit alors des propriétés des fonctions de phase $\phi_{a, j}$ que $M_{1}$ définit une fonction $G^{s}\left(S_{a} \times K_{b}\right)$, holomorphe en $\lambda$ sur un domaine de la forme $\mathcal{D}_{\lambda^{\prime}, \epsilon}$ (avec $\epsilon<<\epsilon_{1}$ ).

\subsection{Prolongement de $M_{2}$}

$\mathrm{Au}$ regard de l'expression de $M_{2}\left(\epsilon^{\prime}\right)$ il apparait clairement que pour construire un prolongement méromorphe en $\lambda$ de $M_{2}=\lim _{\epsilon^{\prime} \rightarrow 0^{+}} M_{2}\left(\epsilon^{\prime}\right)$ la difficulté principale vient d'une part bien sur de ce que l'existence même de cette limite que n'a pas encore été démontrée, mais surtout du fait que, de toute façon, on ne saurait prolonger méromorphiquement le terme $R(\lambda+i 0)$ sur un domaine du type $\mathcal{D}_{\lambda^{\prime}, \epsilon}$ à cause de la coupure due au spectre essentiel de 
$H$. Pour surmonter ces difficultés nous utiliserons les opérateurs de boostdistorsions, qui sont décrits dans l'appendice A.. Considérons donc une fonction de troncature $\chi$ vérifiant la condition (A.1) et telle que :

$$
\chi(x)=0 \quad \forall|x|<2 R
$$

Avec cette fonction de troncature nous définissons, pour $\theta$ et $k$ réels, l'opérateur $\tilde{W}(\theta, k)$, dit opérateur de boost-distorsion, par la définition A.1. Nous commençons alors par affirmer que :

\section{Proposition 3.2 -}

(i) Les vecteurs $\tilde{W}(\bar{\theta}, \bar{k}) s_{b, 2}(x)$ et $\tilde{W}(\theta, k) s_{a, 1}(x)$, considérés respectivement comme des éléments de $C^{0}\left(X_{b}, G^{s}\left(K_{b}, L^{2}\left(X^{b}\right)\right)\right)$ et de $C^{0}\left(X_{a}, G^{s}\left(S_{a}, L^{2}\left(X^{a}\right)\right)\right)$ pour $\theta, k$ réels, et $\lambda$ dans un domaine de la forme $\mathcal{D}_{\lambda^{\prime}, \epsilon^{\prime}}$ se prolongent, holomorphiquement, respectivement en $(\bar{\theta}, \bar{k}, \lambda)$ et en $(\theta, k, \lambda)$, sur un domaine de la forme $\{|\theta|<\epsilon\} \times,\{k \in \mathbb{C}\} \times \mathcal{D}_{\lambda^{\prime}, \epsilon}$.

(ii) Pour $\operatorname{Im}(\theta)>0$ assez petit, et $k$ tel que :

$\sigma_{K_{b}} \sup _{\lambda \in \mathcal{D}_{\lambda^{\prime}, \epsilon}}\left(\operatorname{Im}\left((1+\theta)\left(\lambda-\epsilon_{\beta}\right)^{\frac{1}{2}}\right)\right)<\operatorname{Im}(k)<\sigma_{1}^{-} \inf _{\lambda \in \mathcal{D}_{\lambda^{\prime}, \epsilon}}\left(\operatorname{Im}\left((1+\theta)\left(\lambda-\epsilon_{\alpha}\right)^{\frac{1}{2}}\right)\right)$

$\sup _{\theta_{b} \in \tilde{S}_{b}}\left\|\partial_{\theta_{b}}^{\alpha} \tilde{W}(\bar{\theta}, \bar{k}) s_{b, 2}(x)\right\|_{L^{2}\left(X^{b}\right)}$ et $\sup _{\omega_{a} \in S_{a}^{n_{a}-1}}\left\|\partial_{\omega_{a}}^{\alpha} \tilde{W}(\theta, k) s_{a, 1}\right\|_{L^{2}\left(X^{a}\right)}$ sont exponentiellement décroissants, respectivement en $\left\langle x_{b}\right\rangle$ et en $\left\langle x_{a}\right\rangle$, pour tout multiindice $\alpha$.

Remarque : Si l'on choisit $\epsilon$ assez petit, on peut toujours trouver un $k$ tel que l'inégalité (3.12) soit vérifiée. En effet si $\beta$ est un canal de réaction à deux amas, alors conventionellement $\sigma_{K_{b}}<0$ et $k=0$ convient. Si $\beta$ est un canal de réaction à plus de 2 amas alors d'après l'hypothèse $(E .5 .2)$ $\epsilon_{\alpha} \leq \epsilon_{\beta}$, et par construction $\sigma_{1}^{-}>\sigma_{K}$ ce qui donne pour $\operatorname{Im}(\theta)>0$ et $\epsilon$ assez petit :

$$
\sigma_{K_{b}} \sup _{\lambda \in \mathcal{D}_{\lambda^{\prime}, \epsilon}}\left(\operatorname{Im}\left((1+\theta)\left(\lambda-\epsilon_{\beta}\right)^{\frac{1}{2}}\right)\right)<\sigma_{1}^{-} \inf _{\lambda \in \mathcal{D}_{\lambda^{\prime}, \epsilon}}\left(\operatorname{Im}\left((1+\theta)\left(\lambda-\epsilon_{\alpha}\right)^{\frac{1}{2}}\right)\right)
$$

Démonstration de la proposition 3.2 :

Rappelons tout d'abord l'expression des vecteurs $s_{a, i}(x)$ :

$$
\begin{aligned}
s_{a, i}(x)= & w_{a}(x) \chi_{a_{1}}\left(\frac{x_{a}}{\left|x_{a}\right|}\right) e^{i \phi_{a, i}\left(x_{a}, \sqrt{\lambda-\epsilon_{\alpha}} \omega_{a}\right)} t_{a, i}\left(x_{a}, \sqrt{\lambda-\epsilon_{\alpha}} \omega_{a}\right) \psi_{\alpha}\left(x^{a}\right) \\
& +\left[D_{x}^{2}, w_{a}(x) \chi_{a_{1}}\left(\frac{x_{a}}{\left|x_{a}\right|}\right)\right] e^{i \phi_{a, i}\left(x_{a}, \sqrt{\lambda-\epsilon_{\alpha}} \omega_{a}\right)} m_{a, i}\left(x_{a}, \sqrt{\lambda-\epsilon_{\alpha}} \omega_{a}\right) \psi_{\alpha}\left(x^{a}\right)
\end{aligned}
$$


Nous noterons encore ici :

$$
\begin{aligned}
s_{a, i}(x)= & w_{a}(x) \chi_{a_{1}}\left(\frac{x_{a}}{\left|x_{a}\right|}\right) z_{1}\left(\lambda, x_{a}, x^{a}\right)-2 i \nabla_{x}\left(w_{a}(x) \chi_{a_{1}}\left(\frac{x_{a}}{\left|x_{a}\right|}\right)\right) . z_{2}\left(\lambda, x_{a}, x^{a}\right) \\
& -\triangle_{x}\left(w_{a}(x) \chi_{a_{1}}\left(\frac{x_{a}}{\left|x_{a}\right|}\right)\right) z_{3}\left(\lambda, x_{a}, x^{a}\right)
\end{aligned}
$$

où

$$
\begin{aligned}
& z_{1}\left(\lambda, x_{a}, x^{a}\right)=e^{i \phi_{a, i}\left(x_{a}, \sqrt{\lambda-\epsilon_{\alpha}} \omega_{a}\right)} t_{a, i}\left(x_{a}, \sqrt{\lambda-\epsilon_{\alpha}} \omega_{a}\right) \psi_{\alpha}\left(x^{a}\right) \\
& z_{2}\left(\lambda, x_{a}, x^{a}\right)=D_{x} e^{i \phi_{a, i}\left(x_{a}, \sqrt{\lambda-\epsilon_{\alpha}} \omega_{a}\right)} m_{a, i}\left(x_{a}, \sqrt{\lambda-\epsilon_{\alpha}} \omega_{a}\right) \psi_{\alpha}\left(x^{a}\right) \\
& z_{3}\left(\lambda, x_{a}, x^{a}\right)=e^{i \phi_{a, i}\left(x_{a}, \sqrt{\lambda-\epsilon_{\alpha}} \omega_{a}\right)} m_{a, i}\left(x_{a}, \sqrt{\lambda-\epsilon_{\alpha}} \omega_{a}\right) \psi_{\alpha}\left(x^{a}\right)
\end{aligned}
$$

Commençons par montrer qu'à $\omega_{a}$ fixé on peut construire un prolongement holomorphe en $(\lambda, \theta, k)$ des vecteurs $\tilde{W}(\theta, k) s_{a, i}$.

Nous savons d'après le théorème 2.2 que les applications :

$$
(\lambda, \mu, \theta) \rightarrow z_{i}\left(\lambda,(1+\mu) x_{a},(1+\theta) x^{a}\right) \in L^{2}\left(X^{a}\right)
$$

sont holomorphes, pour tout $x_{a} \in X_{a} \cup\left\{x_{a} \mid\left\langle x_{a}\right\rangle>R\right\}$, sur un domaine de la forme $\mathcal{D}_{\lambda^{\prime}, \epsilon} \times\{|\mu|<\epsilon\} \times\{|\theta|<\epsilon\}$.

Il en suit aisément que les applications :

$$
\begin{cases}\mathcal{D}_{\lambda^{\prime}, \epsilon} \times\{|\theta|<\epsilon\} & \rightarrow L^{2}\left(X^{a}\right) \\ (\lambda, \theta) & \rightarrow y_{i}(\lambda, \theta)=z_{i}\left(\lambda,(1+\theta \chi(x)) x_{a},(1+\theta \chi(x)) x^{a}\right)\end{cases}
$$

sont holomorphes, pour tout $x_{a} \in X_{a} \cap\left\{x_{a} \mid\left\langle x_{a}\right\rangle>R\right\}$. Pour montrer alors que les vecteurs $\tilde{W}(\theta, k) s_{a, i}$ se prolongent holomorphiquement en $(\lambda, \theta, k)$ il nous suffit d'écrire :

$$
\begin{aligned}
\tilde{W}(\theta, k) s_{a, i}(x)= & e^{-i k\langle x\rangle} J_{\theta}(x)^{\frac{1}{2}} w_{a}(x) \chi_{a_{1}}\left(\frac{x_{a}}{\left|x_{a}\right|}\right) y_{1}(\lambda, \theta) \\
& -2 i e^{-i k\langle x\rangle} J_{\theta}(x)^{\frac{1}{2}} \nabla_{x}\left(w_{a}(x) \chi_{a_{1}}\left(\frac{x_{a}}{\left|x_{a}\right|}\right)\right) \cdot y_{2}(\lambda, \theta) \\
& -e^{-i k\langle x\rangle} J_{\theta}(x)^{\frac{1}{2}} \triangle_{x}\left(w_{a}(x) \chi_{a_{1}}\left(\frac{x_{a}}{\left|x_{a}\right|}\right)\right) y_{3}(\lambda, \theta)
\end{aligned}
$$

Trois cas se présentent :

1) $x \in \operatorname{supp} w_{a} \cap \operatorname{supp} \chi$. Dans ce cas $\left\langle x_{a}\right\rangle>R$ et d'après ce qui précède $\tilde{W}(\theta, k) s_{a, i}(x) \in L^{2}\left(X^{a}\right)$ est holomorphe en $(\lambda, \theta, k)$ sur un domaine de la forme $\mathcal{D}_{\lambda^{\prime}, \epsilon} \times\{|\theta|<\epsilon\} \times\{k \in \mathbb{C}\}$.

2) $x \notin \operatorname{supp} w_{a}$. Le terme $\tilde{W}(\theta, k) s_{a, i}(x)$ est alors nul et l'holomorphie en $(\lambda, \theta, k)$ est évidente.

3) $x_{a} \notin \operatorname{supp} \chi$. Dans ce cas $\tilde{W}(\theta, k) s_{a, i}(x)$ ne dépend pas de $\theta$ et l'holomorphie en $\theta$ est triviale. Celle en $\lambda$ et $k$ ne posent aucun problème, d'après le théorème 2.2 .

Pour vérifier que le prolongement ainsi construit définit bien une fonction 
holomorphe à valeurs dans l'espace de Gevrey considéré, nous constaterons sans difficulté à la vue des expressions donnant les termes $z_{i}$, qu'à $\lambda$ fixé le terme $\tilde{W}(\theta, k) s_{a, i}$ est Gevrey $s$ en $\omega_{a}$, et que de plus on a des estimations localement uniformes en $\lambda$ pour les semi-normes correspondantes.

Ceci achève la démonstration de $(i)$.

Il ne nous reste plus qu'à montrer les décroissances exponentielles annoncées dans $(i i)$.

D'après la construction des symboles et des fonctions de phase, nous avons dans les régions où sont supportés les symboles $m_{a, 1}$ et $m_{b, 2}$ les estimations uniformes :

$$
\left.\phi_{a}\left(x_{a}, \xi_{a}\right)=\left(x_{a} \cdot \xi_{a}+O\left(\left\langle x_{a}\right\rangle^{1-\rho}\right)\right)\right)
$$

et

$$
\phi_{b}\left(x_{b}, \xi_{b}\right)=\left(x_{b} \cdot \xi_{b}+O\left(\left\langle x_{a}\right\rangle^{1-\rho}\right)\right)
$$

Posons par la suite :

$$
\begin{aligned}
& C_{\theta, \epsilon}=\frac{1}{2}\left[\operatorname{Im}(k)-\sigma_{K_{b}} \sup _{\lambda \in \mathcal{D}_{\lambda^{\prime}, \epsilon}}\left(\operatorname{Im}\left((1+\theta)\left(\lambda-\epsilon_{\beta}\right)^{\frac{1}{2}}\right)\right)\right] \\
& C_{\theta, \epsilon}^{\prime}=\frac{1}{2}\left[\sigma_{1}^{-} \inf _{\lambda \in \mathcal{D}_{\lambda^{\prime}, \epsilon}}\left(\operatorname{Im}\left((1+\theta)\left(\lambda-\epsilon_{\alpha}\right)^{\frac{1}{2}}\right)\right)-\operatorname{Im}(k)\right] \\
& C_{\theta, \epsilon}^{\prime \prime}=\sup _{\lambda \in \mathcal{D}_{\lambda^{\prime}, \epsilon}}\left(\operatorname{Im}\left((1+\theta)\left(\lambda-\epsilon_{\alpha}\right)^{\frac{1}{2}}\right)\right)+|\operatorname{Im}(k)|
\end{aligned}
$$

D'après (3.12) ces constantes sont strictement positives, et :

$$
\lim _{\epsilon, \operatorname{Im}(\theta) \rightarrow 0} C_{\theta, \epsilon}^{\prime \prime}=0
$$

En utilisant (3.13) et (3.14) il vient :

$$
e^{-i k\langle x\rangle} U_{\theta} e^{i \phi_{a}\left(x_{a}, \sqrt{\lambda-\epsilon_{\alpha}} \omega_{a}\right)}=\left\{\begin{array}{lll}
O\left(e^{C_{\theta, \epsilon}^{\prime \prime}\langle x\rangle}\right) & \text { pour } & \cos \left(x_{a}, \omega_{a}\right) \in\left[-1, \sigma_{1}^{-}\right] \\
O\left(e^{-C_{\theta, \epsilon}^{\prime}\left\langle x_{a}\right\rangle}\right) & \text { pour } & \cos \left(x_{a}, \omega_{a}\right) \in\left[\sigma_{1}^{-}, 1\right]
\end{array}\right.
$$

et

$$
e^{-i \bar{k}\langle x\rangle} \tilde{U}_{\bar{\theta}} e^{i \phi_{b}\left(x_{b}, \sqrt{\lambda-\epsilon_{\beta}} \theta_{b}\right)}=\left\{\begin{array}{lll}
O\left(e^{-C_{\theta, \epsilon}\left\langle x_{b}\right\rangle}\right) & \text { pour } & \cos \left(x_{b}, \theta_{b}\right) \in\left[-1, \sigma_{K_{b}}\right] \\
O\left(e^{C_{\theta, \epsilon}^{\prime \prime}\langle x\rangle}\right) & \text { pour } \cos \left(x_{b}, \theta_{b}\right) \in\left[\sigma_{K_{b}}, 1\right]
\end{array}\right.
$$

D'autre part nous avons les estimations uniformes en $\lambda$ et $\theta$ : 


$$
\begin{aligned}
& \left\|e^{\epsilon_{o}\left\langle x^{a}\right\rangle} U_{\theta}\left(t_{a, 1}\left(x_{a}, \sqrt{\lambda-\epsilon_{\alpha}} \omega_{a}\right) \psi_{\alpha}\left(x^{a}\right)\right)\right\|_{L^{2}\left(X^{a}\right)}= \\
& \left\{\begin{array}{lc}
O(1) & \text { pour } \cos \left(x_{a}, \omega_{a}\right) \in\left[\sigma_{1}^{-}, \sigma_{1}^{+}\right] \\
O\left(e^{-\epsilon_{0}\left\langle x_{a}\right\rangle}\right) & \text { sinon }
\end{array}\right.
\end{aligned}
$$

Comme les dérivées de $w_{a}$ sont supportées dans une région où $\left\langle x_{a}\right\rangle \simeq\left\langle x^{a}\right\rangle$ nous avons aussi :

$$
\left\|e^{\epsilon_{0}\left\langle x^{a}\right\rangle} U_{\theta}\left[D_{x}^{2}, w_{a}(x)\right]\left(m_{a, 2}\left(x_{a}, \sqrt{\lambda-\epsilon_{\alpha}} \omega_{a}\right) \psi_{\alpha}\left(x^{a}\right)\right)\right\|_{L^{2}\left(X^{a}\right)}=O\left(e^{-\epsilon_{0}\left\langle x_{a}\right\rangle}\right)
$$

Remarquons que $\alpha$ étant par hypothèse un canal de réaction à deux amas $\chi_{a_{1}}=1$. Des estimations (3.15), (3.17) et (3.18) nous en déduisons que :

$$
\left\|\tilde{W}(\theta, k) s_{a, 1}(x)\right\|_{L^{2}\left(X^{a}\right)}=O\left(e^{-\epsilon\left\langle x_{a}\right\rangle}\right)
$$

Pour le canal de sortie nous avons :

$$
\begin{aligned}
\left\|e^{\epsilon_{0}\left\langle x^{b}\right\rangle} U_{\theta}\left(t_{b, 2}\left(x_{b}, \sqrt{\lambda-\epsilon_{\beta}} \theta_{b}\right) \psi_{\beta}\left(x^{b}\right)\right)\right\|_{L^{2}\left(X^{a}\right)}= \\
\left\{\begin{array}{lc}
O(1) & \text { pour } \cos \left(x_{b}, \theta_{b}\right) \in\left[\sigma_{2}^{-}, \sigma_{2}^{+}\right] \\
O\left(e^{-\epsilon_{0}\langle x\rangle}\right) & \text { sinon }
\end{array}\right.
\end{aligned}
$$

Utilisons maintenant les propriétés de support des dérivées de $w_{b}(x)$ et $\chi_{b_{1}}\left(\frac{x_{b}}{\left|x_{b}\right|}\right)$, pour estimer le terme restant. Celles de $w_{b}(x)$ sont supportées là où $\left\langle x_{b}\right\rangle \simeq\left\langle x^{b}\right\rangle$, et celles de $\chi_{b_{1}}\left(\frac{x_{b}}{\left|x_{b}\right|}\right)$ dans la région $\left|\cos \left(x_{b}, \theta_{b}\right)\right| \leq \sigma_{K_{b}}$, pour tout $\theta_{b} \in K_{b}$. Nous obtenons donc:

$$
\begin{array}{r}
U_{\bar{\theta}}\left(\left[D_{x_{b}}^{2}, w_{b} \chi_{b_{1}}\left(\frac{x_{b}}{\left|x_{b}\right|}\right)\right] m_{b, 1}\left(x_{b}, \sqrt{\lambda-\epsilon_{\beta}} \theta_{b}\right) \psi_{\beta}\left(x^{b}\right)\right) \|_{L^{2}\left(X^{b}\right)}= \\
\left\{\begin{array}{lcc}
O(1) & \text { pour } & \left|\cos \left(x_{b}, \theta_{b}\right)\right| \leq \sigma_{K_{b}} \\
O\left(e^{-\epsilon_{0}\langle x\rangle}\right) & \text { sinon }
\end{array}\right.
\end{array}
$$

et ainsi, en utilisant (3.16) :

$$
\left\|\tilde{W}(\bar{\theta}, \bar{k}) s_{b, 2}(x)\right\|_{L^{2}\left(X^{b}\right)}=O\left(e^{-\epsilon\left\langle x_{b}\right\rangle}\right)
$$

Les estimations (3.19) et (3.22) étant uniformes en $\omega_{a} \in S^{a}$ et $\theta_{b} \in K_{b}$ elles donnent le résultat de la proposition 3.2 pour $|\alpha|=0$. Les autres cas se traitent de même.

Nous avons maintenant, avec la proposition 3.2 et l'appendice A., tous les éléments nécessaires à la construction d'un prolongement méromorphe du terme $M_{2}$. 
Pour $\lambda \in[d, M]$ reprenons l'expression :

$$
M_{2}\left(\lambda, \epsilon^{\prime}\right):=M_{2}\left(\epsilon^{\prime}\right)=\left(s_{b, 2}(x), R\left(\lambda+i \epsilon^{\prime}\right), s_{a, 1}(x)\right)_{L^{2}(X)}
$$

dont on va montrer que la limite pour $\epsilon^{\prime} \rightarrow 0$ est bien définie et qu'elle se prolonge méromorphiquement en $\lambda$ sur un voisinage de la forme $\mathcal{D}_{\lambda^{\prime}, \epsilon}$, pour tout $\lambda^{\prime} \in[d, M]$.

Choisissons $\gamma \in] \sigma_{K_{b}} \sqrt{\lambda^{\prime}-\epsilon_{\beta}}, \sigma_{1}^{-} \sqrt{\lambda^{\prime}-\epsilon_{\alpha}}\left[\cap \mathbb{R}^{+}\right.$. (On pourra prendre en particulier $\gamma=0$ lorsque $\beta$ est un canal de réaction à deux amas.)

Pour $\theta$ réel assez petit, $\tilde{W}(\theta, \gamma \theta)$ est un opérateur unitaire et nous avons encore :

$$
M_{2}\left(\lambda, \epsilon^{\prime}\right)=\left(\tilde{W}(\bar{\theta}, \gamma \bar{\theta}) s_{b, 2}(x), \tilde{R}_{W}\left(\lambda+i \epsilon^{\prime}\right), \tilde{W}(\theta, \gamma \theta) s_{a, 1}(x)\right)_{L^{2}(X)}
$$

où l'on a noté $\tilde{R}_{W}\left(\lambda^{\prime}+i \epsilon^{\prime}\right)=\left(\tilde{H}_{W}-\lambda^{\prime}-i \epsilon^{\prime}\right)^{-1}$ et $\tilde{H}_{W}=\tilde{W}(\theta, \gamma \theta) H(\tilde{W}(\theta, \gamma \theta))^{-1}$.

Nous savons par le théorème (A.3) que l'opérateur $\tilde{H}_{W}$ se prolonge holomorphiquement sur un domaine $\{|\theta|<\epsilon\}$ et que pour $\epsilon$ assez petit :

$$
\lambda+i \epsilon^{\prime} \notin \sigma_{e}\left(\tilde{H}_{W}\right) \quad, \forall|\theta|<\epsilon, \quad \operatorname{Im}(\theta)>0
$$

D'autre part, avec le choix $k=\gamma \theta$ la condition (3.12) est toujours vérifiée si l'on se limite aux $\lambda$ réels.

Ainsi la fonction :

$$
f\left(\lambda, \theta, \epsilon^{\prime}\right):=\left(\tilde{W}(\bar{\theta}, \gamma \bar{\theta}) s_{b, 2}(x), \tilde{R}_{W}\left(\lambda+i \epsilon^{\prime}\right), \tilde{W}(\theta, \gamma \theta) s_{a, 1}(x)\right)_{L^{2}(X)}
$$

est holomorphe en $\theta$ sur un domaine $\{|\theta|<\epsilon, \operatorname{Im}(\theta)>0\}$ pour tout $\lambda$ réel proche de $\lambda^{\prime}$. De plus (3.24) nous donne :

$$
\lim _{\operatorname{Im}(\theta) \rightarrow 0} f\left(\lambda, \theta, \epsilon^{\prime}\right)=M_{2}\left(\lambda, \epsilon^{\prime}\right)
$$

et cette limite est donc indépendante de $\operatorname{Re}(\theta)$. A $\lambda$ et $\epsilon^{\prime}$ fixés, quitte à multiplier $f\left(\lambda, \theta, \epsilon^{\prime}\right)$ par une constante, nous pouvons supposer que cette limite est réelle et construire un prolongement holomorphe en $\theta$ de $f\left(\theta, \lambda, \epsilon^{\prime}\right)$ sur le disque $\{|\theta|<\epsilon\}$ en posant $f\left(\bar{\theta}, \lambda, \epsilon^{\prime}\right)=\overline{f\left(\theta, \lambda, \epsilon^{\prime}\right)}$. Etant constant sur l'axe réel ce prolongement n'est rien d'autre qu'une fonction constante. Nous montrons ainsi que notre fonction d'origine, $f\left(\theta, \lambda, \epsilon^{\prime}\right)$, est indépendante de $\theta$ et égale à $M_{2}\left(\lambda, \epsilon^{\prime}\right)$.

L'expression (3.24) reste donc valable sur le demi-disque $\{|\theta|<\epsilon, \operatorname{Im}(\theta)>0\}$, pour tout $\lambda$ réel suffisamment proche de $\lambda^{\prime}$.

Fixons maintenant $\theta$ dans ce demi-disque. Il existe un domaine $\mathcal{D}_{\lambda^{\prime}, \epsilon}$ et une constante $\epsilon^{\prime \prime}$ sur lequel la condition (3.12) est vérifiée, et tel que 
$\mathcal{D}_{\lambda^{\prime}, \epsilon} \cap\left(\sigma_{e}\left(\tilde{H}_{W}\right)-i \epsilon^{\prime}\right)=\emptyset$ pour tout $\left|\epsilon^{\prime}\right|<\epsilon^{\prime \prime}$. D'après le théorème A.3, le terme $\tilde{R}_{W}\left(\lambda+i \epsilon^{\prime}\right)$ se prolonge méromorphiquement en $\lambda+i \epsilon^{\prime}$ comme un opérateur borné, et la limite $\lim _{\epsilon^{\prime} \rightarrow 0^{+}} \tilde{R}_{W}\left(\lambda+i \epsilon^{\prime}\right)$ définit donc une fonction méromorphe dont les pôles sont des valeurs propres de $\tilde{H}_{\theta}$. (En particulier si $\lambda$ est réel cette limite est bien définie et l'on voit que l'existence supposée de la limite de $M_{2}\left(\epsilon^{\prime}\right)$ ne pose effectivement aucune difficulté.) Utilisant maintenant la proposition 3.2 il apparait clairement que la fonction $f\left(\theta, \lambda, 0^{+}\right)$, donc $M_{2}$, se prolonge méromorphiquement en $\lambda \in \mathcal{D}_{\lambda^{\prime}, \epsilon}$ comme une fonction à valeur dans $G^{s}\left(S_{a} \times K_{b}\right)$, fonction dont les pôles sont nécessairement des valeurs propres de $\tilde{H}_{\theta}$, c'est à dire des résonances du Hamiltonien. 



\section{Démonstration du théorème $\mathbf{1 . 1 0}$}

L'idée de la démonstration suit la remarque suivante : dans le développement précédent la régularité des termes $M_{1}$ et $M_{2}$ ne semble être conditionnée que par la régularité en $\omega_{a}, \theta_{b}$ des fonctions de troncature utilisées dans la construction des opérateurs d'onde. Or les opérateurs d'onde, et par conséquent les opérateurs de diffusion $S_{\alpha \beta}$, ne sont pas modifiés par le choix de ces fonctions de troncature (à condition bien sûr de respecter les conditions de support). On pourra donc utiliser cette invariance pour montrer l'analyticité des noyaux de $S_{\alpha \beta}(\lambda)$ et de ses résidus. En particulier on s'attend assez naturellement à ce qu'en prenant des fonctions de troncature dans différentes classes on puisse en déduire que ces noyaux soient dans des classes de fonctions dont l'intersection est incluse dans l'ensemble des fonctions analytiques, et montrer ainsi qu'ils sont analytiques.

Pour mettre cela en forme nous posons quelques définitions supplémentaires:

Définition 4.1 - On note $\mathcal{G}$ l'ensemble des suites $\left(w_{k}\right)$ de $\mathbb{R}_{+}^{*}$ logarithmiquement convexes telles que:

$$
\sum_{k=0}^{+\infty} \frac{w_{k}}{w_{k+1}}<+\infty
$$

Définition 4.2 - Pour $w \in \mathcal{G}$ et $\Omega$ un ouvert de $\mathbb{R}^{n}$ on note $\mathcal{C}_{w}(\Omega)$ la classe de fonctions non quasi-analytiques définie par:

$$
\mathcal{C}_{w}(\Omega)=\left\{f \in C^{\infty}(\Omega) \mid \exists C \text { telle que }\left|f^{(k)}(x)\right| \leq C^{k+1} w_{k} \quad \forall x \in \Omega\right\}
$$

On a alors les propriétés suivantes : 
Proposition 4.3 - (i) Pour tout ouvert $\Omega \subset \mathbb{R}^{n}$, tout $s>1$

$$
\cap_{w \in \mathcal{G}}\left(\mathcal{C}_{w} \cap G^{s}\right)(\Omega) \subset a(\Omega)
$$

l'ensemble des fonctions analytiques sur $\Omega$.

(ii) $\forall w \in \mathcal{G}$ il existe des fonctions de troncature sur $S$ dans $\left(\mathcal{C}_{w} \cap G^{s}\right)(S)$

Démonstration : Commençons par prouver (i), qui n'est qu'une conséquence directe du théorème de $\mathrm{Bang}[\mathrm{Bg}]$. Ce dernier affirme en effet que :

$$
\cap_{w \in \mathcal{G}} \mathcal{C}_{w}(\Omega) \subset a(\Omega)
$$

et il nous suffit donc de montrer que :

$$
\forall w \in \mathcal{G}, s>1, \exists t \in \mathcal{G} \text { tel que } \mathcal{C}_{t}(\Omega) \subset\left(\mathcal{C}_{w} \cap G^{s}\right)(\Omega)
$$

Cela revient donc à montrer que pour toute suite $w \in \mathcal{G}$ et tout réel $s>1$ on peut construire une suite $t \in \mathcal{G}$ telle que $t_{k} \leq \inf \left(w_{k},(k !)^{s}\right)$.

Pour cela définissons des fonctions $u_{1}$ et $u_{2}$, continues, affines sur les intervalles $[k, k+1[$ et telles que :

$$
u_{1}(k)=\log w_{k} \quad \text { et } \quad u_{2}(k)=s \log (k !) \quad \forall k \in \mathbb{N}
$$

$u_{1}$ et $u_{2}$ sont des fonctions convexes car $\left(w_{k}\right)$ et $(k !)^{s}$ sont des éléments de $\mathcal{G}$. D'autre part :

$$
\int_{0}^{+\infty} e^{-u_{1}^{\prime}(x)} d x=\sum_{k=0}^{+\infty} \int_{k}^{k+1} e^{-u_{1}^{\prime}(x)} d x=\sum_{k=0}^{+\infty} \frac{w_{k}}{w_{k+1}}<\infty
$$

le même type d'estimation tenant aussi pour $u_{2}$.

Appelons alors $v$ l'enveloppe convexe de $u_{1}$ et $u_{2}$. La fonction $v$ est une fonction convexe et telle que :

$$
v(x) \leq \inf \left(u_{1}(x), u_{2}(x)\right) \text { et } v^{\prime}(x) \geq \inf \left(u_{1}^{\prime}(x), u_{2}^{\prime}(x)\right)
$$

Ainsi $\int_{0}^{+\infty} e^{-v^{\prime}(x)} d x<\infty$ et la suite $t$ définie par $t_{k}=e^{v(k)}$ répond au problème posé.

Le point (ii) découle immédiatement de (4.2), et du fait qu'il existe des fonctions de troncature dans toutes les classes $\mathcal{C}_{t}(\Omega)$.

\subsection{Démonstration de (2)}

Soit $\lambda_{0}$ un pôle de $S_{\alpha, \beta}(\lambda)$ et $Q_{i_{0}}$ les résidus correspondants. Pour montrer que les opérateurs $Q_{i_{0}}$ ont des noyaux analytiques sur $\left(S_{a} \times \stackrel{\circ}{K_{b}}\right)$ il nous suffit, 
d'après la proposition 4.3 de montrer que ces noyaux sont dans les classes $\left(\mathcal{C}_{w} \cap G^{s}\right)\left(S_{a} \times \stackrel{\circ}{K}_{b}\right)$, pour un réel $s>1$ et pour tout $w \in \mathcal{G}$.

Choisissons $\theta$ et $k$ complexes tels que la condition (3.12) soit satisfaite. Prenons donc $w \in \mathcal{G}$ et $s \in] 1,(1-\rho)^{-1}$ [, quelconques, et choisissons les fonctions de troncature intervenant dans les équations qui définissent les $\phi_{a, j}$ et $m_{a, j}$ dans la classe $\left(\mathcal{C}_{w} \cap G^{s}\right)$. Au vu de ces équations et des propriétés d'analyticité des autres termes, il est clair que l'on peut écrire :

$$
\tilde{W}(\theta, k) s_{a, 2}=\left(\chi_{1}\left(\omega_{a}, \frac{x_{a}}{\left|x_{a}\right|}\right) p_{1}\left(\lambda, x_{a}, \omega_{a}\right)+\chi_{2}\left(\omega_{a}, \frac{x_{a}}{\left|x_{a}\right|}\right) p_{2}\left(\lambda, x_{a}, \omega_{a}\right)\right) \chi_{a_{0}}\left(\omega_{a}\right)
$$

où les fonctions de troncature $\chi_{1}$ et $\chi_{2}$, choisies ici dans $\left(\mathcal{C}_{w} \cap G^{S}\right)\left(S_{a}\right)$, sont de supports inclus respectivement dans les régions $\left.\cos \left(\omega_{a}, x_{a}\right) \in\right] \sigma_{j}^{-}, \sigma_{j}^{-}+\delta[$ et $\left.\cos \left(\omega_{a}, x_{a}\right) \in\right] \sigma_{j}^{+}-\delta, \sigma_{j}^{+}\left[\right.$, et où les fonctions $p_{1}$ et $p_{2}$, à valeurs dans $L^{2}\left(X^{a}\right)$ sont analytiques en $\omega_{a}$. Ainsi au vu de la proposition 3.2 il vient en utilisant les estimations de Cauchy :

$$
\left\|\partial_{\omega_{a}}^{\alpha} p_{i}\left(\lambda, x_{a}, \omega_{a}\right)\right\|_{L^{2}\left(X^{a}\right)} \leq C^{\alpha+1} \alpha ! e^{-\epsilon_{2}\left\langle x_{a}\right\rangle}
$$

sur le support de $\chi_{i}$.

En remarquant que $w \in \mathcal{G} \Rightarrow \frac{w_{k}}{w_{k+i}} \leq C^{i} \frac{k !}{(k+i) !}$ pour une constante $C$ assez grande il vient :

$$
\left\|\partial_{\omega_{a}}^{\alpha} \tilde{W}(\theta, k) s_{a, 2}\right\|_{L^{2}(X)} \leq C^{\alpha+1} w_{|\alpha|}
$$

$\tilde{W}(\theta, k) s_{a, 2}$ est donc dans la classe $\mathcal{C}_{w}\left(S_{a}\right)$. De même on montre que $\tilde{W}(\bar{\theta}, \bar{k}) s_{b, 1}$ est dans la classe $\mathcal{C}_{w}\left(\stackrel{\circ}{K}_{b}\right)$ et par la même que $M_{2} \in \mathcal{C}_{w}\left(S_{a} \times \stackrel{\circ}{K}_{b}\right)$. On a déjà vu d'autre part qu'avec ce choix de troncature que $M_{2} \in G^{s}\left(S_{a} \times \stackrel{\circ}{K_{b}}\right)$.

Le terme $M_{1}$ étant holomorphe en $\lambda$ il est clair que les résidus de $S_{\alpha, \beta}(\lambda)$ sont identiques aux résidus de l'opérateur de noyau $M_{2}$ et ont donc des noyaux dans $\left(\mathcal{C}_{w} \cap G^{s}\right)\left(S_{a} \times \stackrel{\circ}{K}_{b}\right)$.

\subsection{Démonstration de (1)}

Nous procédons ici de façon tout à fait similaire en montrant que le noyau de $S_{\alpha, \beta}(\lambda)$ est dans toute les classes $\left(\mathcal{C}_{w} \cap G^{s}\right)(\Omega)$ où $\Omega=S_{a} \times \stackrel{\circ}{K}_{b}$ si $\alpha \neq \beta$ et $\Omega=S_{a} \times S_{b}\left\{\omega_{a}=\theta_{b}\right\}$ si $\alpha=\beta$.

Prenons donc un réel $s \in] 1,(1-\rho)^{-1}$ [ et une suite $w$ de $\mathcal{G}$ fixés, et choisissons les fonctions de troncature dans la classe $\mathcal{C}_{w} \cap G^{s}$. Nous venons de voir qu'avec ce choix de troncatures $M_{2} \in\left(\mathcal{C}_{w} \cap G^{s}\right)\left(S_{a} \times \stackrel{\circ}{K_{b}}\right)$. Seul le terme $M_{1}$ reste donc à étudier. Nous distinguons là aussi les cas $a=b$ et $a \neq b$. 
- Cas $a=b$

Si l'on reprend la démarche suivie dans le paragraphe "prolongement de $M_{1}$ " on constate aisément qu'avec ce choix de troncature les termes $L_{0}, L_{1} L_{2}$, et $L_{3}$ si $\epsilon_{\alpha} \neq \epsilon_{\beta}$, qui sont définis par des intégrales absolument convergentes, sont dans $\left(\mathcal{C}_{w} \cap G^{s}\right)\left(S_{a} \times S_{a}\right)$.

Seul le terme $L_{3}$ dans le cas où $\epsilon_{\alpha}=\epsilon_{\beta}$ mérite un peu plus d'attention. Prenons donc $\left(\omega_{a}^{0}, \theta_{a}{ }^{0}\right)$ un point de $\left(S_{a} \times S_{a}\right)$ donné et remarquons que pour $\lambda$ réel, si $\alpha \neq \beta$ ou si $\omega_{a}{ }^{0} \neq \theta_{a}{ }^{0}$, on peut toujours choisir $\sigma_{2}^{ \pm}$et $\delta$ tels qu'il existe un voisinage $\Omega$ de $\left(\omega_{a}{ }^{0}, \theta_{a}{ }^{0}\right), \tau= \pm 1$ et $\nu>0$ tels que :

$$
\left.\begin{array}{c}
\left(\omega_{a}, \theta_{a}\right) \in O \\
\text { et } \\
x_{a} \in \operatorname{supp}\left(l_{3}\left(\lambda, x_{a}, \omega_{a}, \theta_{a}\right)\right)
\end{array}\right\} \Rightarrow \tau x_{a} \cdot\left(\sqrt{\lambda-\epsilon_{\alpha}} \omega_{a}-\sqrt{\lambda-\epsilon_{\beta}} \theta_{a}\right) \geq \nu\left|x_{a}\right|
$$

Ainsi $l_{3}\left(\lambda, x_{a}, \omega_{a}, \theta_{a}\right)$ étant analytique en $\left|x_{a}\right|$ dans la région $\left|x_{a}\right| \geq R$ on peut faire le changement de contour d'intégration $y \rightarrow(1+i \tau \epsilon \chi(|y| \geq R)$. L'intégrale définissant $L_{3}$ devient alors absolument convergente, et donne donc un terme de $\left(\mathcal{C}_{w} \cap G^{s}\right)(\Omega)$.

- Cas $a \neq b$

Dans ce cas l'expression (3.10) fait apparaître une intégrale absolument convergente, les termes $K_{b, 1}\left(\lambda, x_{b}, \theta_{b}\right)$ et $K_{a, 2}\left(\lambda, x_{a}, \omega_{a}\right)$ étant pour leur part, avec ce choix de troncature dans $\mathcal{C}_{w} \cap G^{s}\left(\stackrel{\circ}{K}_{b}\right)$ et $\mathcal{C}_{w} \cap G^{s}\left(S_{a}\right)$. Le terme $M_{1}$ est donc évidemment dans la classe $\left(\mathcal{C}_{w} \cap G^{s}\right)\left(S_{a} \times \stackrel{\circ}{K}_{b}\right)$, ce qui achève la démonstration. 


\section{A. Appendice : Les "boost-distorsions" et le problème à $\mathbf{N}$ corps.}

L'outil que nous élaborons est en quelque sorte un prolongement, ou plutôt une combinaison, des développements de Hunziker [Hu] et de Combes et Thomas [Co-T], qui traitent respectivement des distorsions analytiques et des "boost-dilatations" pour les problèmes à N corps. Notre développement ici est nouveau étant donné que l'on considère des "boosters" légèrement différents de ceux de Combes et Thomas, et surtout qu'il place dans le même cadre distorsions et "boost-dilatations".

Posons quelques définitions.

Définition A.1 - Pour $\theta, k \in \mathbb{R}$, et toute fonction de troncature $\chi \in C^{\infty}\left(X, \mathbb{R}^{+}\right)$vérifiant :

$$
\begin{cases}|\chi(x)-\chi(y)| \leq|x-y| & \forall x, y \in X \\ \chi(x)=1 & \forall|x|>4 R\end{cases}
$$

nous définissons :

1) L'opérateur de distorsion $\tilde{U}_{\theta}$ par :

$$
\tilde{U}_{\theta}\left\{\begin{array}{l}
L^{2}(X) \rightarrow L^{2}(X) \\
\psi(x) \rightarrow J_{\theta}(x)^{\frac{1}{2}} \psi(x+\theta x \chi(x))
\end{array}\right.
$$

où $J_{\theta}(x)$ est le Jacobien de l'application $x \rightarrow x+\theta x \chi(x)$.

2) L'opérateur de boost-distorsion $\tilde{W}(\theta, k)$ par :

$$
\tilde{W}(\theta, k)\left\{\begin{array}{l}
L^{2}(X) \rightarrow L^{2}(X) \\
\psi(x) \rightarrow e^{-i k\langle x\rangle}\left(\tilde{U}_{\theta} \psi\right)(x)
\end{array}\right.
$$


Pour $|\theta|$ assez petit $\tilde{U}_{\theta}$ et $\tilde{W}(\theta, k)$ sont des opérateurs unitaires.

Définition A.2 - Nous notons $F$ l'ensemble des fonctions entières de $\mathbb{C}^{n}$ qui sont des $O\left(e^{-\alpha\langle z\rangle^{2}}\right), \alpha>0$, dans un cône de la forme $\left\{z \in \mathbb{C}^{n}|| \operatorname{Im} z \mid \leq \epsilon\langle\operatorname{Re}(z)\rangle\right\}$ et nous définissons l'ensemble A par :

$$
A:=\left\{\phi \in L^{2}(X) \mid \phi(x)=f(x) \text { pour une fonction } f \in F\right\}
$$

Nous énonçons alors le théorème suivant :

Théorème A.3 - Sous les hypothèses $(H)$, pour toute fonction $\chi$ vérifiant les conditions (A.1):

(i) La famille d'opérateurs $\tilde{H}_{W}=\tilde{W}(\theta, k) H \tilde{W}(\theta, k)^{-1}$ se prolonge holomorphiquement pour $\theta$ et $k$ complexes, $|\theta|$ assez petit, et définit alors une famille holomorphe de type $A$, de domaine $H^{0,2}$.

(ii) $\sigma_{d}\left(\tilde{H}_{W}\right)$ est indépendant de la fonction $\chi$.

(iii) $\sigma_{e}\left(\tilde{H}_{W}\right)$ le spectre essentiel de $\tilde{H}_{W}$ est inclus dans :

$$
S=\cup_{a}\left\{\sigma_{d}\left(H_{\theta}^{a}\right)+\frac{1}{(1+\theta)^{2}} P(k)\right\}
$$

où $P(k)$ est le paraboloïde d'équation :

$$
(\operatorname{Im}(z))^{2} \leq 4 c^{2}\left(\operatorname{Re}(z)+c^{2}\right) \quad ; \quad c=(\operatorname{Im}(k))
$$

(iv) L'intersection du spectre essentiel de $\tilde{H}_{W}$ et de l'axe réel est incluse dans :

$$
\tau(H)+\left[-\left(\frac{\operatorname{Im}(k)}{1+\operatorname{Re}(\theta)}\right)^{2},\left(\frac{\operatorname{Im}(k)}{\operatorname{Im}(\theta)}\right)^{2}\right]
$$

(v) Pour toutes fonctions $\psi, \phi \in A$ la fonction $g_{\psi, \phi}(z)=\left(\psi,(z-H)^{-1} \phi\right)_{L^{2}(X)}$ s'étend méromorphiquement de $\{z \in \mathbb{C} \mid \operatorname{Im}(z)>0\}$ à $\mathbb{C} \backslash S$ et l'on a :

$$
\sigma_{d}\left(\tilde{H}_{W}\right)=\cup_{\psi, \phi \in A}\left\{\text { pôles de } g_{\psi, \phi}(z)\right\}
$$

Explicitons ces résultats :

Remarquons tout d'abord que ce théorème englobe à la fois les cas des dilatations analytiques $(k=0$ et $\chi=1)$, des boost-dilatations $(\chi=1)$, et des distorsions analytiques $(k=0)$.

Pour que ces résultats soient utilisables dans la pratique il convient de localiser le spectre essentiel. Cela est fait grâce au point (iii) du théorème, qui 
montre que le spectre essentiel de l'opérateur $\tilde{H}_{W}$ est constitué d'un ensemble de paraboloïdes d'axes parallèles à la droite $\operatorname{Im}(z)=-2 \operatorname{Im}(\theta) \operatorname{Re}(z)$, et d'ouverture proportionnelle à $\operatorname{Im}(k)$. En particulier lorsque $k=0$, cela devient un ensemble de demi-droites, et l'on retrouve dans le cas $\chi=1$ les résultats bien connus sur les dilatations analytiques et le problème à $\mathrm{N}$ corps.

Le point (iv), qui n'est qu'une conséquence directe du (iii) mérite tout de même une attention particulière. Lorsque l'on fait tendre $\operatorname{Im}(k)$ et $\operatorname{Im}(\theta)$ vers zéro les paraboloides s'aplatissent et prennent une orientation plus proche de l'axe réel. Si en plus on maintient constant le rapport $\frac{\operatorname{Im}(k)}{\operatorname{Im}(\theta)}$ et que l'on s'intéresse à l'intersection du spectre essentiel de $\tilde{H}_{W}$ et de l'axe réel on constate que les deux effets se compensent, et que cette intersection tend vers l'ensemble :

$$
\cup_{x \in \tau(H)}\left\{x+\left[0,\left(\frac{\operatorname{Im}(k)}{\operatorname{Im}(\theta)}\right)^{2}\right]\right\}
$$

Ainsi on voit qu'un point $\lambda$ de l'axe réel n'est pas dans le spectre essentiel si $\operatorname{Im}(k)$ est assez petit et si $\left|\frac{\operatorname{Im}(k)}{\operatorname{Im}(\theta)}\right|<\sqrt{d(\lambda)}$ où $d(\lambda)$ est la distance entre $\lambda$ et le premier seuil qui lui est inférieur. Notons au passage que cette dernière condition est exactement celle que l'on obtient pour que l'opérateur $\frac{1}{2}\left(x . D_{x}+D_{x} . x\right)-\frac{\operatorname{Im}(k)}{\operatorname{Im}(\theta)}\langle x\rangle$ soit conjugué au Hamiltonien, au point $\lambda$, au sens de Mourre [Sk].

Pour effectuer la démonstration du théorème nous avons besoin de quelques résultats préliminaires.

Lemme A.4 - Pour toute fonction $\chi$ vérifiant les conditions (A.1) nous avons :

(i) Pour tout $\phi \in A$ l'application :

$$
\begin{cases}(\theta, k) & \rightarrow \tilde{W}(\theta, k) \phi \\ \{|\theta|<\sqrt{2}\} \times c & \rightarrow L^{2}(X)\end{cases}
$$

est analytique.

(ii) Pour chaque couple $(\theta, k) \in\{|\theta|<\sqrt{2}\} \times \mathbb{C}$ l'ensemble $\tilde{W}(\theta, k) A$ est dense dans $L^{2}(X)$.

On trouvera la démonstration de ce lemme dans l'article de Hunziker [Hu, Théorème 3]. 
Introduisons la partition de l'unité construite dans [Gr], $\left(j_{a}\right)_{a \in \mathcal{A}}$, indexée sur les l'ensemble des décompositions, qui a les propriétés suivantes :

1) $\forall a \in \mathcal{A}, j_{a} \in C^{\infty}\left(X, \mathbb{R}^{+}\right)$et les dérivées de $j_{a}$ sont bornées.

2) $\sum_{a \in \mathcal{A}} j_{a}(x)=1$

3) $\exists C_{0}, C_{1}$ telles que sur le support de $j_{a}$ :

$$
\left|x^{a}\right| \leq C_{0} \quad \text { et } \quad\left|x^{b}\right| \geq C_{1} \quad \forall b \not \subset a
$$

Définitions A.5 - Pour toute décomposition a et tout $\delta<1$ on pose :

$$
\begin{gathered}
q_{a}(x)=j_{a}\left(\frac{x}{\langle x\rangle^{\delta}}\right) \\
H_{a W}=\frac{1}{(1+\theta)^{2}}\left(D_{x_{a}}+k \frac{x_{a}}{\left\langle x_{a}\right\rangle}\right)^{2}+H_{\theta}^{a} \\
R_{a}(z)=q_{a}\left(H_{a W}-z\right)^{-1} \\
Q_{a}(z)=q_{a}\left(H_{a W}-z\right)^{-1}\left(H_{a W}-H_{W}\right)
\end{gathered}
$$

où l'on a noté $H_{W}$ l'opérateur $\tilde{H}_{W}$ pris dans le cas particulier ou $\chi=1$.

Lemme A.6 - (i) Pour tout complexe $k$ le spectre essentiel de l'opérateur $\left(D_{x}+k \frac{x}{\langle x\rangle}\right)^{2}$, opérant sur $X=\mathbb{R}^{p}$, est inclus dans le paraboloide $P(k)$ défini dans l'énoncé $d u$ Théorème A.3.

(ii) $\left(D_{x}+k \frac{x}{\langle x\rangle}\right)^{2}$ est un opérateur strictement $m$-sectoriel, au sens de Kato (Cf [Ka] V.3.10 pour la définition).

Démonstration du lemme A.6:

L'opérateur $\left(D_{x}+k \frac{x}{\langle x\rangle}\right)^{2}$ est l'opérateur pseudo-différentiel de symbole de Weyl $P(x, \xi)=\left(\xi+k \frac{x}{\langle x\rangle}\right)^{2}$. Notons que :

$$
P(x, \xi) \in S\left(\langle\xi\rangle^{2}, \frac{d x^{2}}{\langle x\rangle^{2}}+\frac{d \xi^{2}}{\langle\xi\rangle^{2}}\right)
$$

Pour $z \in \mathbb{C} \backslash P(k)$ on définit $P_{z}^{\prime}(x, \xi)=\frac{1}{P(x, \xi)-z}$ qui est dans la classe $S\left(\langle\xi\rangle^{-2}, \frac{d x^{2}}{\langle x\rangle^{2}}+\frac{d \xi^{2}}{\langle\xi\rangle^{2}}\right)$, car $|P(x, \xi)-z| \geq C\langle\xi\rangle^{2}$. On écrit :

$$
O p^{w}\left(\left(P\left(x, D_{x}\right)\right)-z\right) O p^{w}\left(P_{z}^{\prime}\left(x, D_{x}\right)\right)=1+O p^{w}\left(R_{z}\left(x, D_{x}\right)\right)
$$

où $R_{z}(x, \xi)$ est un symbole de classe $S\left(\frac{1}{\langle x\rangle\langle\xi\rangle}, \frac{d x^{2}}{\langle x\rangle^{2}}+\frac{d \xi^{2}}{\langle\xi\rangle^{2}}\right)$. L'opérateur associé est donc compact ( $\mathrm{Cf}[\mathrm{Ho}]$, tome III, théorème 18.6.6). On peut de plus, en estimant les semi-normes de $P_{z}^{\prime}(x, \xi)$, vérifier que pour tout secteur $\Gamma$ contenant $P(k)$ il existe une constante $C$ telle que :

$$
z \in \mathbb{C} \backslash \Gamma \Rightarrow\left\|O p^{w}\left(R_{z}\left(x, D_{x}\right)\right)\right\|_{L^{2}\left(\mathbb{R}^{p}\right)} \leq C \operatorname{dist}(z, \Gamma)^{-1}
$$


Ainsi pour $z \in \mathbb{R}$ et $-z>>1$ l'opérateur $\left(1+O p^{w}\left(R_{z}\left(x, D_{x}\right)\right)\right)$ est inversible et le théorème de Fredholm nous permet d'affirmer au vu de (A.3) que $\left(\left(D_{x}+k \frac{x}{\langle x\rangle}\right)^{2}-z\right)^{-1}$ est une fonction méromorphe sur $\mathbb{C} \backslash P(k)$.

Montrons maintenant (ii).

L'ensemble $P(k)$ étant un paraboloïde d'axe $\mathbb{R}$, pour tout $\theta>0$ on peut choisir, au vu de (A.4) un secteur :

$$
\Gamma_{z_{0}, \theta}=\left\{z \in \mathbb{C} \mid \operatorname{Arg}\left(z-z_{0}\right) \leq \theta\right\}
$$

tel que :

$$
z \in \mathbb{C} \backslash \Gamma_{z_{0}, \theta} \Rightarrow\left\|O p^{w}\left(R\left(x, D_{x}\right)\right)\right\|_{L^{2}\left(\mathbb{R}^{p}\right)} \leq \frac{1}{2}
$$

Ainsi d'après (A.3) pour $z \in \mathbb{R}, z<z_{0}$ :

$$
\left\|\left(O p^{w}\left(\left(P\left(x, D_{x}\right)\right)-z\right)\right)^{-1}\right\| \leq 2\left\|O p^{w}\left(P_{z}^{\prime}\left(x, D_{x}\right)\right)\right\| \leq 2 C_{0}\left|z-z_{0}\right|^{-1}
$$

et l'opérateur $\left(D_{x}+k \frac{x}{\langle x\rangle}\right)^{2}$ est m-accrétif au sens de Kato.

Pour monter qu'il est sectoriel nous remarquons que pour tout $u$ tel que $\left\langle D_{x}^{2} u\right\rangle \in L^{2}$ :

$$
\left|\left(\left(D_{x}+k \frac{x}{\langle x\rangle}\right)^{2} u, u\right)_{L^{2}(X)}-\left\|D_{x} u\right\|_{L^{2}(X)}^{2}\right| \leq|k|^{2}+|k|\left\|D_{x} u\right\|_{L^{2}(X)}
$$

et donc que le "numerical range" de $\left(D_{x}+k \frac{x}{\langle x\rangle}\right)^{2}$ est inclus dans le paraboloïde :

$$
\left\{z \in \mathbb{C} \mid \exists t \in \mathbb{R} \text { tel que }|z-t|^{2} \leq|k|^{2}+|k t|\right\}
$$

et par là même inclus dans un secteur d'angle aussi petit que l'on veut.

Lemme A.7 - (i) Les opérateurs $R_{a}(z)$ et $Q_{a}(z)$ sont analytiques sur $c \backslash S$.

(ii) $Q_{a}(z)$ est compact pour tout $z \in \mathbb{C} \backslash S$

(iii) $\left.\lim _{\operatorname{Re}(z) \rightarrow-\infty} \| Q_{a}(z)\right) \|=0$

Démonstration du lemme A.7:

Pour le premier point il nous suffit de montrer que $\sigma_{e}\left(H_{a W}\right) \subset S$. Pour cela écrivons :

$$
H_{a W}=\frac{1}{(1+\theta)^{2}}\left(D_{x_{a}}+k \frac{x_{a}}{\left\langle x_{a}\right\rangle}\right)^{2} \otimes I_{X^{a}}+I_{X_{a}} \otimes H_{\theta}^{a}
$$

Chacun des opérateurs $\frac{1}{(1+\theta)^{2}}\left(D_{x_{a}}+k \frac{x_{a}}{\left\langle x_{a}\right\rangle}\right)^{2}$ et $H_{\theta}^{a}$ étant strictement $m$ - sectoriel on a :

$$
\sigma\left(H_{a W}\right)=\frac{1}{(1+\theta)^{2}} \sigma\left(\left(D_{x_{a}}+k \frac{x_{a}}{\left\langle x_{a}\right\rangle}\right)^{2}\right)+\sigma\left(H_{\theta}^{a}\right)
$$


Le spectre de $\left(H_{\theta}^{a}\right)$ est bien connu et s'écrit :

$$
\sigma\left(H_{\theta}^{a}\right)=\sigma_{d}\left(H_{\theta}^{a}\right) \underset{b \subset a, b \neq a}{\cup}\left\{\sigma_{d}\left(H_{\theta}^{b}\right)+\frac{1}{(1+\theta)^{2}} \mathbb{R}^{+}\right\}
$$

D'autre part le spectre essentiel de $\left(D_{x_{a}}+k \frac{x_{a}}{\left\langle x_{a}\right\rangle}\right)^{2}$ est inclus dans le paraboloïde $P(k)$ d'après le lemme A.6. Comme $P(k)+\mathbb{R}^{+}=P(k)$ on obtient effectivement $\sigma_{e}\left(H_{a W}\right) \subset S$.

Pour montrer que $Q_{a}(z)$ est compact on calcule :

$$
\begin{aligned}
(1+\theta)^{2}\left(H_{a W}-H_{W}\right)= & k\left(\frac{1}{\left\langle x_{a}\right\rangle}-\frac{1}{\langle x\rangle}\right) x_{a} \cdot D_{x_{a}}-k \frac{x^{a}}{\langle x\rangle} D_{x^{a}}+\operatorname{sym} \\
& +k^{2}\left|x_{a}\right|^{2}\left(\frac{1}{\left\langle x_{a}\right\rangle^{2}}-\frac{1}{\langle x\rangle^{2}}\right)+k^{2} \frac{\left|x^{a}\right|^{2}}{\langle x\rangle^{2}} \\
& +(1+\theta)^{2} I_{a}((1+\theta) x)
\end{aligned}
$$

d'où l'on en déduit que $\left(H_{a W}-z\right)^{-1} q_{a}\left(H_{a W}-H_{W}\right)$ est compact. Nous écrivons alors :

$$
\begin{aligned}
Q_{a}(z)= & \left(H_{a W}-z\right)^{-1} q_{a}\left(H_{a W}-H_{W}\right) \\
& +\left(\left(H_{a W}-z\right)^{-1}\left[H_{a W}, q_{a}\right]\right) \times\left(\left(H_{a W}-z\right)^{-1}\left(H_{a W}-H_{W}\right)\right)
\end{aligned}
$$

ce qui ne fait apparaitre qu'une somme d'opérateurs compacts.

Enfin pour montrer le point (iii) nous écrivons pour $\lambda_{0}>1$ :

$$
\begin{aligned}
\left(H_{a W}-z\right)^{-1}\left(H_{a W}-H_{W}\right) & =\left(\left(H_{a W}-z\right)^{-1}\left(H_{a W}+\lambda_{0}\right)\right) \\
\times & \left(\left(H_{a W}+\lambda_{0}\right)^{-1}\left(H_{a W}-H_{W}\right)\right)
\end{aligned}
$$

et il apparait clairement que $\lim _{R e(z) \rightarrow-\infty}\left\|\left(H_{a W}-z\right)^{-1}\left(H_{a W}-H_{W}\right)\right\|=0$.

Démonstration du théorème A.3 :

Pour montrer $(i)$ nous écrivons :

$$
\tilde{H}_{W}=\tilde{W}(\theta, k) D_{x}^{2} \tilde{W}(\theta, k)^{-1}+V((1+\theta \chi(x)) x)
$$

Le terme $V((1+\theta \chi(x)) x)$ a, d'après les hypothèses $(H)$, une extension holomorphe en $\theta$ et est borné. Quant à l'opérateur $\tilde{W}(\theta, k) D_{x}^{2} \tilde{W}(\theta, k)^{-1}$, c'est un opérateur différentiel du second ordre, holomorphe en $\theta$, de symbole principal :

$$
\tilde{p}(x, \xi)=\left(A(x)\left(\xi+k \frac{x}{\langle x\rangle}\right),\left(\xi+k \frac{x}{\langle x\rangle}\right)\right)
$$

où $A(x)=B(x)^{-1}, B(x)={ }^{t} J J$ et $J=1+\theta \nabla \chi$. (Cf [Hu]).

Pour $\theta$ assez petit on a :

$$
|\tilde{p}(x, \xi)| \geq C|\xi|^{2}
$$


de telle sorte que le domaine de $\tilde{W}(\theta, k) D_{x}^{2} \tilde{W}(\theta, k)^{-1}$, et par conséquent celui de $\tilde{H}_{W}$, est égal à celui de $D_{x}^{2}$, indépendamment de $\theta$ et $k$.

Pour démontrer les points $(i i)$ à $(v)$ nous reprenons le schéma classique de la démonstration que l'on trouve par exemple dans [Ba-Co] et [Hu].

La principale difficulté consiste à localiser le spectre essentiel de $\tilde{H}_{W}$. Pour cela nous procéderons en deux temps : nous montrerons tout d'abord que le point $(i i i)$ du théorème tient dans le cas $\chi=1$, puis nous en déduirons le cas général.

Dans le cas $\chi=1$ nous calculons :

$$
\begin{aligned}
\left(H_{W}-z\right)^{-1}= & \sum_{a \in \mathcal{A}} q_{a}\left(H_{W}-z\right)^{-1} \\
= & \left(\sum_{a \in \mathcal{A}} Q_{a}(z)\right)\left(H_{W}-z\right)^{-1} \\
& +\sum_{a \in \mathcal{A}} R_{a}(z)
\end{aligned}
$$

et le lemme A.7 nous permet d'affirmer que $\left(H_{W}-z\right)^{-1}$ est méromorphe sur $c \backslash S$, et ainsi que $\sigma_{e}\left(H_{W}\right) \subset S$. Intéressons-nous maintenant au cas $\chi \neq 1$. Pour éviter toute confusion nous utiliserons la notation $H_{W}$ pour le cas $\chi=1$ et $\tilde{H}_{W}$ pour le cas $\chi \neq 1$.

Posons alors pour tout $z \in \rho\left(H_{W}\right) \cap \rho\left(\tilde{H}_{W}\right) \backslash S$ :

$$
R_{W}(z)=\left(H_{W}-z\right)^{-1} ; \quad \tilde{R}_{W}(z)=\left(\tilde{H}_{W}-z\right)^{-1} \text { et } K_{W}=\tilde{R}_{W}(z)-R_{W}(z)
$$

Nous avons :

$$
\begin{aligned}
K_{W}= & \tilde{R}_{W}(z)\left(H_{W}-\tilde{H}_{W}\right)-R_{W}(z) \\
= & \tilde{R}_{W}(z)(V((1+\theta) x)-V((1+\theta \chi(x)) x)) R_{W}(z) \\
& +\tilde{R}_{W}(z)\left(W(\theta, k) D_{x}^{2} W(\theta, k)^{-1}-\tilde{W}(\theta, k) D_{x}^{2} \tilde{W}(\theta, k)^{-1}\right) R_{W}(z)
\end{aligned}
$$

Le premier terme de cette expression est compact car $V((1+\theta) x)-V((1+\theta \chi(x)) x)$ est un potentiel à support compact. Quant au deuxième terme il est aussi compact car l'opérateur $W(\theta, k) D_{x}^{2} W(\theta, k)^{-1} \quad-\quad \tilde{W}(\theta, k) D_{x}^{2} \tilde{W}(\theta, k)^{-1} \quad$ est un opérateur différentiel de second ordre de symbole principal $\left((A(x)-1)\left(\xi+k \frac{x}{\langle x\rangle}\right),\left(\xi+k \frac{x}{\langle x\rangle}\right)\right)$, et $A(x)-1$ est à support compact. Nous constatons ainsi que $K_{W}$ est toujours compact. D'après le "Strong Spectral Mapping Theorem" [R-S] :

$$
\sigma_{e}\left(R_{W}(z)\right) \subset\left\{\frac{1}{t-z} ; t \in \sigma_{e}\left(H_{W}\right)\right\} \cup\{0\}
$$

et ainsi :

$$
\sigma_{e}\left(R_{W}(z)\right) \subset G_{z} \quad \text { avec } \quad G_{z}:=\left\{\frac{1}{t-z} ; t \in S\right\}
$$


Pour $z \notin S$ l'ensemble $G_{z}$ est borné et son complementaire, $c \backslash G_{z}$ est connexe. L'opérateur :

$$
B_{W}(t)=K_{W}\left(R_{W}(z)-t\right)^{-1}
$$

est compact, méromorphe en $t$ sur $\mathrm{c} \backslash G_{z}$. Pour $\left|t_{0}\right|$ assez grand $\left(\tilde{R}_{W}(z)-t_{0}\right)^{-1}$ existe et $\left(\left(R_{W}(z)-t_{0}\right)\left(\tilde{R}_{W}(z)-t_{0}\right)^{-1}\right)\left(1+B_{W}\left(t_{0}\right)\right)=1$. Ainsi d'après le théorème de Fredholm nous savons que $\left(1+B_{W}(t)\right)$ est méromorphe sur c $\backslash G_{z}$, et par là même que :

$$
\sigma_{e}\left(\tilde{R}_{W}(z)\right) \subset G_{z}
$$

Utilisant à nouveau le "Strong Spectral Mapping Theorem" il vient :

$$
\left\{\frac{1}{t-z} ; t \in \sigma_{e}\left(\tilde{H}_{W}\right)\right\} \subset G_{z}=\left\{\frac{1}{t-z} ; t \in S\right\}
$$

et pour finir $\sigma_{e}\left(\tilde{H}_{W}\right) \subset S$, ce qui achève la démonstration de $(i i i)$, et de $(i v)$, qui en est une conséquence directe.

Démontrons $(v)$.

Pour $\theta$ et $k$ réels l'opérateur $\tilde{W}(\theta, k)$ est unitaire de telle sorte que nous avons encore :

$$
g_{\psi, \phi}(z)=\left(\tilde{W}(\bar{\theta}, \bar{k}) \psi,\left(\tilde{H}_{W}-z\right)^{-1} \tilde{W}(\theta, k) \phi\right)_{L^{2}(X)}
$$

D'apres $(i)$ et le lemme A.4 cette expression, à $z$ fixé, se prolonge holomorphiquement en $(\theta, k)$ sur un domaine $\{|\theta| \leq \epsilon\} \times\{k \in \mathbb{C}\}$. De plus étant constante sur le réel, elle est constante sur tout le domaine d'analyticité et l'expression (A.5) reste valable pour $\theta$ et $k$ complexes.

Fixons maintenant $\theta$ et $k$, complexes, les points $(i)$ et (iii) nous permettent d'affirmer que (A.5) s'étend méromorphiquement en $z$ sur $C \backslash S$, et que ses pôles sont des valeurs propres de $\tilde{H}_{W}$.

Il ne nous reste donc plus qu'à montrer que :

$$
\sigma_{d}\left(\tilde{H}_{W}\right) \subset \cup_{\psi, \phi \in A}\left\{\text { pôles de } g_{\psi, \phi}(z)\right\}
$$

Pour cela prenons un élément $\lambda$ de $\sigma_{d}\left(\tilde{H}_{W}\right)$ et notons $Q_{0}$ le résidu d'ordre 1 de $\left(\tilde{H}_{W}-z\right)^{-1}$ au pole $z=\lambda$. L'ensemble $\tilde{W}(\theta, k) A$ étant dense dans $L^{2}(X)$, d'après le lemme A.4, nous pouvons choisir $\psi$ et $\phi$ dans $A$ tels que :

$$
\left(\tilde{W}(\bar{\theta}, \bar{k}) \psi, Q_{0} \tilde{W}(\theta, k) \phi\right) \neq 0
$$

Le point $z=\lambda$ est alors un pôle de $g_{\psi, \phi}(z)$ et nous avons montré (A.6).

Enfin remarquons que le point (ii) du théorème est une conséquence de (A.2), étant donné que les fonctions $g_{\psi, \phi}(z)$ sont définies indépendamment de la fonction $\chi$. 


\section{Bibliographie}

[Ba1] E. Balslev : Analytic Scattering Theory for Two-Body Scrödinger operators, J. Fun. Anal. 29, 375-395 (1978)

[Ba2] E. Balslev : Local Spectral Deformation Techniques for Scrödinger operators, J. Fun. Anal. 58, 79-105 (1984)

[Ba3] E. Balslev : Analytic Scattering Theory for Many-Body Systems Below the Smallest three-Body Threshold, Com. Math. Phys. 77, 173-210 (1980)

[Ba-Co] E. Baslev, J.M. Combes : Spectral properties of many-body Schrödinger operators with dilatation-analytic interactions, Com. Math. Phys. 22, 280-294 (1971).

[Bg] T. Bang Om Quasi-analytische Funktionen, Thèse, Univ. de Copenhague (1946).

[Bo1] A. Bommier : Propriétes de la matrice de diffusion 2amas -2 amas, pour les problèmes à $N$ corps à longue portée, Annales de l'I.H.P. vol $59 \mathrm{n}^{0}$ 3, 237-267 (1993).

[Bo2] A. Bommier : Régularité et prolongement méromorphe de la matrice de diffusion pour les problèmes à $N$ corps à longue portée, Thèse de doctorat de l'Ecole Polytechnique. Non publiée (1993).

[C-Z] L. Cattabriga L. Zanghirati : Fourier Integral Operators of Infinite Order on Gevrey Spaces. Applications to the Cauchy Problem for Hyperbolic Operators, dans Advances in Microlocal Analysis, série C, vol 168, Nato ASI Series (1985).

[Co-T] J.M. Combes L. Thomas : Asymptotic Behaviour of Eigenfunctions for Multiparticle Schrödinger Operators, Com. Math. Phys. 34, 251-270 (1973). 
[F-H] R.Froese-I.Herbst : Exponential Bounds and Absence of Positive eigenvalues for $N$-Body Schrödinger Operators, Com. Math. Phys. 87, 429-447 (1982).

[G-M] C.Gérard A.Martinez : Prolongement méromorphe de la matrice de scattering pour des problèmes à deux corps à longue portée, Ann. I.H.P. vol $51 n^{\circ}$ 1, 81-110 (1989).

[Gr] G.M. Graf : Asymptotic completness for $N$-body Short-Range Quantum Systems : a new proof, Com. Math. Phys. 132, 73-101 (1990).

[Ho] L.Hörmander : The analysis of partial Differential Operators, vol 3, Springer-Verlag, New-York, 1985.

[Hu] W. Hunziker : Distortion analyticity and molecular resonance curves, Ann.I.H.P. Vol $45 n^{\circ}$ 4, 339-358 (1986).

[I-K] H.Isozaki-H.Kitada : Scattering Matrices for Two-Body Schrödinger Operators, Scientific Papers of the College of Arts and Sciences, Tokyo University, 35, 81-107 (1985).

[Ka] T.Kato : Perturbation theory for linear operators, Berlin. Heidelberg. New York : Springer-Verlag (1966).

[L] P. Laubin : Analyse microlocale des singularités analytiques, Bull. Soc. Roy. Sci. Liège, vol 52, 103-212 (1983).

[R-S] M.Reed-B.Simon : Methods of Modern Mathematical Physics, vol 1-4, (Academic,London 1975, 1978, 1979 et 1980).

[Sh-Th] N.Shenk-D.Toe : Resonant states and poles of the scattering matrix for perturbations of $-\Delta$, J. Math. Anal. Appl. vol 54, 316-349 (1976)

[Sk] E.Skibsted : Smoothness of $N$-body scattering amplitudes , Rev.Math.Phys. vol 4, $\mathrm{n}^{\circ} 4,619-658$ (1992).

[W] X.P.Wang: Time-delay operators in semiclassical limit. II. Shortrange potentials, Trans.Am.Math.Soc. 322-1, 395-414 (1990).

Remerciements : L'étude présentée ici a été effectuée dans le cadre de mon travail de thèse [Bo2], dirigé par Christian Gérard, que je remercie pour ses nombreux conseils et idées. 